MATHEMATICS OF COMPUTATION

Volume 82, Number 282, April 2013, Pages 1139-1179

S 0025-5718(2012)02633-0

Article electronically published on November 20, 2012

\title{
ECM USING EDWARDS CURVES
}

\author{
DANIEL J. BERNSTEIN, PETER BIRKNER, TANJA LANGE, AND CHRISTIANE PETERS
}

\begin{abstract}
This paper introduces EECM-MPFQ, a fast implementation of the elliptic-curve method of factoring integers. EECM-MPFQ uses fewer modular multiplications than the well-known GMP-ECM software, takes less time than GMP-ECM, and finds more primes than GMP-ECM. The main improvements above the modular-arithmetic level are as follows: (1) use Edwards curves instead of Montgomery curves; (2) use extended Edwards coordinates; (3) use signed-sliding-window addition-subtraction chains; (4) batch primes to increase the window size; (5) choose curves with small parameters and base points; (6) choose curves with large torsion.
\end{abstract}

\section{INTRODUCTION}

Factorization of integers is one of the most studied problems in algorithmic number theory and cryptology. One of the best general factorization methods available is the Elliptic-Curve Method (ECM), introduced in the 1987 paper 33 . by Hendrik W. Lenstra, Jr. ECM plays an important role in factoring the "random" integers of interest to number theorists: it is not as fast as trial division and Pollard's rho method for finding tiny prime factors, but it is the method of choice for finding medium-size prime factors. ECM also plays an important role in factoring the "hard" integers of interest to cryptologists: those integers are attacked by sieving methods, which use ECM to find medium-size prime factors of auxiliary integers. ECM can also be used directly to find "large" prime factors; the current record (see [46]) is a 241-bit factor of the 1181-bit number $2^{1181}-1$.

Implementations of ECM are available in most computer-algebra packages and have been the subject of many papers. The state-of-the-art implementation is GMP-ECM, described in detail in the paper [4] by Zimmermann and Dodson.

We have built a new ECM implementation, "EECM-MPFQ", that uses fewer modular multiplications than GMP-ECM, takes less time than GMP-ECM, and finds more primes than GMP-ECM. Our first prototype of EECM-MPFQ was "GMP-EECM", a program that added various improvements to GMP-ECM; we thank Zimmermann et al. for making their software freely available!

Received by the editor December 29, 2009 and, in revised form, October 8, 2011.

2010 Mathematics Subject Classification. Primary 11Y05; Secondary 11G05.

Key words and phrases. Factorization, ECM, elliptic-curve method, curve selection, Edwards coordinates, extended Edwards coordinates.

Permanent ID of this document: cb39208064693232e4751ec8f3494c43. This work was supported in part by the European Commission through the ICT Programme under Contract ICT2007-216676 ECRYPT-II, and in part by the National Science Foundation under grant ITR0716498. This work was carried out while the fourth author was with Technische Universiteit Eindhoven; in part while the first author was visiting Technische Universiteit Eindhoven; and in part while the authors were visiting INRIA Nancy. 
In this paper we present the background and speed results for EECM-MPFQ. To simplify verification and reuse of our results we have published the EECMMPFQ software at http://eecm.cr.yp.to and placed it into the public domain. We are continuing to update http://eecm.cr.yp.to with new information about EECM: for example, the followup paper [11] reports a very fast parallel implementation of EECM using graphics cards as a computational platform.

1.1. Representations of elliptic curves. Elliptic curves can be expressed in many forms, and elliptic-curve computations can be carried out in many ways. Two fast options reigned supreme for twenty years of elliptic-curve factoring, ellipticcurve primality proving, and (in large characteristic) elliptic-curve cryptography:

- Short Weierstrass curves $y^{2}=x^{3}+a_{4} x+a_{6}$, with Jacobian coordinates $(X: Y: Z)$ representing $\left(X / Z^{2}, Y / Z^{3}\right)$, were the representation of choice for most computations.

- Montgomery curves $B y^{2}=x^{3}+A x^{2}+x$, with Montgomery coordinates $(X: Z)$ representing two points $(X / Z, \pm \cdots)$, were the representation of choice for single-scalar multiplication, and in particular for stage 1 of ECM.

The picture changed in 2007 with the advent of Edwards curves. A sequence of papers [13, 10], 14, 15], and 29] showed that, for cryptographic applications, Edwards curves involve significantly fewer multiplications than short Weierstrass curves in Jacobian coordinates, and - for sufficiently large scalar multiplications fewer multiplications than Montgomery curves in Montgomery coordinates. Note that larger scalars benefit from larger windows, reducing the number of additions per bit for Edwards coordinates but not for Montgomery coordinates.

1.2. Contributions of this paper. In this paper we analyze the impact of Edwards curves on ECM, not just in multiplication counts but also in real-world software speeds.

Section 2 discusses the group law on Edwards curves and twisted Edwards curves, and reviews various coordinate systems for Edwards curves. Our prototype GMPEECM used twisted inverted Edwards coordinates, and EECM-MPFQ uses extended Edwards coordinates. Section 3 analyzes points of small order on Edwards curves. Sections 4 and 5 discuss the use of Edwards curves inside ECM. Our announcement of GMP-EECM in January 2008 marked the first time that Edwards curves had been demonstrated to achieve software speed records.

A large portion of this paper is devoted to explaining which curves we use in EECM-MPFQ. Curves having 12 or 16 torsion points over $\mathbf{Q}$ are guaranteed to have 12 or 16 as divisors of their group orders modulo primes (of good reduction), improving the smoothness chance of the group orders and thus improving the success chance of ECM. We show how to use analogous improvements for Edwards curves; even better, we find new curves with large torsion group, small curve parameters, and small nontorsion points.

Section 6 explains how to construct Edwards curves having torsion group $\mathbf{Z} / 12 \mathbf{Z}$ or $\mathbf{Z} / 2 \mathbf{Z} \times \mathbf{Z} / 8 \mathbf{Z}$ over $\mathbf{Q}$; the symmetry of Edwards curves simplifies the constructions. Section 6 also shows that twisted Edwards curves cannot have torsion group $\mathbf{Z} / 2 \mathbf{Z} \times \mathbf{Z} / 6 \mathbf{Z}$ or $\mathbf{Z} / 10 \mathbf{Z}$ over $\mathbf{Q}$, and that twisted Edwards curves with torsion group $\mathbf{Z} / 12 \mathbf{Z}$ or $\mathbf{Z} / 2 \mathbf{Z} \times \mathbf{Z} / 8 \mathbf{Z}$ over $\mathbf{Q}$ cannot have curve parameter $a=-1$. Section $\mathbf{7}$. adapting a construction of Atkin and Morain from [3] to the Edwards context, explains how to construct an infinite family of Edwards curves having torsion group 
$\mathbf{Z} / 2 \mathbf{Z} \times \mathbf{Z} / 8 \mathbf{Z}$ and (as required for ECM) an explicit nontorsion point; and similarly adapts a construction of Montgomery for $\mathbf{Z} / 12 \mathbf{Z}$. Section 8 describes how we found better choices of Edwards curves to use in EECM-MPFQ; each of these curves has torsion group $\mathbf{Z} / 12 \mathbf{Z}$ or $\mathbf{Z} / 2 \mathbf{Z} \times \mathbf{Z} / 8 \mathbf{Z}$, an explicit nontorsion point, and small (i.e., fast) parameters.

Section 9 reports measurements of ECM success probabilities, demonstrating the importance of a large torsion group. Section 10 reports the overall effectiveness of EECM-MPFQ when parameters are chosen sensibly; for example, it shows that one curve finds $13.414 \%$ of all 30 -bit primes in just 3065 modular multiplications.

See 9 for followup work exploring the price-performance ratio of the $a=-1$ case of twisted Edwards curves in ECM.

\section{EDWARDS CURVES}

This section reviews twisted Edwards curves, and Edwards curves as a special case; the set of points on a twisted Edwards curve in affine, projective, inverted, extended, and completed forms; the Edwards addition law and a dual addition law, together turning the completed twisted Edwards curve into a group; and the speeds of addition and doubling in various representations.

For a collection of explicit formulas and operation counts for elliptic curves in various representations we refer to the Explicit-Formulas Database [12.

2.1. Edwards curves and twisted Edwards curves. Let $k$ be a field in which $2 \neq 0$, and let $a, d$ be distinct nonzero elements of $k$. The twisted Edwards curve $\mathrm{E}_{\mathrm{E}, a, d}$ is given by

$$
\mathrm{E}_{\mathrm{E}, a, d}: a x^{2}+y^{2}=1+d x^{2} y^{2} .
$$

An Edwards curve is a twisted Edwards curve in which $a=1$; i.e., a curve of the form $x^{2}+y^{2}=1+d x^{2} y^{2}$ where $d \in k \backslash\{0,1\}$.

If $a \bar{d}=\bar{a} d$, then the two curves $\mathrm{E}_{\mathrm{E}, a, d}$ and $\mathrm{E}_{\mathrm{E}, \bar{a}, \bar{d}}$ are isomorphic over $k(\sqrt{a / \bar{a}})$ and therefore are quadratic twists over $k$. An isomorphism is given by $(x, y) \mapsto$ $(\bar{x}, \bar{y})=(\sqrt{a / \bar{a}} x, y)$. In particular, the twisted Edwards curve $\mathrm{E}_{\mathrm{E}, a, d}$ is a quadratic twist of the Edwards curve $\mathrm{E}_{\mathrm{E}, 1, d / a}$.

Five slightly different ways to build a set of points from an Edwards curve, or more generally a twisted Edwards curve, have appeared in the literature. The simplest is the set of affine points $\left\{(x, y) \in \mathbf{A}^{2}: a x^{2}+y^{2}=1+d x^{2} y^{2}\right\}$. Four others, with various theoretical and computational advantages, are the projective, inverted, extended, and completed sets discussed below.

2.2. The Edwards addition law. The Edwards addition law on $\mathrm{E}_{\mathrm{E}, a, d}$ is given in affine coordinates by

$$
\left(x_{1}, y_{1}\right),\left(x_{2}, y_{2}\right) \mapsto\left(\frac{x_{1} y_{2}+y_{1} x_{2}}{1+d x_{1} x_{2} y_{1} y_{2}}, \frac{y_{1} y_{2}-a x_{1} x_{2}}{1-d x_{1} x_{2} y_{1} y_{2}}\right) .
$$

By inserting appropriate denominators one obtains the Edwards addition law in projective coordinates, inverted coordinates, extended coordinates, and completed coordinates.

The Edwards addition law is strongly unified; i.e., the same formulas can also be used for generic doublings. The point $(0,1)$ is the neutral element of the addition law. The negative of a point $\left(x_{1}, y_{1}\right)$ is $\left(-x_{1}, y_{1}\right)$. 
The Edwards addition law for $\mathrm{E}_{\mathrm{E}, a, d}$ was studied by Bernstein, Birkner, Joye, Lange, and Peters in [8, generalizing from the case $a=1$ studied by Bernstein and Lange in [13, generalizing from the case $a=1, d=c^{4}$ studied by Edwards in 25], generalizing from the case $a=1, d=-1$ studied by Euler and Gauss.

Edwards actually used the form $x^{2}+y^{2}=c^{2}\left(1+x^{2} y^{2}\right)$. Edwards showed that every elliptic curve over $\mathbf{Q}$ can be written in this normal form over an extension of Q. Replacing $(x, y)$ with $(c x, c y)$ produces the curve $\mathrm{E}_{\mathrm{E}, 1, c^{4}}$; this scaling turns out to save time in computations. The further generalizations to $\mathrm{E}_{\mathrm{E}, 1, d}$ and to $\mathrm{E}_{\mathrm{E}, a, d}$ allow more curves over $\mathbf{Q}$ to be handled at similar speeds.

2.3. The dual addition law. Hisil, Wong, Carter, and Dawson in [29] introduced the addition law

$$
\left(x_{1}, y_{1}\right),\left(x_{2}, y_{2}\right) \mapsto\left(\frac{x_{1} y_{1}+x_{2} y_{2}}{y_{1} y_{2}+a x_{1} x_{2}}, \frac{x_{1} y_{1}-x_{2} y_{2}}{x_{1} y_{2}-y_{1} x_{2}}\right)
$$

on $\mathrm{E}_{\mathrm{E}, a, d}$. This dual addition law produces the same output as the Edwards addition law when both are defined, but the exceptional cases are different. In particular, the dual addition law never works for doublings: if $\left(x_{1}, y_{1}\right)=\left(x_{2}, y_{2}\right)$, then the second output coordinate $\left(x_{1} y_{1}-x_{2} y_{2}\right) /\left(x_{1} y_{2}-y_{1} x_{2}\right)$ is $0 / 0$. The dual addition law nevertheless has some important advantages, as discussed below.

2.4. Projective points. The projective twisted Edwards curve is

$$
\left\{(X: Y: Z) \in \mathbf{P}^{2}: a X^{2} Z^{2}+Y^{2} Z^{2}=Z^{4}+d X^{2} Y^{2}\right\} .
$$

The projective points are the affine points $\left(x_{1}, y_{1}\right)$, embedded as usual into $\mathbf{P}^{2}$ by $\left(x_{1}, y_{1}\right) \mapsto\left(x_{1}: y_{1}: 1\right)$, and two extra singular points at infinity, namely $(0: 1: 0)$ and $(1: 0: 0)$.

Fast projective addition and doubling formulas, starting from the Edwards addition law and eliminating multiplications in various ways, were introduced for Edwards curves in [13] and were generalized to twisted Edwards curves in [8]. Adding a generic pair of points uses just $10 \mathbf{M}+1 \mathbf{S}+1 \mathbf{A}+1 \mathbf{D}$ : i.e., 10 field multiplications, 1 field squaring, 1 multiplication by $a$, and 1 multiplication by $d$. Doubling takes just $3 \mathbf{M}+4 \mathbf{S}+1 \mathbf{A}$ with the following formulas:

$$
\begin{aligned}
B & =\left(X_{1}+Y_{1}\right)^{2} ; C=X_{1}^{2} ; D=Y_{1}^{2} ; E=a C ; F=E+D ; H=Z_{1}^{2} ; \\
J & =F-2 H ; X_{3}=(B-C-D) \cdot J ; Y_{3}=F \cdot(E-D) ; Z_{3}=F \cdot J .
\end{aligned}
$$

These doubling formulas are used in EECM-MPFQ.

2.5. Inverted points. The inverted twisted Edwards curve is

$$
\left\{(X: Y: Z) \in \mathbf{P}^{2}: a Y^{2} Z^{2}+X^{2} Z^{2}=X^{2} Y^{2}+d Z^{4}\right\} .
$$

The inverted points are the affine points $\left(x_{1}, y_{1}\right)$ other than $(0, \pm 1)$ and $( \pm 1,0)$, embedded into $\mathbf{P}^{2}$ by $\left(x_{1}, y_{1}\right) \mapsto\left(1 / x_{1}: 1 / y_{1}: 1\right)$; two extra points if $d$ is a square, namely $( \pm \sqrt{d}: 0: 1)$; two extra points if $d / a$ is a square, namely $(0: \pm \sqrt{d / a}: 1)$; and two singular points at infinity, namely $(0: 1: 0)$ and $(1: 0: 0)$. Note that a generic inverted point $\left(X_{1}: Y_{1}: Z_{1}\right)$ corresponds to the affine point $\left(Z_{1} / X_{1}, Z_{1} / Y_{1}\right)$.

Fast inverted addition and doubling formulas were introduced for Edwards curves in [14] and for twisted Edwards curves in [8]. Adding a generic pair of points costs only $9 \mathbf{M}+1 \mathbf{S}+1 \mathbf{A}+1 \mathbf{D}$, saving $1 \mathbf{M}$ compared to projective Edwards coordinates. A doubling costs $3 \mathbf{M}+4 \mathbf{S}+1 \mathbf{A}+1 \mathbf{D}$, losing $1 \mathbf{D}$ compared to projective Edwards coordinates. 
These formulas were used in the prototype GMP-EECM.

2.6. Extended points. The extended twisted Edwards curve is

$$
\left\{(X: Y: Z: T) \in \mathbf{P}^{3}: a X^{2}+Y^{2}=Z^{2}+d T^{2} \text { and } X Y=Z T\right\} .
$$

The extended points are the affine points $\left(x_{1}, y_{1}\right)$, embedded into $\mathbf{P}^{3}$ by $\left(x_{1}, y_{1}\right) \mapsto$ $\left(x_{1}: y_{1}: 1: x_{1} y_{1}\right)$; two extra points at infinity if $d$ is a square, namely $(0: \pm \sqrt{d}: 0$ : 1 ); and two extra points at infinity if $d / a$ is a square, namely $(1: 0: 0: \pm \sqrt{a / d})$.

Hisil, Wong, Carter, and Dawson in 29] introduced extended addition formulas costing only $9 \mathbf{M}+1 \mathbf{A}$ :

$$
\begin{aligned}
& A=X_{1} \cdot X_{2}, B=Y_{1} \cdot Y_{2}, C=Z_{1} \cdot T_{2}, D=T_{1} \cdot Z_{2}, \\
& E=D+C, F=\left(X_{1}-Y_{1}\right) \cdot\left(X_{2}+Y_{2}\right)+B-A, G=B+a A, \\
& H=D-C, X_{3}=E \cdot F, Y_{3}=G \cdot H, Z_{3}=F \cdot G, T_{3}=E \cdot H .
\end{aligned}
$$

These formulas save 1S by switching from inverted coordinates to extended coordinates, and an extra $1 \mathbf{D}$ by switching from the Edwards addition law to the dual addition law. These formulas are used in EECM-MPFQ. Our main concern in this paper is with the case $a=1$, where these formulas cost only $9 \mathbf{M}$. Hisil et al. also introduced addition formulas costing only $8 \mathbf{M}$ for the case $a=-1$; but we show in Section 6 that the case $a=-1$ sacrifices torsion.

A doubling in extended coordinates loses $1 \mathbf{M}$ for computing the extended output coordinate $T_{3}$. However, the doubling formulas make no use of the extended input coordinate $T_{1}$, so if the input is not used for anything else then the operation producing that input can skip the computation of $T_{1}$, saving $1 \mathrm{M}$.

Scalar multiplication can be carried out as a series of operations on an accumulator $P$ : doublings replace $P$ by $2 P$, and double-and-add operations replace $P$ by $2 P+Q$. If $P$ is in projective coordinates and the precomputed points $Q$ are in extended coordinates, then doubling costs $3 \mathbf{M}+4 \mathbf{S}+1 \mathbf{A}$ and double-and-add costs $(3 \mathbf{M}+4 \mathbf{S}+1 \mathbf{A})+(9 \mathbf{M}+1 \mathbf{A})$, with the $1 \mathbf{M}$ loss in doubling cancelled by the $1 \mathbf{M}$ savings in addition. This mixture of projective coordinates and extended coordinates was suggested in 29 and is used in EECM-MPFQ.

2.7. Completed points. The completed twisted Edwards curve is

$$
\overline{\mathrm{E}}_{\mathrm{E}, a, d}=\left\{((X: Z),(Y: T)) \in \mathbf{P}^{1} \times \mathbf{P}^{1}: a X^{2} T^{2}+Y^{2} Z^{2}=Z^{2} T^{2}+d X^{2} Y^{2}\right\} .
$$

The completed points are the affine points $\left(x_{1}, y_{1}\right)$, embedded as usual into $\mathbf{P}^{1} \times \mathbf{P}^{1}$ by $\left(x_{1}, y_{1}\right) \mapsto\left(\left(x_{1}: 1\right),\left(y_{1}: 1\right)\right)$; two extra points at infinity if $d$ is a square, namely $((1: \pm \sqrt{d}),(1: 0))$; and two extra points at infinity if $d / a$ is a square, namely $((1: 0),( \pm \sqrt{a / d}: 1))$. Starting in Section 6 we freely abbreviate $\left(\left(x_{1}: 1\right),\left(y_{1}: 1\right)\right)$ as $\left(x_{1}, y_{1}\right)$.

The completed curve maps isomorphically to the extended curve via the Segre embedding $((X: Z),(Y: T)) \mapsto(X T: Y Z: Z T: X Y)$ of $\mathbf{P}^{1} \times \mathbf{P}^{1}$ into $\mathbf{P}^{3}$. It maps onto the projective curve via $((X: Z),(Y: T)) \mapsto(X T: Y Z: Z T)$, but this map is not an isomorphism: it sends the two points $((1: \pm \sqrt{d}),(1: 0))$ to $(0: 1: 0)$, and sends the two points $((1: 0),( \pm \sqrt{a / d}: 1))$ to $(1: 0: 0)$. The completed curve also maps onto the inverted curve via $((X: Z),(Y: T)) \mapsto(Y Z: X T: X Y)$, but this map sends the two points $((0: 1),( \pm 1: 1))$ to $(1: 0: 0)$, and sends the two points $(( \pm 1: 1),(0: 1))$ to $(0: 1: 0)$. 
EECM-MPFQ uses the completed curve as an intermediate output of doublings (costing $4 \mathbf{S}+1 \mathbf{A})$ and additions (costing $5 \mathbf{M}+1 \mathbf{A})$; it then maps the completed point to a projective point (costing $3 \mathbf{M}$ ) or to an extended point (costing $4 \mathbf{M}$ ) as desired. One should not think that all addition formulas in the literature naturally factor through the completed curve: in particular, a detour through the completed curve would sacrifice $1 \mathbf{M}$ in the inverted Edwards addition law and in the projective dual addition law.

2.8. Addition with small inputs. There are two compatible ways to choose "small" curves that save more time in scalar multiplication. First, choosing small curve parameters $a, d$ speeds up any multiplications by those parameters inside addition formulas and doubling formulas. Second, choosing a small base point $P_{1}$ for scalar multiplication speeds up multiplications by the coordinates of $P_{1}$, and to some extent speeds up multiplications by the coordinates of [3] $P_{1}$ etc.

Let $P_{1}=\left(x_{1}, y_{1}\right)$ be a rational point on the Edwards curve $\mathrm{E}_{\mathrm{E}, 1, \bar{d}}$, and assume that $x_{1}, y_{1}, \bar{d}$ have small height, i.e., small numerators and denominators. Then $\bar{d}$ can be written in the form $d / a$, where $a$ is a small square and $d$ is a small integer. Now the point $\left(x_{1} / \sqrt{a}, y_{1}\right)$ is on the isomorphic curve $\mathrm{E}_{\mathrm{E}, a, d}$ and can be written with small integer coordinates on the inverted curve, the extended curve, etc., saving time in addition. A small inverted point $\left(X_{1}: Y_{1}: Z_{1}\right)$ replaces $4 \mathbf{M}$ by 4 small multiplications, specifically 1 multiplication by each of the small integers $X_{1}, Y_{1}, X_{1}+Y_{1}$, and $Z_{1}$; similarly, a small extended point replaces $5 \mathrm{M}$ by 5 small multiplications.

2.9. The Edwards group. If $a=1$ and $d$ is not a square then, by [13, Theorem $3.3]$, the affine Edwards addition law is complete: the denominators $1+d x_{1} x_{2} y_{1} y_{2}$ and $1-d x_{1} x_{2} y_{1} y_{2}$ are always nonzero, and the affine points $\left(x_{1}, y_{1}\right)$ on the curve form a group.

However, if $d$ is a square, then the addition law is not necessarily a group law: there can be pairs $\left(x_{1}, y_{1}\right)$ and $\left(x_{2}, y_{2}\right)$ where $1+d x_{1} x_{2} y_{1} y_{2}=0$ or $1-d x_{1} x_{2} y_{1} y_{2}=0$. Even worse, there can be pairs $\left(x_{1}, y_{1}\right)$ and $\left(x_{2}, y_{2}\right)$ for which $1+d x_{1} x_{2} y_{1} y_{2}=0=$ $x_{1} y_{2}+y_{1} x_{2}$ or $1-d x_{1} x_{2} y_{1} y_{2}=0=y_{1} y_{2}-a x_{1} x_{2}$. Switching from affine coordinates to projective or inverted or extended or completed coordinates does not allow the Edwards addition law to add such points.

Fortunately, on the completed curve $\overline{\mathrm{E}}_{\mathrm{E}, a, d}$ in $\mathbf{P}^{1} \times \mathbf{P}^{1}$, any pair of input points that cannot be added by the Edwards addition law can be added by the dual addition law. Gluing together the two addition laws produces a group law + : $\overline{\mathrm{E}}_{\mathrm{E}, a, d} \times \overline{\mathrm{E}}_{\mathrm{E}, a, d} \rightarrow \overline{\mathrm{E}}_{\mathrm{E}, a, d}$. This is the main result of [16]: the Edwards addition law and the dual addition law form a complete system of addition laws for $\overline{\mathrm{E}}_{\mathrm{E}, a, d}$.

The following theorem summarizes the results from 16. Section 3 uses this group law to characterize points of small order in $\overline{\mathrm{E}}_{\mathrm{E}, a, d}$, and subsequent sections of this paper use this characterization to construct Edwards curves with large Qtorsion groups.

Theorem 2.10. Fix a field $k$ with $\operatorname{char}(k) \neq 2$. Fix distinct nonzero elements $a, d \in k$. Fix $P_{1}, P_{2} \in \overline{\mathrm{E}}_{\mathrm{E}, a, d}(k)$. Write $P_{1}$ as $\left(\left(X_{1}: Z_{1}\right),\left(Y_{1}: T_{1}\right)\right)$ and write $P_{2}$ as 
$\left(\left(X_{2}: Z_{2}\right),\left(Y_{2}: T_{2}\right)\right)$. Define

$$
\begin{aligned}
X_{3} & =X_{1} Y_{2} Z_{2} T_{1}+X_{2} Y_{1} Z_{1} T_{2}, \\
Z_{3} & =Z_{1} Z_{2} T_{1} T_{2}+d X_{1} X_{2} Y_{1} Y_{2}, \\
Y_{3} & =Y_{1} Y_{2} Z_{1} Z_{2}-a X_{1} X_{2} T_{1} T_{2}, \\
T_{3} & =Z_{1} Z_{2} T_{1} T_{2}-d X_{1} X_{2} Y_{1} Y_{2} ;
\end{aligned}
$$

and

$$
\begin{aligned}
X_{3}^{\prime} & =X_{1} Y_{1} Z_{2} T_{2}+X_{2} Y_{2} Z_{1} T_{1}, \\
Z_{3}^{\prime} & =a X_{1} X_{2} T_{1} T_{2}+Y_{1} Y_{2} Z_{1} Z_{2}, \\
Y_{3}^{\prime} & =X_{1} Y_{1} Z_{2} T_{2}-X_{2} Y_{2} Z_{1} T_{1}, \\
T_{3}^{\prime} & =X_{1} Y_{2} Z_{2} T_{1}-X_{2} Y_{1} Z_{1} T_{2} .
\end{aligned}
$$

Then $X_{3} Z_{3}^{\prime}=X_{3}^{\prime} Z_{3}$ and $Y_{3} T_{3}^{\prime}=Y_{3}^{\prime} T_{3}$. Furthermore, at least one of the following cases occurs:

- $\left(X_{3}, Z_{3}\right) \neq(0,0)$ and $\left(Y_{3}, T_{3}\right) \neq(0,0)$. Then $P_{1}+P_{2}=\left(\left(X_{3}: Z_{3}\right)\right.$, $\left.\left(Y_{3}: T_{3}\right)\right)$.

- $\left(X_{3}^{\prime}, Z_{3}^{\prime}\right) \neq(0,0)$ and $\left(Y_{3}^{\prime}, T_{3}^{\prime}\right) \neq(0,0)$. Then $P_{1}+P_{2}=\left(\left(X_{3}^{\prime}: Z_{3}^{\prime}\right)\right.$, $\left.\left(Y_{3}^{\prime}: T_{3}^{\prime}\right)\right)$.

If $P_{1}=P_{2}$, then the first case occurs.

A completely different way to define the same group law on $\overline{\mathrm{E}}_{\mathrm{E}, a, d}$ is through a correspondence to the traditional chord-and-tangent elliptic-curve group law on an isomorphic Weierstrass curve. An explicit group isomorphism appears in 16, Theorems 7.1 and 7.3]. This correspondence has the disadvantage of requiring more case distinctions and more computations, but the advantage of making clear that the Edwards group is an elliptic-curve group. We use this to apply Mazur's theorem starting in Section 6 .

\section{Points of Small order on $\overline{\mathrm{E}}_{\mathrm{E}}, a, d$}

The complete set of addition laws from [16] (presented in the previous section) enables us to investigate the order of any point. In particular, it has often been stated that the points at infinity on an Edwards curve blow up to two points of order 2 and two points of order 4, e.g. in [8] in the context of exceptional points of the map between a twisted Edwards curve and a Montgomery curve. With the complete set of addition laws we can prove all statements purely in the context of Edwards curves.

This section characterizes all points of order 2, 3, and 4, and states conditions on the parameters of the twisted Edwards curve for such points to exist. These results are used later to construct curves with large Q-torsion groups. This section also characterizes points of order 8 relevant to later sections.

The following theorem gives a complete study of points of order 2 and 4 in $\overline{\mathrm{E}}_{\mathrm{E}, a, d}$.

Theorem 3.1. Fix a field $k$ with $\operatorname{char}(k) \neq 2$. Fix distinct nonzero elements $a, d \in k$. The following points are in $\overline{\mathrm{E}}_{\mathrm{E}, a, d}(k)$ and have the stated orders.

\section{Points of order 2:}

The point $((0: 1),(-1: 1))$ has order 2 .

If $a / d$ is a square in $k$, then the points $((1: 0),( \pm \sqrt{a / d}: 1))$ have order 2 .

There are no other points of order 2. 
Points of order 4 doubling to $((0: 1),(-1: 1))$ :

If $a$ is a square in $k$, then the points $((1: \pm \sqrt{a}),(0: 1))$ have order 4 and double to $((0: 1),(-1: 1))$.

If $d$ is a square in $k$, then the points $((1: \pm \sqrt{d}),(1: 0))$ have order 4 and double to $((0: 1),(-1: 1))$.

There are no other points doubling to $((0: 1),(-1: 1))$.

Points of order 4 doubling to $((1: 0),( \pm \sqrt{a / d}: 1))$ : Assume that $s \in k$ satisfies $s^{2}=a / d$.

If $s$ and $-s / a$ are squares in $k$, then the points $(( \pm \sqrt{-s / a}: 1),( \pm \sqrt{s}: 1))$, where the signs may be chosen independently, have order 4 and double to $((1: 0),(s: 1))$.

There are no other points doubling to $((1: 0),(s: 1))$.

Proof. Doublings can always be computed by $X_{3}, Z_{3}, Y_{3}, T_{3}$ from Theorem 2.10, in other words, all curve points $((X: Z),(Y: T))$ have $\left(2 X Y Z T, Z^{2} T^{2}+d X^{2} Y^{2}\right) \neq$ $(0,0)$ and $\left(Y^{2} Z^{2}-a X^{2} T^{2}, Z^{2} T^{2}-d X^{2} Y^{2}\right) \neq(0,0)$, so

$$
\begin{aligned}
& {[2]((X: Z),(Y: T))} \\
& \quad=\left(\left(2 X Y Z T: Z^{2} T^{2}+d X^{2} Y^{2}\right),\left(Y^{2} Z^{2}-a X^{2} T^{2}: Z^{2} T^{2}-d X^{2} Y^{2}\right)\right) .
\end{aligned}
$$

In particular:

- $[2]((0: 1),(-1: 1))=((0: 1),(1: 1))$.

- $[2]((1: 0),( \pm \sqrt{a / d}: 1))=((0: \ldots),(-a:-d(a / d)))=((0: 1),(1: 1))$.

- $[2]((1: \pm \sqrt{a}),(0: 1))=((0: \ldots),(-a: a))=((0: 1),(-1: 1))$.

- $[2]((1: \pm \sqrt{d}),(1: 0))=((0: \ldots),(d:-d))=((0: 1),(-1: 1))$.

- $[2](( \pm \sqrt{-s / a}: 1),( \pm \sqrt{s}: 1))=((\ldots: 1+d(-s / a) s),(s-a(-s / a): 1-$ $d(-s / a) s))=((1: 0),(s: 1))$ since $d(s / a) s=s^{2} d / a=1$.

To see that there is no other point of order 2 or 4 , observe first that every point $((X: Z),(Y: T))$ on $\overline{\mathrm{E}}_{\mathrm{E}, a, d}$ with $X=0$ or $Y=0$ or $Z=0$ or $T=0$ is either $((0: 1),(1: 1))$ or one of the points doubled above. The only remaining points are affine points $((x: 1),(y: 1))$ with $x \neq 0$ and $y \neq 0$. The double of $((x: 1),(y: 1))$ is $\left(\left(2 x y: 1+d x^{2} y^{2}\right),\left(y^{2}-a x^{2}: 1-d x^{2} y^{2}\right)\right)$; but $2 x y \neq 0$, so this double cannot be $((0: 1),(1: 1))$, so $((x: 1),(y: 1))$ cannot have order 2 . For the same reason, the double cannot be $((0: 1),(-1: 1))$. The only remaining case is that the double is $((1: 0),(s: 1))$ where $s^{2}=a / d$. Then $a x^{2}+y^{2}=1+d x^{2} y^{2}=0$ so $a x^{2}=-y^{2}$; and $y^{2}-a x^{2}=s\left(1-d x^{2} y^{2}\right)$, so $2 y^{2}=y^{2}-a x^{2}=s\left(1-d x^{2} y^{2}\right)=2 s$, so $y= \pm \sqrt{s}$, and finally $a x^{2}=-s$ so $x= \pm \sqrt{-s / a}$.

Later we will study Edwards curves over the rationals $\mathbf{Q}$ for which $((1: \pm \sqrt{a}),(0$ : $1))$ is on the curve. In this case the only points of order 8 double to either these points or to $((1: \pm \sqrt{d}),(1: 0))$.

Theorem 3.2. Fix a field $k$ with $\operatorname{char}(k) \neq 2$. Fix distinct nonzero elements $a, d \in k$.

Points of order 8 doubling to $((1: \pm \sqrt{a}),(0: 1)):$ If $r \in k$ satisfies $r^{2}=$ $a$, then any element of $\overline{\mathrm{E}}_{\mathrm{E}, a, d}(k)$ doubling to $((1: r),(0: 1))$ can be written as $\left(\left(x_{8}: 1\right),\left(r x_{8}: 1\right)\right)$ for some $x_{8} \in k$ satisfying ad $x_{8}^{4}-2 a x_{8}^{2}+1=0$.

Conversely, if $r, x_{8} \in k$ satisfy $r^{2}=a$ and $a d x_{8}^{4}-2 a x_{8}^{2}+1=0$, then the two points $\left(\left( \pm x_{8}: 1\right),\left( \pm r x_{8}: 1\right)\right)$, with matching signs, have order 8 and double to $((1: r),(0$ : $1))$. If also $d$ is a square in $k$, then the two points $\left(\left(1: \pm r x_{8} \sqrt{d}\right),\left(1: \pm x_{8} \sqrt{d}\right)\right)$, 
with matching signs, have order 8 , double to $((1: r),(0: 1))$, and are different from $\left(\left( \pm x_{8}: 1\right),\left( \pm r x_{8}: 1\right)\right)$. There are no other points doubling to $((1: r),(0: 1))$.

Points of order 8 doubling to $((1: \pm \sqrt{d}),(1: 0)):$ If $s \in k$ satisfies $s^{2}=$ $d$, then any element of $\overline{\mathrm{E}}_{\mathrm{E}, a, d}(k)$ doubling to $((1: s),(1: 0))$ can be written as $\left(\left(\bar{x}_{8}: 1\right),\left(1: s \bar{x}_{8}\right)\right)$ for some $\bar{x}_{8} \in k$ satisfying $a d \bar{x}_{8}^{4}-2 d \bar{x}_{8}^{2}+1=0$.

Conversely, if $s, \bar{x}_{8} \in k$ satisfy $s^{2}=d$ and ad $\bar{x}_{8}^{4}-2 d \bar{x}_{8}^{2}+1=0$, then the two points $\left(\left( \pm \bar{x}_{8}: 1\right),\left(1: \pm s \bar{x}_{8}\right)\right)$, with matching signs, have order 8 and double to $((1: s),(1:$ $0))$. If also $a$ is a square in $k$, then the two points $\left(\left(1: \pm s x_{8} \sqrt{a}\right),\left( \pm x_{8} \sqrt{a}: 1\right)\right)$, with matching signs, have order 8 , double to $((1: s),(1: 0))$, and are different from $\left(\left( \pm \bar{x}_{8}: 1\right),\left(1: \pm s \bar{x}_{8}\right)\right)$. There are no other points doubling to $((1: s),(1: 0))$.

Proof. Every point with a zero coordinate has order at most 4 by Theorem 3.1 so any point of order 8 has the form $\left(\left(x_{8}: 1\right),\left(y_{8}: 1\right)\right)$, with $x_{8} \neq 0$ and $y_{8} \neq 0$, and with double $\left(\left(2 x_{8} y_{8}: 1+d x_{8}^{2} y_{8}^{2}\right),\left(y_{8}^{2}-a x_{8}^{2}: 1-d x_{8}^{2} y_{8}^{2}\right)\right)$.

Part 1: If the double is $((1: r),(0: 1))$, then $y_{8}^{2}-a x_{8}^{2}=0$ and $2 x_{8} y_{8} r=$ $1+d x_{8}^{2} y_{8}^{2}=a x_{8}^{2}+y_{8}^{2}=2 a x_{8}^{2}=2 r^{2} x_{8}^{2}$. Cancel $2 x_{8} r$ to see that $y_{8}=r x_{8}$. Hence $a d x_{8}^{4}-2 a x_{8}^{2}+1=d x_{8}^{2} y_{8}^{2}-\left(1+d x_{8}^{2} y_{8}^{2}\right)+1=0$ and the original point is $\left(\left(x_{8}: 1\right),\left(r x_{8}: 1\right)\right)$.

Conversely, if $r, x_{8} \in k$ satisfy $r^{2}=a$ and $a d x_{8}^{4}-2 a x_{8}^{2}+1=0$, then the point $\left(\left(x_{8}: 1\right),\left(r x_{8}: 1\right)\right)$ is on the curve since $a x_{8}^{2}+\left(r x_{8}\right)^{2}=2 a x_{8}^{2}=a d x_{8}^{4}+1=$ $1+d x_{8}^{2}\left(r x_{8}\right)^{2}$, and it doubles to $\left(\left(2 x_{8} r x_{8}: 1+d x_{8}^{2} r^{2} x_{8}^{2}\right),\left(r^{2} x_{8}^{2}-a x_{8}^{2}: \ldots\right)\right)=$ $\left(\left(2 x_{8} r x_{8}: 2 a x_{8}^{2}\right),(0: \ldots)\right)=((1: r),(0: 1))$.

The other points doubling to $((1: r),(0: 1))$ are $((x: 1),(r x: 1))$ for other $x \in k$ satisfying $a d x^{4}-2 a x^{2}+1=0$. If $d$ is not a square in $k$, then $a d x^{4}-2 a x^{2}+1=a d x^{4}-$ $\left(a d x_{8}^{2}+1 / x_{8}^{2}\right) x^{2}+1=\left(x-x_{8}\right)\left(x+x_{8}\right)\left(a d x^{2}-1 / x_{8}^{2}\right)$, with $a d x^{2}-1 / x_{8}^{2}$ irreducible, so the only points doubling to $((1: r),(0: 1))$ are $\left(\left( \pm x_{8}: 1\right),\left( \pm r x_{8}: 1\right)\right)$. If $d$ is a square in $k$, then $a d x^{4}-2 a x^{2}+1=\left(x-x_{8}\right)\left(x+x_{8}\right)\left(r x \sqrt{d}-1 / x_{8}\right)\left(r x \sqrt{d}+1 / x_{8}\right)$ so the only points doubling to $((1: r),(0: 1))$ are $\left(\left( \pm x_{8}: 1\right),\left( \pm r x_{8}: 1\right)\right)$ and $\left(\left(1: \pm r x_{8} \sqrt{d}\right),\left(1: \pm x_{8} \sqrt{d}\right)\right)$. These points are distinct: otherwise $\pm r x_{8}^{2} \sqrt{d}=1$, so $a d x_{8}^{4}=1$, so $2 a x_{8}^{2}=2$, so $a x_{8}^{2}=1$, so $y_{8}=0$ from the curve equation, contradiction.

Part 2: If the double of $\left(\left(\bar{x}_{8}: 1\right),\left(\bar{y}_{8}: 1\right)\right)$ is $((1: s),(1: 0))$, then $1-d \bar{x}_{8}^{2} \bar{y}_{8}^{2}=0$ and $2 \bar{x}_{8} \bar{y}_{8} s=1+d \bar{x}_{8}^{2} \bar{y}_{8}^{2}=2$ so $\bar{y}_{8}=1 /\left(s \bar{x}_{8}\right)$. Hence $a d \bar{x}_{8}^{4}-2 d \bar{x}_{8}^{2}+1=\left(a \bar{x}_{8}^{2}-2+\right.$ $\left.\bar{y}_{8}^{2}\right) d \bar{x}_{8}^{2}=0$ and the original point is $\left(\left(\bar{x}_{8}: 1\right),\left(1: s \bar{x}_{8}\right)\right)$.

Conversely, if $s, \bar{x}_{8} \in k$ satisfy $s^{2}=d$ and $a d \bar{x}_{8}^{4}-2 d \bar{x}_{8}^{2}+1=0$, then the point $\left(\left(\bar{x}_{8}: 1\right),\left(1: s \bar{x}_{8}\right)\right)$ is on the curve since $d \bar{x}_{8}^{2}\left(a \bar{x}_{8}^{2}+\bar{y}_{8}^{2}\right)=d \bar{x}_{8}^{2}\left(a \bar{x}_{8}^{2}+1 /\left(s^{2} \bar{x}_{8}^{2}\right)\right)=$ $a d \bar{x}_{8}^{4}+1=2 d \bar{x}_{8}^{2}=d \bar{x}_{8}^{2}+d \bar{x}_{8}^{4} / \bar{x}_{8}^{2}=d \bar{x}_{8}^{2}\left(1+d \bar{x}_{8}^{2} /\left(s^{2} \bar{x}_{8}^{2}\right)\right)=d \bar{x}_{8}^{2}\left(1+d \bar{x}_{8}^{2} \bar{y}_{8}^{2}\right)$. The point doubles to $\left(\left(2 s \bar{x}_{8}^{2}: s^{2} \bar{x}_{8}^{2}+d \bar{x}_{8}^{2}\right),\left(1-a s^{2} \bar{x}_{8}^{4}: s^{2} \bar{x}_{8}^{2}-d \bar{x}_{8}^{2}\right)\right)=\left((1: s),\left(1-a d \bar{x}_{8}^{4}\right.\right.$ : $\left.\left.s^{2} \bar{x}_{8}^{2}-s^{2} \bar{x}_{8}^{2}\right)\right)=((1: s),(1: 0))$.

The other points doubling to $((1: s),(1: 0))$ are $((x: 1),(1: s x))$ for other $x \in k$ satisfying $a d x^{4}-2 d x^{2}+1=0$. If $a$ is not a square in $k$, then $a d x^{4}-2 d x^{2}+1=a d x^{4}-$ $\left(a d \bar{x}_{8}^{2}+1 / \bar{x}_{8}^{2}\right) x^{2}+1=\left(x-\bar{x}_{8}\right)\left(x+\bar{x}_{8}\right)\left(a d x^{2}-1 / \bar{x}_{8}^{2}\right)$, with $a d x^{2}-1 / \bar{x}_{8}^{2}$ irreducible, so the only points doubling to $((1: s),(1: 0))$ are $\left(\left( \pm \bar{x}_{8}: 1\right),\left(1: \pm s \bar{x}_{8}\right)\right)$. If $a$ is a square in $k$, then $a d x^{4}-2 d x^{2}+1=\left(x-\bar{x}_{8}\right)\left(x+\bar{x}_{8}\right)\left(s x \sqrt{a}-1 / \bar{x}_{8}\right)\left(s x \sqrt{a}+1 / \bar{x}_{8}\right)$ so the only points doubling to $((1: s),(1: 0))$ are $\left(\left( \pm \bar{x}_{8}: 1\right),\left(1: \pm s \bar{x}_{8}\right)\right)$ and $\left.\left(\left(1: \pm s \bar{x}_{8} \sqrt{a}\right)\right),\left( \pm \bar{x}_{8} \sqrt{a}: 1\right)\right)$. These points are distinct: otherwise $\pm s \bar{x}_{8}^{2} \sqrt{a}=$ 1 so $a d \bar{x}_{8}^{4}=1$ so $2 d \bar{x}_{8}^{2}=2$ so $d \bar{x}_{8}^{2}=1$ so $\bar{x}_{8}=0$ from the curve equation, contradiction. 
Theorem 3.3. Fix a field $k$ with $\operatorname{char}(k) \neq 2$. Fix distinct nonzero elements $a, d \in k$. If $x_{3}, y_{3} \in k$ satisfy $a x_{3}^{2}+y_{3}^{2}=1+d x_{3}^{2} y_{3}^{2}=-2 y_{3}$, then $\left(\left(x_{3}: 1\right),\left(y_{3}: 1\right)\right)$ is a point of order 3 on $\overline{\mathrm{E}}_{\mathrm{E}, a, d}(k)$. Conversely, all points of order 3 on $\overline{\mathrm{E}}_{\mathrm{E}, a, d}(k)$ arise in this way.

Proof. Doublings can always be computed by $X_{3}, Z_{3}, Y_{3}, T_{3}$ from Theorem 2.10 , as in the proof of Theorem 3.1

Observe that $\left(\left(x_{3}: 1\right),\left(y_{3}: 1\right)\right) \in \overline{\mathrm{E}}_{\mathrm{E}, a, d}(k)$ since $a x_{3}^{2}+y_{3}^{2}=1+d x_{3}^{2} y_{3}^{2}$. Now

$$
\begin{aligned}
{[2]\left(\left(x_{3}: 1\right),\left(y_{3}: 1\right)\right) } & =\left(\left(2 x_{3} y_{3}: 1+d x_{3}^{2} y_{3}^{2}\right),\left(y_{3}^{2}-a x_{3}^{2}: 1-d x_{3}^{2} y_{3}^{2}\right)\right) \\
& =\left(\left(2 x_{3} y_{3}:-2 y_{3}\right),\left(2 y_{3}^{2}+2 y_{3}: 2 y_{3}+2\right)\right) \\
& =\left(\left(-x_{3}: 1\right),\left(y_{3}: 1\right)\right)
\end{aligned}
$$

so $\left(\left(x_{3}: 1\right),\left(y_{3}: 1\right)\right)$ has order dividing 3 . It cannot have order 1 (since otherwise $x_{3}=0$ so $\left.y_{3}^{2}=1=-2 y_{3}\right)$, so it has order 3 .

Conversely, consider any point $P=\left(\left(X_{1}: Z_{1}\right),\left(Y_{1}: T_{1}\right)\right)$ of order 3 in $\overline{\mathrm{E}}_{\mathrm{E}, a, d}(k)$. The equation [2] $P=-P$ then implies $\left(2 X_{1} Y_{1} Z_{1} T_{1}: Z_{1}^{2} T_{1}^{2}+d X_{1}^{2} Y_{1}^{2}\right)=\left(-X_{1}: Z_{1}\right)$. Every point in $\overline{\mathrm{E}}_{\mathrm{E}, a, d}$ with a zero coordinate has order 1,2 , or 4 by Theorem 3.1 . so $X_{1}, Z_{1}, Y_{1}, T_{1} \neq 0$. Define $x_{3}=X_{1} / Z_{1}$ and $y_{3}=Y_{1} / T_{1}$. Then $P=\left(\left(x_{3}: 1\right),\left(y_{3}\right.\right.$ : $1)$ ); furthermore $\left(2 x_{3} y_{3}: 1+d x_{3}^{2} y_{3}^{2}\right)=\left(-x_{3}: 1\right)$ and $x_{3} \neq 0$ so $-2 y_{3}=1+d x_{3}^{2} y_{3}^{2}=$ $a x_{3}^{2}+y_{3}^{2}$.

\section{Using EDWARdS CURVES IN ECM STAGE 1}

This section discusses "stage 1" of ECM. It begins by reviewing the general idea of stage 1 and the state-of-the-art strategies used in GMP-ECM to perform the elliptic-curve computations in stage 1. It then analyzes the speedups obtained from using Edwards curves.

4.1. Overview of stage 1. Stage 1 of ECM tries to factor a positive integer $n$ as follows. Choose an elliptic curve $E$ defined over $\mathbf{Q}$, and a nontorsion element $P \in E(\mathbf{Q})$. Choose a rational function $\phi: E \rightarrow \mathbf{Q}$ that has a pole at the neutral element of $E$; for example, choose $\phi$ as the Weierstrass $x$-coordinate. Choose a positive integer $s$ with many small prime factors. Choose a sequence of additions, subtractions, multiplications, and divisions that, if carried out over $\mathbf{Q}$, would compute $\phi([s] P)$, where $[s] P$ denotes the $s$ th multiple of $P$ in $E(\mathbf{Q})$. Compute $\phi([s] P)$ modulo $n$ by carrying out this sequence of additions, subtractions, multiplications, and divisions modulo $n$. Hope for an impossible division modulo $n$. An attempt to divide by a nonzero nonunit modulo $n$ immediately reveals a factor of $n$; an attempt to divide by 0 modulo $n$ is not quite as informative but usually allows a factor of $n$ to be obtained without much extra work.

If $n$ has a prime divisor $q$ such that $[s] P$ is the neutral element of $E(\mathbf{Z} / q \mathbf{Z})$, then the stage-1 ECM computation will involve an impossible division modulo $n$, usually revealing a factor of $n$. This occurs, in particular, whenever $s$ is a multiple of the group size $\# E(\mathbf{Z} / q \mathbf{Z})$. As $E$ varies randomly, $\# E(\mathbf{Z} / q \mathbf{Z})$ varies randomly (with some subtleties in its distribution; see, e.g., 35]) in the Hasse interval $[q-2 \sqrt{q}+$ $1, q+2 \sqrt{q}+1]$. What makes ECM useful is that a surprisingly small $s$, allowing a surprisingly fast computation of $[s] P$, is a multiple of a surprisingly large fraction of the integers in the Hasse interval, and is a multiple of the order of $P$ modulo $q$ with (conjecturally) an even larger probability. See Section 9 for detailed statistics. 
For example, one could try to factor $n$ as follows. Choose the curve $E: y^{2}=$ $x^{3}-2$, the Weierstrass $x$-coordinate as $\phi$, the point $(x, y)=(3,5)$, and the integer $s=420$. Choose the following strategy to compute the $x$-coordinate of $[420](3,5)$ : use the standard affine-coordinate doubling formulas to compute $[2](3,5)$, then $[4](3,5)$, then $[8](3,5)$; use the standard affine-coordinate addition formulas to compute $[12](3,5)$; continue similarly through $[2](3,5),[4](3,5),[8](3,5),[12](3,5)$, $[24](3,5),[48](3,5),[96](3,5),[192](3,5),[384](3,5),[408](3,5),[420](3,5)$. Carry out these computations modulo $n$, hoping for a division by a nonzero nonunit modulo $n$.

The denominator of the $x$-coordinate of $[420](3,5)$ in $E(\mathbf{Q})$ has many small prime factors: $2,3,5,7,11,19,29,31,41,43,59,67,71,83,89,109,163,179,181,211$, $223,241,269,283,383,409,419,433,523,739,769,811,839$, etc. If $n$ shares any of these prime factors, then the computation of $[420](3,5)$ will encounter an impossible division modulo $n$. To verify the presence of (e.g.) the primes 769,811 , and 839, one can observe that $[420](3,5)$ is the neutral element in each of the groups $E(\mathbf{Z} / 769 \mathbf{Z}), E(\mathbf{Z} / 811 \mathbf{Z}), E(\mathbf{Z} / 839 \mathbf{Z})$; the order of $(3,5)$ turns out to be $7,42,35$, respectively. Note that the group orders are 819,756 , and 840 , none of which divide 420.

4.2. The standard choice of $s$. Pollard in [40, page 527] suggested choosing $s$ as "the product of all the primes $p_{i} \leq L$ each to some power $c_{i} \geq 1$. There is some freedom in the choice of the $c_{i}$ but the smallest primes should certainly occur to some power higher than the first."

Pollard's prime bound " $L$ " is now called $B_{1}$. One possibility is to choose, for each prime $\pi \leq B_{1}$, the largest power of $\pi$ in the interval $[1, n+2 \sqrt{n}+1]$. Then $[s] P$ is the neutral element in $E(\mathbf{Z} / q \mathbf{Z})$ if and only if the order of $P$ is " $B_{1}$-smooth", i.e., if and only if the order has no prime divisors larger than $B_{1}$. This possibility is theoretically pleasing but clearly suboptimal.

Brent in [18, Section 5] said that "in practice we choose" the largest power of $\pi$ in the interval $\left[1, B_{1}\right]$ "because this significantly reduces the cost of a trial without significantly reducing the probability of success." In other words, $s=$ $\operatorname{lcm}\left\{1,2, \ldots, B_{1}\right\}$. GMP-ECM uses the same strategy; see [47, page 529].

4.3. The standard prime-by-prime strategy. Pollard in [40, page 527] said that one "can choose between using the primes $p_{i}$ in succession or computing $P$ in advance and performing a single power operation." Pollard's " $P$ " is $s$ in the notation of this paper.

As far as we know, all ECM implementations use the first strategy, working with one prime at a time. Brent in [18, Section 5] wrote, "Actually, $E$ [i.e., $s$ in the notation of this paper] is not computed. Instead ... repeated operations of the form $P:=P^{k}$ [i.e., $[k] P$ in the notation of this paper], where $k \ldots$ is a prime power." Montgomery in [36, page 249] wrote, "It is unnecessary to compute $R$ [i.e., $s$ in the notation of this paper] explicitly." Zimmermann and Dodson in [47, page 529] wrote, "That big product is not computed as such" and presented the prime-by-prime loop used in GMP-ECM.

4.4. The standard elliptic-curve coordinate system. Chudnovsky and Chudnovsky in [20, Section 4] wrote, "The crucial problem becomes the choice of the model of an algebraic group variety, where computations mod $p$ are the least time 
consuming." They presented explicit formulas for computations on several different shapes of elliptic curves.

Montgomery in [36, Section 10.3.1] introduced what are now called "Montgomery coordinates": a point $\left(x_{1}, y_{1}\right)$ on the elliptic curve $B y^{2}=x^{3}+A x^{2}+x$ is represented as a pair $\left(X_{1}: Z_{1}\right)$ such that $X_{1} / Z_{1}=x_{1}$. This representation does not distinguish $\left(x_{1}, y_{1}\right)$ from $\left(x_{1},-y_{1}\right)$, so it does not allow addition, but it does allow "differential addition," i.e., computation of $P+Q$ given $P, Q, P-Q$. In particular, Montgomery presented explicit formulas to compute $P,[2 k] P,[(2 k+1)] P$ from $P,[k] P,[k+1] P$ using $6 \mathbf{M}+4 \mathbf{S}+1 \mathbf{C}$, or $5 \mathbf{M}+4 \mathbf{S}+1 \mathbf{C}$ if $P$ is given with $Z_{1}=1$, or $4 \mathbf{M}+4 \mathbf{S}+1 \mathbf{C}$ if $P$ is a very small point such as $\left(X_{1}: Z_{1}\right)=(2: 1)$. Here $\mathbf{C}$ stands for the cost of multiplication by $(A+2) / 4$. One can find earlier formulas for the same computation in [20. formula (4.19)], but Montgomery's formulas are faster.

As far as we know, all subsequent ECM implementations have used Montgomery coordinates. In particular, GMP-ECM uses Montgomery coordinates for stage 1, with "PRAC," a particular differential addition chain introduced by Montgomery. Zimmermann and Dodson in [47, page 532, Figure 2] report a total cost of 2193683 differential additions to multiply an elliptic-curve point by $2 \cdot 3 \cdot 5 \cdot 7 \cdot 11 \cdots 999983 \approx$ $2^{1440508.1677}$ in Montgomery coordinates. By adding a few counters to the source code of GMP-ECM we observed that GMP-ECM's stage 1, with $B_{1}=10^{6}$ and hence $s \approx 2^{1442098.6271}$, used 12982280 multiplications modulo $n$ for 2196070 elliptic-curve differential additions, of which only 194155 were doublings.

4.5. Speedups in EECM-MPFQ. EECM-MPFQ breaks with stage-1 tradition in three ways:

- EECM-MPFQ uses Edwards curves $x^{2}+y^{2}=1+d x^{2} y^{2}$ with extended Edwards coordinates with $\phi=1 / x$ whereas GMP-ECM uses Montgomery curves with Montgomery coordinates. See below for performance results. Our prototype GMP-EECM used inverted twisted Edwards coordinates.

- EECM-MPFQ and GMP-EECM handle the prime factors $\pi$ of $s$ in batches, whereas GMP-ECM handles each prime factor separately. Specifically, GMP-EECM computed the product $t$ of a batch, replaced $P$ with $[t] P$, and then moved on to the next batch. EECM-MPFQ always uses a single batch: it computes the entire product $s$ and then replaces $P$ with $[s] P$. The large batches save time, as discussed below; the computation of $s$ takes negligible time.

- EECM-MPFQ uses "signed sliding fractional window" addition-subtraction chains. Our prototype GMP-EECM used "signed sliding window" additionsubtraction chains. These chains compute $P \mapsto[s] P$ using only 1 doubling and $\epsilon$ additions for each bit of $s$. Here $\epsilon$ converges to 0 as $s$ increases in length; this is why larger batches save time. The savings are amplified by the fact that an addition is somewhat more expensive than a doubling. Note that these chains are not compatible with Montgomery coordinates; they are shorter than any differential addition chain can be.

EECM-MPFQ follows tradition in its choice of $s$. Our experiments have not found significant speedups from other choices of $s$ : for example, allowing prime powers in the larger interval $\left[1, B_{1}^{1.5}\right]$ has negligible extra cost when $B_{1}$ is large, but it also appears to have negligible benefit. 
The addition-subtraction chains used in EECM-MPFQ are the chains $C_{m}(s)$ defined in [15, Section 3]. Given $B_{1}$, EECM-MPFQ computes $s$, computes $C_{m}(s)$ for various choices of the chain parameter $m$, and keeps the lowest-cost chain that it finds in a simple measure of cost. (Variations in the cost measure do not lead to noticeably better chains.) The total time spent on this computation is small: for example, under a second (on the CPU described below) for $B_{1}=1048576$. The resulting chain is reused for many curves and many inputs $n$.

Table 4.1 shows the actual number of elliptic-curve doublings and additions used by stage 1 of EECM-MPFQ. Table 4.1 also shows the actual number of field squarings, field multiplications, and field additions used by stage 1 of EECM-MPFQ. Recall that each doubling uses $3 \mathbf{M}+4 \mathbf{S}$ while each addition uses $9 \mathbf{M}$. The table shows that EECM-MPFQ uses only 8.84 multiplications per bit of $s$ for $B_{1}=64$, only 8.42 multiplications per bit for $B_{1}=512$, only 7.91 multiplications per bit for $B_{1}=16384$, and only 7.61 multiplications per bit for $B_{1}=1048576$.

For comparison, GMP-ECM uses approximately 9 multiplications for each bit of $s$, as illustrated by the example with $B_{1}=10^{6}$ above. Zimmermann and Dodson explain in [47, Section 2] that one cannot expect differential addition chains to use fewer than $6 / \log _{2}((1+\sqrt{5}) / 2) \approx 8.64$ multiplications per bit. Furthermore, only about one third of GMP-ECM's multiplications are squarings, while more than half of EECM-MPFQ's multiplications are squarings for $B_{1} \geq 16384$. Even for the most carefully chosen curves, with extremely small parameters and extremely small base points, Montgomery's formulas use at least $4 \mathbf{M}+4 \mathbf{S}$ per bit.

4.6. Measurements of CPU cycles. GMP-ECM relies primarily on the GMP integer-arithmetic library developed by Granlund et al., although for some CPUs it replaces portions of GMP with its own assembly-language subroutines for modular multiplication. EECM-MPFQ also uses GMP but performs almost all modular arithmetic using the MPFQ library introduced by Gaudry and Thomé in [27]. The tests described below used GMP 4.3.1 (released May 2009), GMP-ECM 6.2.3 (released April 2009), and MPFQ 1.0rc1 (released September 2008), all current at the time of testing (November 2009).

A 1000-curve test of EECM-MPFQ took 2.8 million cycles per curve on a single core of a $3.2 \mathrm{GHz}$ AMD Phenom II X4 (100f42) for a 240-bit $n$ with $B_{1}=1024$ (and with $d_{1}=1$, disabling "stage 2"). For comparison, a 1000-curve test of GMP-ECM took 3.8 million cycles per curve on the same CPU for the same 240-bit $n$ with the same $B_{1}$ (and with $B_{2}=1$ ).

The improvement in speed from GMP-ECM to EECM-MPFQ is even larger than what one would expect from comparing GMP-ECM's 8512M + 4427S to EECMMPFQ's $6363 \mathrm{M}+5892 \mathrm{~S}$. The obvious explanation is that MPFQ's modular multiplications are faster than GMP's (and GMP-ECM's) modular multiplications; of course, the credit for this speedup belongs to Gaudry and Thomé.

Increasing $B_{1}$ to 16384 increased the EECM-MPFQ time to 40 million cycles per curve. There are 187307 modular multiplications per curve, specifically $92651 \mathrm{M}+$ $94656 \mathbf{S}$; evidently each modular multiplication took only about 220 cycles. For comparison, increasing $B_{1}$ to 16384 increased the GMP-ECM time to 60 million cycles per curve for 210307 modular multiplications, specifically $138884 \mathbf{M}+71423 \mathrm{~S}$.

Increasing $B_{1}$ to 65536 increased the EECM-MPFQ time to 162 million cycles per curve. There are 735618 modular multiplications per curve, specifically $357866 \mathrm{M}+$ $377752 \mathrm{~S}$. For comparison, increasing $B_{1}$ to 65536 increased the GMP-ECM time 
TABLE 4.1. Costs of computation of $s P$ in EECM-MPFQ. The $b$ column is the number of bits in $s=\operatorname{lcm}\left\{1,2, \ldots, B_{1}\right\}$. \#DBL and \#ADD are the number of

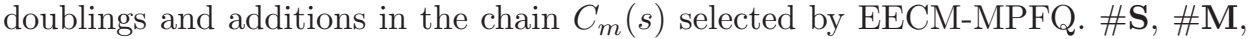
and $\# \mathbf{a}$ are the number of field squarings, field multiplications, and field additions used by these elliptic-curve operations in extended Edwards coordinates. Per-curve setup costs and precomputation costs are included in the field-operation counts.

\begin{tabular}{|c|c|c|c|c|c|c|c|c|}
\hline$B_{1}$ & $b$ & $m$ & $\frac{\# \mathrm{DBL}}{b}$ & $\frac{\# \mathrm{ADD}}{b}$ & $\frac{\# \mathbf{S}+\# \mathbf{M}}{b}$ & $\frac{\# \mathbf{S}}{b}$ & $\frac{\# \mathbf{M}}{b}$ & $\frac{\# \mathbf{a}}{b}$ \\
\hline 8 & 10 & 5 & 0.800000 & 0.400000 & 10.100000 & 3.200000 & 6.900000 & 7.600000 \\
\hline 12 & 15 & 3 & 933333 & 0.266667 & 9.533333 & 3.733333 & 5.800000 & 466667 \\
\hline 16 & 20 & 5 & 900000 & 0.250000 & 9.000000 & 3.600000 & 5.400000 & 7.150000 \\
\hline 24 & 33 & 3 & 939394 & 0.242424 & 9.030303 & 3.757576 & 5.272727 & 7.333333 \\
\hline 32 & 48 & 11 & 916667 & 0.291667 & 9.229167 & 3.666667 & 5.562500 & 7.541667 \\
\hline 48 & 69 & 7 & 956522 & 0.202899 & 8.652174 & 3.826087 & 4.826087 & 7.159420 \\
\hline 64 & 90 & 9 & 977778 & 0.211111 & 8.844444 & 3.911111 & 4.933333 & 7.344444 \\
\hline 96 & 130 & 15 & 969231 & 0.215385 & 8.792308 & 3.876923 & 4.915385 & 7.323077 \\
\hline 128 & 184 & 15 & 978261 & 0.201087 & 8.706522 & 3.913043 & 4.793478 & 7.277174 \\
\hline 192 & 275 & 29 & 985455 & 0.185455 & 8.600000 & 3. & 4.6 & 0909 \\
\hline 256 & 363 & 15 & 3981 & 0.190083 & & & 4.70 & 463 \\
\hline 384 & 55 & 27 & 1023 & 0.168761 & 8.472172 & 3.964093 & 4.508079 & 7.127469 \\
\hline 51 & & 27 & 993271 & 0.161 & & 3.973082 & 4.4 & 7.090175 \\
\hline 76 & 1106 & 63 & 994575 & 0.150995 & 8.329114 & 8300 & 4.350814 & 1412 \\
\hline 1024 & & 63 & 995943 & 0.145368 & 8.286004 & 3773 & 4.302231 & 93239 \\
\hline & & 115 & 996833 & 0.138462 & 8.228 & 3.987330 & 4.240724 & 0226 \\
\hline & 29 & 10 & 623 & 0.1 & 56 & 492 & 4.1 & 7980 \\
\hline & & 12 & 17 & 0.124041 & 8. & 3. & 4.1 & 0171 \\
\hline & $5 s$ & 23 & 0 & 0.120506 & 8.0 & 3.9 & 4. & 443 \\
\hline & & 2 & 8 & 0.1145 & 3 & 3. & $4 .(1$ & 752 \\
\hline & & 27 & 22 & & 35 & 87 & $4 .(1$ & 5621 \\
\hline 12288 & & 5 & 92 & 5287 & 06 & 3. & 3.9 & 958 \\
\hline & & 5 & 20 & 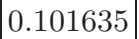 & 63 & 3.9 & 3.9 & 9162 \\
\hline 24576 & 3 & 877 & 19 & 2 & 65 & 3.9 & 3.8 & 0262 \\
\hline 32 & 30 & 1019 & 88 & 66 & 15 & 153 & 3.8 & 6.656490 \\
\hline & 70 & & 59 & 7 & 03 & 3. & & 0880 \\
\hline 65536 & 149 & & 884 & 98 & 21 & 3.999534 & 3.788987 & 6.613188 \\
\hline 98304 & 141805 & & 999922 & 84087 & .756278 & 3.999690 & 3.756588 & 6.588146 \\
\hline 131072 & 24 & 3079 & 999942 & 0.082057 & 7.738135 & 3.999767 & 3.738367 & 6.574052 \\
\hline 196608 & & 4115 & 999958 & 0.078692 & 7.707947 & 3.999831 & 3.708117 & 6.550589 \\
\hline 262144 & & & 999968 & 0.076815 & 20 & 3.999873 & 3.691255 & 6.537516 \\
\hline 39 & & & & & 99 & 3.9 & 3.664 & 6.517046 \\
\hline & & & & & & & 3.649 & 4745 \\
\hline & & & & & & & & 38054 \\
\hline 1048576 & 1512566 & 16389 & 0.999991 & 0.067937 & 7.611370 & 3.999963 & 3.611407 & 6.475503 \\
\hline
\end{tabular}

to 243 million cycles per curve for 842998 modular multiplications, specifically $557257 \mathrm{M}+285741 \mathrm{~S}$.

4.7. EECM vs. HECM. Chudnovsky and Chudnovsky in [20, Section 6] proposed a genus-2 hyperelliptic-curve method of factoring, using "simple forms of laws of addition on hyperelliptic surfaces, isogenous to the product of two elliptic curves." 
Recently, in 21], Cosset reported that streamlined genus-2 formulas by Gaudry in [26] used only 189667 multiplications per elliptic curve (performed as 379334 multiplications per genus- 2 curve) for $B_{1}=16384$, with the extra advantage that approximately $75 \%$ of the multiplications are squarings. Cosset quoted, for comparison, an earlier version of this paper that had reported 195111 multiplications per curve for GMP-EECM for $B_{1}=16384$.

A closer look shows that the formulas in [26] and 21] actually use, for each elliptic curve, 189667 multiplications plus approximately 189667 multiplications by small constants. EECM-MPFQ uses a total of only 187307 multiplications per elliptic curve, and the advantage grows as $B_{1}$ grows. Furthermore, the elliptic curves used in 21] are less effective than the elliptic curves used in EECM-MPFQ, and in fact are less effective than the elliptic curves used in GMP-ECM, according to the experiments described in [21, Section 3]. HECM is worth further investigation, but in its current form is clearly less efficient than EECM.

\section{Using EdWARds CURVES IN ECM STAGe 2}

This section discusses "stage 2" of ECM, and the benefit of switching to Edwards curves in stage 2 .

5.1. Overview of stage 2. Recall that stage 1 hopes for $n$ to have a prime divisor $q$ such that $[s] P$ is the neutral element of $E(\mathbf{Z} / q \mathbf{Z})$.

Stage 2 hopes for $n$ to have a prime divisor $q$ such that $[s] P$ has small prime order in $E(\mathbf{Z} / q \mathbf{Z})$ : specifically, order $\ell$ for some prime $\ell$ between $B_{1}$ and $B_{2}$. Here $B_{1}$ is the stage- 1 parameter described in the previous section, and $B_{2}$ is a new stage-2 parameter.

The most obvious way to check for a small order of $[s] P$ is a prime-by-prime approach, computing $[\ell s] P$ modulo $n$ for each prime $\ell$.

If $\ell^{\prime}$ is the next prime after $\ell$, then one can move from $[\ell s] P$ to $\left[\ell^{\prime} s\right] P$ by adding a precomputed point $\left[\left(\ell^{\prime}-\ell\right) s\right] P$. Computing all $[\ell s] P$ in this way takes about $B_{2} / \log B_{2}-B_{1} / \log B_{1}$ elliptic-curve additions modulo $n$ (where log without a subscript means the natural logarithm): there are about $B_{2} / \log B_{2}-B_{1} / \log B_{1}$ primes $\ell$, and the time for precomputation is quite small, since the differences $\ell^{\prime}-\ell$ are generally quite small.

5.2. Standard speedup: Baby steps and giant steps. A better way to check for a small order of $[s] P$ is with the following baby-step-giant-step computation. Fix a parameter $d_{1} \in\{2,4,6, \ldots\}$, preferably a product of several different tiny primes. Choose a rational function $\psi: E \rightarrow \mathbf{Q}$ satisfying $\psi([j s] P)=\psi([-j s] P)$; for example, choose $\psi$ as the Weierstrass $x$-coordinate or the Edwards $y$-coordinate. Compute

$$
\operatorname{gcd}\left\{n, \prod_{\left\lceil B_{1} / d_{1}-1 / 2\right\rceil \leq i \leq\left\lfloor B_{2} / d_{1}+1 / 2\right\rfloor} \prod_{\substack{1 \leq j \leq d_{1} / 2 \\ \operatorname{gcd}\left\{j, d_{1}\right\}=1}}\left(\psi\left(\left[i d_{1} s\right] P\right)-\psi([j s] P)\right)\right\} .
$$

The idea here is as follows. Assume that $\ell$ is a prime between $B_{1}$ and $B_{2}$ not dividing $d_{1}$. Write $\ell$ as $i d_{1} \pm j$ for some integers $i, j$ with $j \in\left\{0,1, \ldots, d_{1} / 2\right\}$. Then $i$ is between $B_{1} / d_{1}-1 / 2$ and $B_{2} / d_{1}+1 / 2$, and $\operatorname{gcd}\left\{j, d_{1}\right\}=\operatorname{gcd}\left\{\ell, d_{1}\right\}=1$. If $[s] P$ has order $\ell$ in $E(\mathbf{Z} / q \mathbf{Z})$, then $\left[i d_{1} s\right] P=[\mp j s] P$ in $E(\mathbf{Z} / q \mathbf{Z})$ so the numerator of $\psi\left(\left[i d_{1} s\right] P\right)-\psi([j s] P)$ is divisible by $q$. 
In particular, the number of $i$ 's is balanced with the number of $j$ 's when $B_{2}-B_{1} \approx$ $d_{1} \varphi\left(d_{1}\right) / 2$, where $\varphi$ is Euler's totient function. The baby steps $[j s] P$ and the giant steps $\left[i d_{1} s\right] P$ use about $\varphi\left(d_{1}\right)$ elliptic-curve additions, while the product of $\psi\left(\left[i d_{1} s\right] P\right)-\psi([j s] P)$ uses about $\varphi\left(d_{1}\right)^{2} / 4$ multiplications modulo $n$.

For comparison, the prime-by-prime approach uses roughly $d_{1} \varphi\left(d_{1}\right) /\left(4 \log d_{1}\right)$ elliptic-curve additions. The baby-step-giant-step approach is an improvement whenever an elliptic-curve addition costs more than about $\left(\varphi\left(d_{1}\right) \log d_{1}\right) / d_{1}$ multiplications.

Asymptotically, $\left(\varphi\left(d_{1}\right) \log d_{1}\right) / d_{1}$ reaches $\infty$, even when $d_{1}$ is chosen as a product of tiny primes. However, in practice, $\left(\varphi\left(d_{1}\right) \log d_{1}\right) / d_{1}$ is always below 4 ; for example, if $d_{1}=510510=2 \cdot 3 \cdot 5 \cdot 7 \cdot 11 \cdot 13 \cdot 17$, then $\left(\varphi\left(d_{1}\right) \log d_{1}\right) / d_{1} \approx 2.37$. The baby-step-giant-step approach is therefore faster than the prime-by-prime approach.

5.3. Standard speedup: Fast polynomial arithmetic. If $d_{1}$ is large, then $\prod_{i} \prod_{j}\left(\psi\left(\left[i d_{1} s\right] P\right)-\psi([j s] P)\right)$ is more efficiently computed as $\prod_{i} F\left(\psi\left(\left[i d_{1} s\right] P\right)\right)$ where $F=\prod_{j}(t-\psi([j s] P)) \in(\mathbf{Z} / n \mathbf{Z})[t]$. Standard fast-arithmetic algorithms perform this computation in time $(\#\{i\}+\#\{j\})^{1+o(1)}$ rather than $\#\{i\} \#\{j\}$ : first compute $F$ via a "product tree"; then compute the values $F\left(\psi\left(\left[i d_{1} s\right] P\right)\right)$ for all $i$ via a "remainder tree" or a "scaled remainder tree"; then multiply the values. For details and further speedups see, e.g., 6]; [47, Section 3]; and [7, Sections 12, 18].

5.4. Standard speedup: Higher-degree baby steps and giant steps. One can replace $[j s] P$ and $\left[i d_{1} s\right] P$ by, e.g., $\left[j^{6} s\right] P$ and $\left[\left(i d_{1}\right)^{6} s\right] P$. The advantage of this change is that one finds primes $\ell$ dividing $\left(i d_{1}\right)^{6} \pm j^{6}$, not just $i d_{1} \pm j$. If $i d_{1}$ and $j$ were uniformly distributed modulo $\ell$, then $\left(i d_{1}\right)^{6} \pm j^{6}$ would be more than twice as likely as $i d_{1} \pm j$ to be divisible by $\ell$. See [37, Section 5.3] for a probability analysis.

The only disadvantage is that there are more elliptic-curve operations. GMPECM computes $\left[j^{6} s\right] P$ for each integer $j \in\left\{1,2, \ldots, d_{1} / 2\right\}$ by computing the differences $\left[\left((j+1)^{6}-j^{6}\right) s\right] P$, the second differences $\left[\left((j+2)^{6}-2(j+1)^{6}+j^{6}\right) s\right] P$, etc.; the sixth differences are constants $[6 ! s] P$, so GMP-ECM uses a total of approximately $6\left(d_{1} / 2\right)$ elliptic-curve additions. More generally, GMP-ECM computes $\left[j^{e} s\right] P$ for each integer $j \in\left\{1,2, \ldots, d_{1} / 2\right\}$ using approximately $e\left(d_{1} / 2\right)$ ellipticcurve additions.

One consequence of this generalization is that elliptic-curve operations cannot be a negligible part of the time taken by a properly optimized stage 2 , compared to the time needed for computing the final product. If they were negligible, then increasing $e$ would find a considerable number of additional primes at negligible extra cost.

GMP-ECM actually uses $D_{e}(j)$ instead of $j^{e}$. Here $D_{e}$ is the degree-e "Dickson polynomial" defined by $D_{e}(t-1 / t)=t^{e}+(-1 / t)^{e}$. The differences $D_{e}\left(i d_{1}\right) \pm D_{e}(j)$ have the same chance as $\left(i d_{1}\right)^{e} \pm j^{e}$ to be divisible by $\ell$, but are less closely correlated than $\left(i d_{1}\right)^{e} \pm j^{e}$ as $(i, j)$ vary; see [37, Table 5.3.1].

5.5. The standard elliptic-curve coordinate system. GMP-ECM does not use Montgomery coordinates in stage 2. Montgomery coordinates allow efficient differential additions, but most of the additions involved in higher-degree steps are not differential additions: they are sums where the differences are unknown.

GMP-ECM instead switches to affine coordinates $(x, y)$. Addition in affine coordinates involves $1 \mathbf{I}+2 \mathbf{M}+1 \mathbf{S}$. For degree- $e$ steps there are $e$ additions to perform 
in parallel; GMP-ECM combines $e \mathbf{I}$ into $1 \mathbf{I}+3(e-1) \mathbf{M}$. GMP-ECM's total cost for baby steps is therefore $\left(d_{1} / 2\right) \mathbf{I}+(5 e-3)\left(d_{1} / 2\right) \mathbf{M}+e\left(d_{1} / 2\right) \mathbf{S}$.

5.6. Speedups in EECM-MPFQ. EECM-MPFQ uses higher-degree baby steps and giant steps, with the same Dickson polynomials $D_{e}$ used in GMP-ECM, but changes the elliptic-curve computations in three ways:

- EECM-MPFQ skips the $d_{1} / 2-\varphi\left(d_{1}\right) / 2$ values of $j \in\left\{1,2, \ldots, d_{1} / 2\right\}$ that have $\operatorname{gcd}\left\{j, d_{1}\right\}>1$. It computes $\left[D_{e}(j) s\right] P$ for the $\varphi\left(d_{1}\right) / 2$ values of $j$ that have $\operatorname{gcd}\left\{j, d_{1}\right\}=1$ (and $\left[D_{e}\left(i d_{1}\right) s\right] P$ for consecutive integers $i$ ). GMPECM tries to do something similar, focusing on the $d_{1} / 6$ values of $j$ in the arithmetic progression $(1+6 \mathbf{Z}) \cap\left[1, d_{1}\right]$; but $d_{1} / 6$ is considerably larger than $\varphi\left(d_{1}\right) / 2$.

- EECM-MPFQ delays all inversions until the elliptic-curve computations are finished. It computes the desired $y$-coordinates in one final batched division, costing $1 \mathbf{I}+(4(\#\{i\}+\#\{j\})-3) \mathbf{M}$ in total for the baby steps and the giant steps.

- EECM-MPFQ performs each intermediate elliptic-curve addition in extended Edwards coordinates, costing 9M. Occasionally an addition is a doubling costing only $3 \mathbf{M}+4 \mathbf{S}$.

EECM-MPFQ computes the desired multiples of $[s] P$ as a batch using a simple general-purpose multi-scalar-multiplication algorithm described in [22, Section 4] with credit to Bos and Coster. If $n_{1} \geq n_{2} \geq \cdots$, then the algorithm computes the multiples $\left[n_{1} s\right] P,\left[n_{2} s\right] P, \ldots$ by recursively computing $\left[\left(n_{1}-n_{2}\right) s\right] P,\left[n_{2} s\right] P, \ldots$ and then adding $\left[n_{2} s\right] P$ to $\left[\left(n_{1}-n_{2}\right) s\right] P$. Actually, the Bos-Coster algorithm recursively computes $\left[\left(n_{1} \bmod n_{2}\right) s\right] P,\left[n_{2} s\right] P, \ldots$ and then adds the appropriate multiple of $\left[n_{2} s\right] P$ to $\left[\left(n_{1} \bmod n_{2}\right) s\right] P$; but this refinement is irrelevant in the typical case that $n_{1}<2 n_{2}$.

Table 5.1 reports the number of multiplications used inside elliptic-curve operations in EECM-MPFQ's stage 2, for various choices of $d_{1}$ and $e$. The number of multiplications is divided by $\#\{i\}+\#\{j\}$ to produce each "cost" column. The final batched division costs 4 in this measure; the remaining cost is 9 times the per-output length of the Bos-Coster addition chain.

One can see from the table that the Bos-Coster addition chain has per-output length approximately 1 for $e=1 ; 1.9$ for $e=2 ; 3.3$ for $e=3$; 11 for $e=6$; and 22 for $e=12$. For comparison, the addition chain used in GMP-ECM has per-output length approximately $e d_{1} / \varphi\left(d_{1}\right)$ : i.e., roughly $4 e$ for the range of $d_{1}$ shown in the table. This does not imply that GMP-ECM would benefit from switching to the Bos-Coster addition chain: GMP-ECM's stage-2 time is determined not only by addition-chain length but also by the number of inversions that can be performed in parallel.

By default EECM-MPFQ uses MPFQ to compute the final product. However, the user can ask EECM-MPFQ to switch to product trees and scaled remainder trees, using Shoup's NTL library for fast polynomial arithmetic; this saves time when $\#\{i\}+\#\{j\}$ is sufficiently large. In theory, one can and should integrate these computations, using fast polynomial arithmetic to split the product computation into problems that are small enough to be handled efficiently by MPFQ; in practice, this approach is hampered by the difficulty of moving data between NTL and MPFQ. 
TABLE 5.1. Cost of elliptic-curve operations in stage 2 of EECM-MPFQ. Cost means the number of multiplications divided by $\#\{i\}+\#\{j\}$. Baby steps and giant steps are included. Multiplications used for inversion are included. Multiplications for the final product are not included.

\begin{tabular}{|c|c|c|c|c|c|c|c|c|c|}
\hline$d_{1}$ & $B_{1}$ & $\#\{j\}$ & $\#\{i\}$ & $d_{1} \#\{i\}$ & $\begin{array}{r}\text { Cost } \\
e=1\end{array}$ & $\begin{array}{r}\text { Cost } \\
e=2\end{array}$ & $\begin{array}{r}\text { Cost } \\
e=3\end{array}$ & $\begin{array}{r}\text { Cost } \\
e=6\end{array}$ & $\begin{array}{r}\text { Cost } \\
e=12\end{array}$ \\
\hline 30 & 60 & 4 & 4 & 120 & \begin{tabular}{|l|}
18.75000 \\
\end{tabular} & 38.00000 & 57.12500 & 114.50000 & 242.75000 \\
\hline 42 & 84 & 6 & 6 & 252 & 16.75000 & 33.41667 & 48.33333 & 106.16667 & 214.16667 \\
\hline 60 & 120 & 8 & 8 & 480 & 15.81250 & 30.56250 & 43.50000 & 104.25000 & 204.31250 \\
\hline 90 & 180 & 12 & 12 & 1080 & 14.87500 & 26.95833 & 41.95833 & 101.20833 & 205.83333 \\
\hline 120 & 240 & 16 & 16 & 1920 & 14.40625 & 26.56250 & 38.37500 & 98.56250 & 200.09375 \\
\hline 150 & 300 & 20 & 20 & 3000 & 14.12500 & 24.97500 & 39.57500 & 101.02500 & 201.15000 \\
\hline 180 & 360 & 24 & 24 & 4320 & 13.93750 & 24.47917 & 37.41667 & 100.04167 & 201.10417 \\
\hline 210 & 420 & 24 & 24 & 5040 & 14.33333 & 25.22917 & 39.10417 & 102.29167 & 205.41667 \\
\hline 330 & 660 & 40 & 40 & 13200 & 13.91250 & 23.60000 & 37.43750 & 102.35000 & 204.27500 \\
\hline 390 & 780 & 48 & 48 & 18720 & 13.65625 & 22.76042 & 36.64583 & 101.61458 & 202.30208 \\
\hline 420 & 840 & 48 & 48 & 20160 & 13.65625 & 22.66667 & 36.64583 & 102.64583 & 204.17708 \\
\hline 510 & 1020 & 64 & 64 & 32640 & 13.49219 & 22.42969 & 35.79688 & 101.04688 & 202.01563 \\
\hline 630 & 1260 & 72 & 72 & 45360 & 13.43750 & 22.31250 & 36.38889 & 101.57639 & 204.45139 \\
\hline 660 & 1320 & 80 & 80 & 52800 & 13.45625 & 22.06875 & 35.28750 & 100.76250 & 203.02500 \\
\hline 780 & 1560 & 96 & 96 & 74880 & 13.32813 & 21.63542 & 34.71354 & 100.94792 & 201.58854 \\
\hline 840 & 1680 & 96 & 96 & 80640 & 13.32813 & 22.05729 & 35.41667 & 99.11458 & 203.97917 \\
\hline 990 & 1980 & 120 & 120 & 118800 & 13.30417 & 21.78333 & 35.47083 & 100.45833 & 201.63333 \\
\hline 1050 & 2100 & 120 & 120 & 126000 & 13.26250 & 21.70417 & 35.80833 & 101.13333 & 203.32083 \\
\hline 1260 & 2520 & 144 & 144 & 181440 & 13.21875 & 21.63194 & 34.44444 & 100.85069 & 203.19444 \\
\hline 1470 & 2940 & 168 & 168 & 246960 & 13.18750 & 21.52381 & 34.43452 & 101.63988 & 202.43452 \\
\hline 1680 & 3360 & 192 & 192 & 322560 & 13.16406 & 21.48958 & 34.05208 & 97.09896 & 201.84115 \\
\hline 1890 & 3780 & 216 & 216 & 408240 & 13.14583 & 21.52315 & 34.37963 & 100.83796 & 201.42130 \\
\hline 2100 & 4200 & 240 & 240 & 504000 & 13.13125 & 21.42292 & 33.68542 & 100.58542 & 201.77917 \\
\hline 2310 & 4620 & 240 & 240 & 554400 & 13.18958 & 21.53542 & 34.43542 & 101.74792 & 203.80417 \\
\hline 2520 & 5040 & 288 & 288 & 725760 & 13.10938 & 21.23785 & 33.33160 & 97.80035 & 201.67535 \\
\hline 2730 & 5460 & 288 & 288 & 786240 & 13.15799 & 21.36285 & 33.78299 & 101.20660 & 202.51910 \\
\hline 2940 & 5880 & 336 & 336 & 987840 & 13.09375 & 21.11905 & 33.21280 & 99.97619 & 200.79762 \\
\hline 3150 & 6300 & 360 & 360 & 1134000 & 13.08750 & 21.16528 & 33.37639 & 100.29028 & 201.01528 \\
\hline 3360 & 6720 & 384 & 384 & 1290240 & 13.08203 & 21.12370 & 33.06510 & 96.26432 & 200.13932 \\
\hline 3570 & 7140 & 384 & 384 & 1370880 & 13.11719 & 21.27474 & 33.45052 & 100.72917 & 202.09635 \\
\hline 3780 & 7560 & 432 & 432 & 1632960 & 13.07292 & 21.16898 & 33.15856 & 99.90856 & 200.72107 \\
\hline 3990 & 7980 & 432 & 432 & 1723680 & 13.10532 & 21.34606 & 33.53356 & 100.72106 & 201.30440 \\
\hline 4200 & 8400 & 480 & 480 & 2016000 & 13.06563 & 21.17708 & 33.02708 & 97.48021 & 200.33333 \\
\hline 4290 & 8580 & 480 & 480 & 2059200 & 13.09479 & 20.99896 & 33.44896 & 100.30208 & 200.83958 \\
\hline 4620 & 9240 & 480 & 480 & 2217600 & 13.09479 & 21.32708 & 33.43021 & 100.70521 & 202.04896 \\
\hline
\end{tabular}

5.7. Measurements of CPU cycles. A 300-curve test of EECM-MPFQ took 4.7 million cycles per curve on a single core of a $3.2 \mathrm{GHz}$ AMD Phenom II X4 (100f42) for a 240-bit $n$ with $B_{1}=1024, d_{1}=630, \#\{i\}=72, \#\{j\}=72$, and $e=1$. Here $B_{1}+d_{1} \#\{i\}=46384$. For comparison, a 300-curve test of GMP-ECM took 10.8 million cycles per curve on the same CPU for the same 240-bit $n$ with $B_{1}=1024, B_{2}=41526$, and $e=1$. GMP-ECM took 11.5 million cycles per curve with $B_{2}=50646$, the next $B_{2}$ supported by GMP-ECM after 41526 . 
Increasing $e$ to 3 increased the EECM-MPFQ time with $B_{1}+d_{1} \#\{i\}=46384$ to 5.4 million cycles per curve, increased the GMP-ECM time with $B_{2}=41526$ to 13.4 million cycles per curve, and increased the GMP-ECM time with $B_{2}=50646$ to 14.7 million cycles per curve.

Increasing $d_{1}$ to 510510, \#\{i\} to 46080, and $e$ to 12 increased the EECM-MPFQ time to 34000 million cycles per curve. Here $d_{1} \#\{i\}=23524300800$. For comparison, GMP-ECM took only 18000 million cycles per curve with $e=12$ and $B_{2}=23412731170$; for such large sizes GMP-ECM includes, and benefits from, FFT-based-polynomial-arithmetic optimizations not present in NTL and consequently not present in EECM-MPFQ.

\section{EdWARDS CURVES With LARGE TORSION}

Mazur's theorem [34, Theorem 2] says that the torsion group $E_{\text {tor }}(\mathbf{Q})$ of any elliptic curve $E$ is isomorphic to one of 15 finite groups: specifically,

$$
E_{\text {tor }}(\mathbf{Q}) \cong\left\{\begin{array}{lll}
\mathbf{Z} / m \mathbf{Z}, & m \in\{1,2,3,4,5,6,7,8,9,10,12\}, \\
\mathbf{Z} / 2 \mathbf{Z} \times \mathbf{Z} / 2 m \mathbf{Z}, & m \in\{1,2,3,4\} .
\end{array}\right.
$$

Any Edwards curve has a point $(1,0)$ of order 4 . It follows that the torsion group of an Edwards curve is isomorphic to either $\mathbf{Z} / 4 \mathbf{Z}, \mathbf{Z} / 8 \mathbf{Z}, \mathbf{Z} / 12 \mathbf{Z}, \mathbf{Z} / 2 \mathbf{Z} \times \mathbf{Z} / 4 \mathbf{Z}$, or $\mathbf{Z} / 2 \mathbf{Z} \times \mathbf{Z} / 8 \mathbf{Z}$.

The most interesting cases for ECM are $\mathbf{Z} / 12 \mathbf{Z}$ and $\mathbf{Z} / 2 \mathbf{Z} \times \mathbf{Z} / 8 \mathbf{Z}$, since they force the group orders of $E$ modulo primes $p$ (of good reduction) to be divisible by 12 and 16 respectively. In this section we show which conditions an Edwards curve $x^{2}+y^{2}=1+d x^{2} y^{2}$ over $\mathbf{Q}$ must satisfy to have torsion group isomorphic to $\mathbf{Z} / 12 \mathbf{Z}$ or $\mathbf{Z} / 2 \mathbf{Z} \times \mathbf{Z} / 8 \mathbf{Z}$. We give parameterizations for both cases.

One could hope to force divisibility by 12 in a different way, namely by finding a twisted Edwards curve with $\mathbf{Q}$-torsion group isomorphic to $\mathbf{Z} / 2 \mathbf{Z} \times \mathbf{Z} / 6 \mathbf{Z}$. A twisted Edwards curve does not need to have a point of order 4. However, we will show that there are no twisted Edwards curves with Q-torsion group isomorphic to $\mathbf{Z} / 2 \mathbf{Z} \times \mathbf{Z} / 6 \mathbf{Z}$. We will also show that there are no twisted Edwards curves with $\mathbf{Q}$-torsion group isomorphic to $\mathbf{Z} / 10 \mathbf{Z}$.

Computations in extended Edwards coordinates would benefit from using twisted Edwards curves with $a=-1$. We show that such curves cannot have $\mathbf{Q}$-torsion group isomorphic to $\mathbf{Z} / 12 \mathbf{Z}$ or $\mathbf{Z} / 2 \mathbf{Z} \times \mathbf{Z} / 8 \mathbf{Z}$.

We first present the constructions and then show the impossibility results.

6.1. Torsion group $\mathbf{Z} / 12 \mathbf{Z}$. Theorem 6.2 states a genus-0 cover of the set of Edwards curves over $\mathbf{Q}$ with torsion group $\mathbf{Z} / 12 \mathbf{Z}$. Theorem 6.3 identifies all the points of finite order on such curves. Theorem 6.4 states a rational cover.

Theorem 6.2. If $y_{3} \in \mathbf{Q} \backslash\{-2,-1 / 2,0, \pm 1\}$ and $x_{3} \in \mathbf{Q}$ satisfy the equation $x_{3}^{2}=-\left(y_{3}^{2}+2 y_{3}\right)$, then the Edwards curve $x^{2}+y^{2}=1+d x^{2} y^{2}$ over $\mathbf{Q}$, where $d=-\left(2 y_{3}+1\right) /\left(x_{3}^{2} y_{3}^{2}\right)$, has $\left(x_{3}, y_{3}\right)$ as a point of order 3 and has $\mathbf{Q}$-torsion group isomorphic to $\mathbf{Z} / 12 \mathbf{Z}$. Conversely, every Edwards curve over $\mathbf{Q}$ with a point of order 3 arises in this way.

Proof. Assume that such a $y_{3}$ and $x_{3}$ exist. Then $d$ is defined and not equal to 0 or 1 , and $x_{3}^{2}+y_{3}^{2}=-2 y_{3}=1+d x_{3}^{2} y_{3}^{2}$. By Theorem 3.3. $\left(x_{3}, y_{3}\right)$ is a point of order 3 on $\overline{\mathrm{E}}_{\mathrm{E}, 1, d}(\mathbf{Q})$. Since each Edwards curve has a point of order 4 the torsion group 
must contain a copy of $\mathbf{Z} / 12 \mathbf{Z}$. By Mazur's theorem the torsion group cannot be larger.

Conversely, if $\overline{\mathrm{E}}_{\mathrm{E}, 1, d}(\mathbf{Q})$ has a point of order 3, then by Theorem 3.3 the point can be written as $\left(x_{3}, y_{3}\right)$ where $x_{3}^{2}+y_{3}^{2}=1+d x_{3}^{2} y_{3}^{2}=-2 y_{3}$. Hence $x_{3}^{2}=-\left(y_{3}^{2}+2 y_{3}\right)$. Note that $x_{3} \neq 0$, since otherwise $y_{3}^{2}=1=-2 y_{3}$; and note that $y_{3} \notin\{0,-2\}$ since otherwise $x_{3}=0$. Now $d=-\left(2 y_{3}+1\right) /\left(x_{3}^{2} y_{3}^{2}\right)$. Finally, note that $y_{3} \notin\{-1 / 2, \pm 1\}$ since otherwise $d \in\{0,1\}$, contradicting the definition of an Edwards curve.

Theorem 6.3. Let $x^{2}+y^{2}=1+d x^{2} y^{2}$ be an Edwards curve over $\mathbf{Q}$ with $E_{\mathrm{tor}}(\mathbf{Q}) \cong$ $\mathbf{Z} / 12 \mathbf{Z}$ and let $P_{3}=\left(x_{3}, y_{3}\right)$ be a point of order 3 on the curve.

The 12 torsion points on the curve and their respective orders are as follows:

$$
\begin{array}{c||c|c|c|c|c|c}
\text { point } & (0,1) & (0,-1) & \left( \pm x_{3}, y_{3}\right) & ( \pm 1,0) & \left( \pm x_{3},-y_{3}\right) & \left( \pm y_{3}, \pm x_{3}\right) \\
\hline \text { order } & 1 & 2 & 3 & 4 & 6 & 12
\end{array}
$$

Proof. The points of order 6 are obtained as $\left( \pm x_{3}, y_{3}\right)+(0,-1)$, the points of order 12 by adding $( \pm 1,0)$ to the points of order 3 .

Theorem 6.4. If $u \in \mathbf{Q} \backslash\{0, \pm 1\}$, then the Edwards curve $x^{2}+y^{2}=1+d x^{2} y^{2}$ over $\mathbf{Q}$, where

$$
x_{3}=\frac{u^{2}-1}{u^{2}+1}, \quad y_{3}=-\frac{(u-1)^{2}}{u^{2}+1}, \quad d=\frac{\left(u^{2}+1\right)^{3}\left(u^{2}-4 u+1\right)}{(u-1)^{6}(u+1)^{2}}
$$

has $\left(x_{3}, y_{3}\right)$ as a point of order 3 and has $\mathbf{Q}$-torsion group isomorphic to $\mathbf{Z} / 12 \mathbf{Z}$. Conversely, every Edwards curve over $\mathbf{Q}$ with a point of order 3 arises in this way.

The parameters $u$ and $1 / u$ give the same value of $d$.

Proof. Multiply the identity $(u+1)^{2}+(u-1)^{2}=2\left(u^{2}+1\right)$ by $(u-1)^{2} /\left(u^{2}+1\right)^{2}$ to see that $x_{3}^{2}+y_{3}^{2}=-2 y_{3}$, and observe that

$$
d=\frac{2(u-1)^{2}-\left(u^{2}+1\right)}{u^{2}+1} \cdot \frac{\left(u^{2}+1\right)^{2}}{\left(u^{2}-1\right)^{2}} \cdot \frac{\left(u^{2}+1\right)^{2}}{(u-1)^{4}}=\frac{-2 y_{3}-1}{x_{3}^{2} y_{3}^{2}} .
$$

Furthermore, $y_{3} \notin\{-2,-1 / 2,0, \pm 1\}$ since $u \in \mathbf{Q} \backslash\{0, \pm 1\}$. By Theorem 6.2, the Edwards curve $x^{2}+y^{2}=1+d x^{2} y^{2}$ over $\mathbf{Q}$ has $\left(x_{3}, y_{3}\right)$ as a point of order 3 and has torsion group isomorphic to $\mathbf{Z} / 12 \mathbf{Z}$.

Conversely, assume that the Edwards curve $x^{2}+y^{2}=1+d x^{2} y^{2}$ has a point of order 3. By Theorem 6.2, the curve has a point $\left(x_{3}, y_{3}\right)$ of order 3 for some $y_{3} \in \mathbf{Q} \backslash\{-2,-1 / 2,0, \pm 1\}$ and $x_{3} \in \mathbf{Q}$ satisfying $x_{3}^{2}=-\left(y_{3}^{2}+2 y_{3}\right)$ and $d=$ $-\left(2 y_{3}+1\right) /\left(x_{3}^{2} y_{3}^{2}\right)$. Note that $\left(x_{3}, y_{3}+1\right)$ is a point on the unit circle.

If $x_{3}= \pm 1$, then $y_{3}+1=0$ so $d=-\left(2 y_{3}+1\right) /\left(x_{3}^{2} y_{3}^{2}\right)=1$; but Edwards curves have $d \neq 1$. Hence $x_{3} \neq \pm 1$. Furthermore, $x_{3} \neq 0$ since every point with $x$-coordinate 0 has order 1 or 2 .

Define $u$ as the slope of the line between $(1,0)$ and $\left(x_{3},-\left(y_{3}+1\right)\right)$ : i.e., $u=$ $\left(y_{3}+1\right) /\left(1-x_{3}\right)$. Substitute $y_{3}+1=u\left(1-x_{3}\right)$ into $\left(y_{3}+1\right)^{2}=1-x_{3}^{2}$ to obtain $u^{2}\left(1-x_{3}\right)^{2}=1-x_{3}^{2}=\left(1+x_{3}\right)\left(1-x_{3}\right)$, i.e., $u^{2}\left(1-x_{3}\right)=1+x_{3}$, i.e., $x_{3}=\left(u^{2}-1\right) /\left(u^{2}+1\right)$. Then $u \notin\{0, \pm 1\}$ since $x_{3} \notin\{0,-1\}$. Furthermore, $y_{3}=u\left(1-x_{3}\right)-1=u\left(2 /\left(u^{2}+1\right)\right)-1=-(u-1)^{2} /\left(u^{2}+1\right)$ and as above $d=\left(2 y_{3}+1\right) /\left(x_{3}^{2} y_{3}^{2}\right)=\left(u^{2}+1\right)^{3}\left(u^{2}-4 u+1\right) /\left((u-1)^{6}(u+1)^{2}\right)$.

The value of $d$ is invariant under the change $u \mapsto 1 / u$ since

$$
\frac{\left(1+u^{2}\right)^{3}\left(1-4 u+u^{2}\right)}{(1-u)^{6}(1+u)^{2}}=\frac{\left(u^{2}+1\right)^{3}\left(u^{2}-4 u+1\right)}{(u-1)^{6}(u+1)^{2}} .
$$


Solving the equation $d\left(u^{\prime}\right)=d(u)$ for $u^{\prime}$ in terms of $u$ over the rationals shows that $u \mapsto 1 / u$ is the only rational transformation leaving $d$ invariant that works independently of $u$.

6.5. Torsion group $\mathbf{Z} / 2 \mathbf{Z} \times \mathbf{Z} / 8 \mathbf{Z}$. Theorem 6.6 states a genus- 0 cover of the set of Edwards curves over $\mathbf{Q}$ with torsion group $\mathbf{Z} / 2 \mathbf{Z} \times \mathbf{Z} / 8 \mathbf{Z}$. Theorem 6.8 identifies all the affine points of finite order on such curves. Theorem 6.9 states a rational cover and identifies the degree of the cover.

There are actually two types of curves in Theorem 6.6 points of order 8 double to $( \pm 1,0)$ on curves of the first type, or to $((1: \pm \sqrt{d}),(1: 0))$ on curves of the second type. Curves of the second type are birationally equivalent to curves of the first type by Theorem 6.7. Subsequent theorems consider only the first type.

Theorem 6.6. If $x_{8} \in \mathbf{Q} \backslash\{0, \pm 1\}$ and $d=\left(2 x_{8}^{2}-1\right) / x_{8}^{4}$ is a square in $\mathbf{Q}$, then the Edwards curve $x^{2}+y^{2}=1+d x^{2} y^{2}$ over $\mathbf{Q}$ has $\left(x_{8}, \pm x_{8}\right)$ as points of order 8 doubling to $( \pm 1,0)$, and has $\mathbf{Q}$-torsion group isomorphic to $\mathbf{Z} / 2 \mathbf{Z} \times \mathbf{Z} / 8 \mathbf{Z}$. Conversely, every Edwards curve over $\mathbf{Q}$ with $\mathbf{Q}$-torsion group isomorphic to $\mathbf{Z} / 2 \mathbf{Z} \times \mathbf{Z} / 8 \mathbf{Z}$ and a point of order 8 doubling to $( \pm 1,0)$ arises in this way.

If $\bar{x}_{8} \in \mathbf{Q} \backslash\{0, \pm 1\}$ and $d=1 /\left(\bar{x}_{8}^{2}\left(2-\bar{x}_{8}^{2}\right)\right)$ is a square in $\mathbf{Q}$, then the Edwards curve $x^{2}+y^{2}=1+d x^{2} y^{2}$ over $\mathbf{Q}$ has $\left(\bar{x}_{8}, \pm 1 /\left(\bar{x}_{8} \sqrt{d}\right)\right)$ as points of order 8 doubling to $((1: \pm \sqrt{d}),(1: 0))$, and has $\mathbf{Q}$-torsion group isomorphic to $\mathbf{Z} / 2 \mathbf{Z} \times \mathbf{Z} / 8 \mathbf{Z}$. Conversely, every Edwards curve over $\mathbf{Q}$ with $\mathbf{Q}$-torsion group isomorphic to $\mathbf{Z} / 2 \mathbf{Z} \times \mathbf{Z} / 8 \mathbf{Z}$ and a point of order 8 doubling to $((1: \pm \sqrt{d}),(1: 0))$ arises in this way.

Every Edwards curve over $\mathbf{Q}$ with $\mathbf{Q}$-torsion group isomorphic to $\mathbf{Z} / 2 \mathbf{Z} \times \mathbf{Z} / 8 \mathbf{Z}$ arises in one of these two ways.

Proof. Any such $x_{8}$ yields $d \neq 0,1$, so $x^{2}+y^{2}=1+d x^{2} y^{2}$ is an Edwards curve. By Theorems 3.1 and 3.2 the curve has points $(0,-1)$ and $((1: 0),(1: \pm \sqrt{d}))$ of order 2 , and points $\left(x_{8}, \pm x_{8}\right)$ of order 8 doubling to $( \pm 1,0)$. Similarly, any such $\bar{x}_{8}$ yields an Edwards curve with points $(0,-1)$ and $((1: 0),(1: \pm \sqrt{d}))$ of order 2 and $\left(\bar{x}_{8}, \pm 1 /\left(\bar{x}_{8} \sqrt{d}\right)\right)$ of order 8 doubling to $((1: \pm \sqrt{d}),(1: 0))$.

In both cases the torsion group contains a copy of $\mathbf{Z} / 2 \mathbf{Z} \times \mathbf{Z} / 8 \mathbf{Z}$. By Mazur's theorem the torsion group cannot be larger.

Conversely, assume that $x^{2}+y^{2}=1+d x^{2} y^{2}$ is an Edwards curve with Qtorsion group isomorphic to $\mathbf{Z} / 2 \mathbf{Z} \times \mathbf{Z} / 8 \mathbf{Z}$. There are four elements of order 4 in $\mathbf{Z} / 2 \mathbf{Z} \times \mathbf{Z} / 8 \mathbf{Z}$, all doubling to the same element, so there are four order- 4 points on the curve, all doubling to the same point.

The points $( \pm 1,0)$ have order 4 and double to $(0,-1)$, so the other two points of order 4 also double to $(0,-1)$. By Theorem 3.1. those other two points must be $((1: \pm \sqrt{d}),(1: 0))$, and $d$ must be a square.

Now any point of order 8 must double to $( \pm 1,0)$ or to $((1: \pm \sqrt{d}),(1: 0))$. In the first case, by Theorem 3.2 , the point is $\left(x_{8}, \pm x_{8}\right)$ for some root $x_{8}$ of $d x_{8}^{4}-2 x_{8}^{2}+1$; hence $x_{8} \notin\{0, \pm 1\}$ and $d=\left(2 x_{8}^{2}-1\right) / x_{8}^{4}$. In the second case, by Theorem 3.2. the point is $\left(\bar{x}_{8}, \pm 1 /\left(\bar{x}_{8} \sqrt{d}\right)\right)$ for some root $\bar{x}_{8}$ of $d \bar{x}_{8}^{4}-2 d \bar{x}_{8}^{2}+1$; hence $\bar{x}_{8} \notin\{0, \pm 1\}$ and $d=1 /\left(\bar{x}_{8}^{4}-2 \bar{x}_{8}^{2}\right)$.

Theorem 6.7. Let $d$ be a square. The Edwards curves $x^{2}+y^{2}=1+d x^{2} y^{2}$ and $\bar{x}^{2}+\bar{y}^{2}=1+(1 / d) \bar{x}^{2} \bar{y}^{2}$ are birationally equivalent via the map $\bar{x}=x \sqrt{d}, \bar{y}=1 / y$ with inverse $x=\bar{x} / \sqrt{d}, y=1 / \bar{y}$. The map fixes $(0, \pm 1)$. 
Proof. Inserting $\bar{x}=x \sqrt{d}, \bar{y}=1 / y$ into $x^{2}+y^{2}=1+d x^{2} y^{2}$ gives $\bar{x}^{2} / d+1 / \bar{y}^{2}=$ $1+\bar{x}^{2} / \bar{y}^{2}$ which after multiplication by $\bar{y}^{2}$ gives $\bar{x}^{2} \bar{y}^{2} / d+1=\bar{y}^{2}+\bar{x}^{2}$. The only exceptional points are $( \pm 1,0)$. The statement about $(0, \pm 1)$ follows by direct inspection.

In particular, each curve of the second type in Theorem 6.6 is birationally equivalent to a curve of the first type. Indeed, assume that $\bar{x}_{8} \in \mathbf{Q} \backslash\{0, \pm 1\}$ and that $d=1 /\left(\bar{x}_{8}^{2}\left(2-\bar{x}_{8}^{2}\right)\right)$ is a square in $\mathbf{Q}$. Define $x_{8}=\bar{x}_{8} \sqrt{d}$. Then $x_{8}^{2}=1 /\left(2-\bar{x}_{8}^{2}\right)$, so $\left(2 x_{8}^{2}-1\right) / x_{8}^{4}=\left(2 /\left(2-\bar{x}_{8}^{2}\right)-1\right)\left(2-\bar{x}_{8}^{2}\right)^{2}=\bar{x}_{8}^{2}\left(2-\bar{x}_{8}^{2}\right)=1 / d$, which is a square; furthermore, $x_{8} \notin\{0, \pm 1\}$ since $2-\bar{x}_{8}^{2} \neq 1$ since $\bar{x}_{8} \notin\{ \pm 1\}$. Hence $x^{2}+y^{2}=1+(1 / d) x^{2} y^{2}$ is a curve of the first type. The curve $x^{2}+y^{2}=1+d x^{2} y^{2}$ is birationally equivalent to $\bar{x}^{2}+\bar{y}^{2}=1+(1 / d) \bar{x}^{2} \bar{y}^{2}$ by Theorem 6.7. Consequently, we can restrict attention to curves of the first type, i.e., curves on which the points of order 8 double to $( \pm 1,0)$.

Theorem 6.8. Assume that $x_{8} \in \mathbf{Q} \backslash\{0, \pm 1\}$ and that $d=\left(2 x_{8}^{2}-1\right) / x_{8}^{4}$ is a square in $\mathbf{Q}$. Then there are 16 points of finite order on $\overline{\mathrm{E}}_{\mathrm{E}, 1, d}$ over $\mathbf{Q}$. The affine points of finite order are as follows:

$$
\begin{array}{c||c|c|c|c|c}
\text { point } & (0,1) & (0,-1) & ( \pm 1,0) & \left( \pm x_{8}, \pm x_{8}\right) & \left( \pm 1 /\left(x_{8} \sqrt{d}\right), \pm 1 /\left(x_{8} \sqrt{d}\right)\right) \\
\hline \text { order } & 1 & 2 & 4 & 8 & 8
\end{array}
$$

where the signs are taken independently.

Proof. Theorem 3.1 (with $a=1$ ) shows that the 4 affine points $(0,1),(0,-1)$, and $( \pm 1,0)$ are on $\overline{\mathrm{E}}_{\mathrm{E}, 1, d}$ and have the stated orders. It also shows that the 2 nonaffine points $((1: 0),(1: \pm \sqrt{d}))$ have order 2 and that the 2 nonaffine points $((1: \pm \sqrt{d}),(1: 0))$ have order 4 . Theorem 3.2 shows that the other affine points listed are 8 distinct points on $\overline{\mathrm{E}}_{\mathrm{E}, 1, d}$ and have order 8 . The torsion group has exactly 16 elements by Theorem 6.6.

Theorem 6.9. If $u \in \mathbf{Q} \backslash\{0,-1,-2\}$, then the Edwards curve $x^{2}+y^{2}=1+d x^{2} y^{2}$ over $\mathbf{Q}$, where

$$
x_{8}=\frac{u^{2}+2 u+2}{u^{2}-2}, \quad d=\frac{2 x_{8}^{2}-1}{x_{8}^{4}},
$$

has $\left(x_{8}, x_{8}\right)$ as a point of order 8 and has $\mathbf{Q}$-torsion group isomorphic to $\mathbf{Z} / 2 \mathbf{Z} \times$ $\mathbf{Z} / 8 \mathbf{Z}$.

Conversely, every Edwards curve over $\mathbf{Q}$ with torsion group isomorphic to $\mathbf{Z} / 2 \mathbf{Z} \times \mathbf{Z} / 8 \mathbf{Z}$ on which the points of order 8 double to $( \pm 1,0)$ is expressible in this way.

The parameters $u, 2 / u,-2(u+1) /(u+2),-(2+u) /(1+u),-(u+2),-2 /(u+2)$, $-u /(u+1)$, and $-2(u+1) / u$ give the same value of $d$ and they are the only values giving this $d$.

Proof. Divide the identity $2\left(u^{2}+2 u+2\right)^{2}-\left(u^{2}-2\right)^{2}=\left(u^{2}+4 u+2\right)^{2}$ by $\left(u^{2}-2\right)^{2}$ to see that $2 x_{8}^{2}-1=\left(u^{2}+4 u+2\right)^{2} /\left(u^{2}-2\right)^{2}$. Hence $d$ is a square. Furthermore, $x_{8} \neq 0$ since $u^{2}+2 u+2 \neq 0 ; x_{8} \neq 1$ since $u \neq-2$; and $x_{8} \neq-1$ since $u \notin\{0,-1\}$. By Theorem 6.6] the curve $\overline{\mathrm{E}}_{\mathrm{E}, 1, d}$ has $\left(x_{8}, x_{8}\right)$ as a point of order 8, and has Q-torsion group isomorphic to $\mathbf{Z} / 2 \mathbf{Z} \times \mathbf{Z} / 8 \mathbf{Z}$.

Conversely, assume that an Edwards curve has torsion group isomorphic to $\mathbf{Z} / 2 \mathbf{Z} \times \mathbf{Z} / 8 \mathbf{Z}$ and has a point of order 8 doubling to $( \pm 1,0)$. By Theorem 6.6 , the 
curve can be expressed as $\overline{\mathrm{E}}_{\mathrm{E}, 1, d}$ for some $x_{8} \in \mathbf{Q} \backslash\{0, \pm 1\}$ such that $d=\left(2 x_{8}^{2}-1\right) / x_{8}^{4}$ is a square in $\mathbf{Q}$; i.e., such that $2 x_{8}^{2}-1$ is a square in $\mathbf{Q}$.

Choose $r \in \mathbf{Q}$ such that $2 x_{8}^{2}-1=r^{2}$. Define $u$ as the slope of the line between $(1,-1)$ and $\left(x_{8}, r\right)$ : i.e., $u=(r+1) /\left(x_{8}-1\right)$. Substitute $r=u\left(x_{8}-1\right)-1$ into $2\left(x_{8}^{2}-1\right)=(r+1)(r-1)$ to obtain $2\left(x_{8}^{2}-1\right)=u\left(x_{8}-1\right)\left(u\left(x_{8}-1\right)-2\right)$, i.e., $2\left(x_{8}+1\right)=$ $u\left(u\left(x_{8}-1\right)-2\right)$, i.e., $2 x_{8}+2=u^{2} x_{8}-u^{2}-2 u$; i.e., $x_{8}=\left(u^{2}+2 u+2\right) /\left(u^{2}-2\right)$. Finally, $u \notin\{0,-1\}$ since $x_{8} \neq-1$, and $u \neq-2$ since $x_{8} \neq 1$.

The identity

$$
\begin{aligned}
&(d(u)-d(v))\left((u+1)^{2}+1\right)^{4}\left((v+1)^{2}+1\right)^{4} \\
&=16(u-v)(u v-2)((u+2) v+2(u+1))(u+2+(u+1) v) \\
& \cdot(u+v+2)((u+2) v+2)(u+(u+1) v)(u v+2(u+1))
\end{aligned}
$$

immediately shows that if $v$ is any of the listed values $u, 2 / u, \ldots$ then $d(v)=d(u)$. Conversely, if $v$ is not one of those values then none of the factors $u-v, u v-2, \ldots$ are 0 so $d(v) \neq d(u)$.

6.10. Impossibility results. The following theorem shows that the only way for a twisted Edwards curve to have exactly 12 torsion points is to have torsion group isomorphic to $\mathbf{Z} / 12 \mathbf{Z}$. The next two theorems consider twisted Edwards curves with $a=-1$ and show that these cannot have $\mathbf{Q}$-torsion group isomorphic to $\mathbf{Z} / 12 \mathbf{Z}$ or $\mathbf{Z} / 2 \mathbf{Z} \times \mathbf{Z} / 8 \mathbf{Z}$. The last theorem shows that a twisted Edwards curve cannot have exactly 10 torsion points.

Theorem 6.11. There exists no twisted Edwards curve over $\mathbf{Q}$ with torsion group isomorphic to $\mathbf{Z} / 2 \mathbf{Z} \times \mathbf{Z} / 6 \mathbf{Z}$.

Proof. Let $a, d$ be distinct nonzero elements of $\mathbf{Q}$. Suppose that the twisted Edwards curve $\overline{\mathrm{E}}_{\mathrm{E}, a, d}: a x^{2}+y^{2}=1+d x^{2} y^{2}$ has $\mathbf{Q}$-torsion group isomorphic to $\mathbf{Z} / 2 \mathbf{Z} \times \mathbf{Z} / 6 \mathbf{Z}$.

There are three elements of order 2 in $\mathbf{Z} / 2 \mathbf{Z} \times \mathbf{Z} / 6 \mathbf{Z}$, so there are three points of order 2 in $\overline{\mathrm{E}}_{\mathrm{E}, a, d}(\mathbf{Q})$. By Theorem 3.1 the only possible points of order 2 are $(0,-1)$ and $((1: 0),( \pm \sqrt{a / d}: 1))$. Hence $\sqrt{a / d} \in \mathbf{Q}$.

There are also elements of order 3 in $\mathbf{Z} / 2 \mathbf{Z} \times \mathbf{Z} / 6 \mathbf{Z}$. Choose a point of order 3 in $\overline{\mathrm{E}}_{\mathrm{E}, a, d}(\mathbf{Q})$. By Theorem 3.3 this point can be expressed as $\left(x_{3}, y_{3}\right)$ where $a x_{3}^{2}+y_{3}^{2}=1+d x_{3}^{2} y_{3}^{2}=-2 y_{3}$.

Write $u=1+y_{3}$. Then $1-u^{2}=-2 y_{3}-y_{3}^{2}=a x_{3}^{2}$. Starting from $d x_{3}^{2} y_{3}^{2}=$ $a x_{3}^{2}+y_{3}^{2}-1$, replace $x_{3}^{2}$ by $\left(1-u^{2}\right) / a$ and replace $y_{3}$ by $u-1$ to see that $(d / a)(1-$ $\left.u^{2}\right)(u-1)^{2}=\left(1-u^{2}\right)+(u-1)^{2}-1=1-2 u$. Hence $s^{2}=4(1-2 u)\left(1-u^{2}\right)$ where $s=2\left(1-u^{2}\right)(u-1) \sqrt{d / a} \in \mathbf{Q}$.

In other words, $(2 u, s)$ is a $\mathbf{Q}$-rational point $(\sigma, \tau)$ on the elliptic curve $\tau^{2}=$ $\sigma^{3}-\sigma^{2}-4 \sigma+4$. This elliptic curve has rank 0 over $\mathbf{Q}$, and has exactly 7 affine points over $\mathbf{Q}$, as one can verify by typing

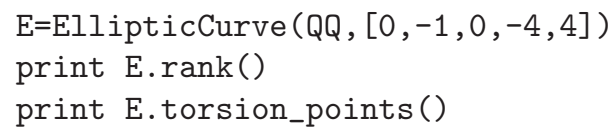

into the Sage computer-algebra system [43]. Specifically, $(\sigma, \tau)$ must be one of $( \pm 2,0),(0, \pm 2),(1,0),(4, \pm 6)$. Hence $u \in\{ \pm 1,0,1 / 2,2\}$. In each case $(a: d)=$ $\left(\left(1-u^{2}\right)(u-1)^{2}: 1-2 u\right) \in\{(1: 1),(0: 1),(1: 0)\}$, contradicting the assumption that $a, d$ are distinct nonzero elements of $\mathbf{Q}$. 
Theorem 6.12. There exists no twisted Edwards curve of the form $a x^{2}+y^{2}=$ $1+d x^{2} y^{2}$ over $\mathbf{Q}$ with $a=-1$ and torsion group isomorphic to $\mathbf{Z} / 12 \mathbf{Z}$.

Proof. Suppose that the twisted Edwards curve $\overline{\mathrm{E}}_{\mathrm{E},-1, d}:-x^{2}+y^{2}=1+d x^{2} y^{2}$ has Q-torsion group isomorphic to $\mathbf{Z} / 12 \mathbf{Z}$.

There is a unique element of order 2 in $\mathbf{Z} / 12 \mathbf{Z}$, so $(0,-1)$ is the only point of order 2 on $\overline{\mathrm{E}}_{\mathrm{E},-1, d}(\mathbf{Q})$. Furthermore, there are elements of order 4 in $\mathbf{Z} / 12 \mathbf{Z}$, so there are points on $\overline{\mathrm{E}}_{\mathrm{E},-1, d}(\mathbf{Q})$ doubling to $(0,-1)$. By Theorem 3.1 the only possibilities for such points are $((1: \pm \sqrt{a}),(0: 1))$ or $((1: \pm \sqrt{d}),(1: 0))$. Hence $a$ or $d$ is a square in $\mathbf{Q}$; but $a=-1$ is not a square in $\mathbf{Q}$, so $d$ is a square in $\mathbf{Q}$.

There are also elements of order 3 in $\mathbf{Z} / 12 \mathbf{Z}$. As in the proof of Theorem 6.11 there exists $u \in \mathbf{Q}$ such that $(d / a)\left(1-u^{2}\right)(u-1)^{2}=1-2 u$. Here $a=-1$ so $s^{2}=-4\left(1-u^{2}\right)(1-2 u)$ where $s=2\left(1-u^{2}\right)(u-1) \sqrt{d} \in \mathbf{Q}$.

In other words, $(-2 u, s)$ is a Q-rational point on the elliptic curve $\tau^{2}=\sigma^{3}+$ $\sigma^{2}-4 \sigma-4$. This elliptic curve has rank 0 over $\mathbf{Q}$, and has exactly 3 affine points over Q: specifically, $(\sigma, \tau)$ must be one of $( \pm 2,0),(-1,0)$. Hence $u \in\{ \pm 1,1 / 2\}$. If $u \in\{ \pm 1\}$ then $0=(d / a)\left(1-u^{2}\right)(u-1)^{2}=1-2 u \neq 0$, contradiction; if $u=1 / 2$ then $0=1-2 u=(d / a)\left(1-u^{2}\right)(u-1)^{2} \neq 0$, contradiction.

Theorem 6.13. There exists no twisted Edwards curve of the form $a x^{2}+y^{2}=$ $1+d x^{2} y^{2}$ over $\mathbf{Q}$ with $a=-1$ and torsion group isomorphic to $\mathbf{Z} / 2 \mathbf{Z} \times \mathbf{Z} / 8 \mathbf{Z}$.

Proof. Suppose that the twisted Edwards curve $\overline{\mathrm{E}}_{\mathrm{E},-1, d}:-x^{2}+y^{2}=1+d x^{2} y^{2}$ has Q-torsion group isomorphic to $\mathbf{Z} / 2 \mathbf{Z} \times \mathbf{Z} / 8 \mathbf{Z}$.

The torsion group contains exactly three elements of order 2 , so $\sqrt{a / d} \in \mathbf{Q}$ as in the proof of Theorem 6.11 i.e., $\sqrt{-d} \in \mathbf{Q}$. Consequently, $d$ is not a square in $\mathbf{Q}$.

The torsion group also contains exactly 4 elements of order 4 . These elements cannot double to $(0,-1)$ : otherwise they would have the form $((1: \pm \sqrt{-1}),(0: 1))$ or $((1: \pm \sqrt{d}),(1: 0))$ by Theorem 3.1 , but neither -1 nor $d$ is a square in $\mathbf{Q}$. The elements of order 4 therefore double to $((1: 0),( \pm \sqrt{-1 / d}: 1))$.

If $s^{2}=-1 / d$, then the elements of order 4 doubling to $((1: 0),(s: 1))$ are $( \pm \sqrt{s}, \pm \sqrt{s})$ by Theorem 3.1 where the \pm signs are assumed independently. In particular, if such elements are defined over $\mathbf{Q}$, then $\pm \sqrt{s} \in \mathbf{Q}$, so $s$ is a square in $\mathbf{Q}$, so $-1 / d$ is a fourth power in $\mathbf{Q}$, say $f^{4}$. Now $( \pm f, \pm f)$ are points of order 4 doubling to $\left((1: 0),\left(f^{2}: 1\right)\right)$, and there are no other points of order 4 .

The torsion group contains a point $P_{8}$ of order 8 . This point doubles to $( \pm f, \pm f)$. Assume, without loss of generality, that $[2] P_{8}=( \pm f, f)$ : otherwise replace $f$ by $-f$. Further assume, without loss of generality, that $[2] P_{8}=(f, f)$; otherwise replace $P_{8}$ by $-P_{8}$. Any point having a zero coordinate has order at most 4 , so $P_{8}$ must be an affine point, say $\left(x_{8}, y_{8}\right)$, with $x_{8} \neq 0$ and $y_{8} \neq 0$.

Now $[2] P_{8}=(f, f)$ implies $\left(2 x_{8} y_{8}\right) /\left(-x_{8}^{2}+y_{8}^{2}\right)=f=\left(y_{8}^{2}+x_{8}^{2}\right) /\left(2+x_{8}^{2}-y_{8}^{2}\right)$, with $-x_{8}^{2}+y_{8}^{2} \neq 0$ and $2+x_{8}^{2}-y_{8}^{2} \neq 0$. In particular, $\left(y_{8}^{2}+x_{8}^{2}\right)\left(-x_{8}^{2}+y_{8}^{2}\right)=$ $\left(2 x_{8} y_{8}\right)\left(2+x_{8}^{2}-y_{8}^{2}\right)$, so $\left(y_{8}^{2}-x_{8}^{2}\right)\left(x_{8}^{2}+y_{8}^{2}+2 x_{8} y_{8}\right)=4 x_{8} y_{8}$; i.e., $\left(y_{8}^{2}-x_{8}^{2}\right) r^{2}=4 x_{8} y_{8}$ where $r=x_{8}+y_{8}$.

Define $s=2\left(y_{8}^{2}+x_{8}^{2}\right) /\left(y_{8}^{2}-x_{8}^{2}\right)$. Then

$$
s^{2}-4=\frac{4\left(\left(y_{8}^{2}+x_{8}^{2}\right)^{2}-\left(y_{8}^{2}-x_{8}^{2}\right)^{2}\right)}{\left(y_{8}^{2}-x_{8}^{2}\right)^{2}}=\frac{16 y_{8}^{2} x_{8}^{2}}{\left(y_{8}^{2}-x_{8}^{2}\right)^{2}}=r^{4}
$$

so $\left(s+r^{2}\right)^{2}-4=2 r^{2}\left(s+r^{2}\right)$; consequently, $\left(\left(s+r^{2}\right) / 2, r\left(s+r^{2}\right) / 2\right)$ is a rational point on the elliptic curve $\tau^{2}=\sigma^{3}-\sigma$. This curve has rank 0 over $\mathbf{Q}$ and exactly 3 
affine points over $\mathbf{Q}$, namely $( \pm 1,0)$ and $(0,0)$. Hence $r\left(s+r^{2}\right)=0$; consequently, $0=r\left(s+r^{2}\right)\left(s-r^{2}\right)=r\left(s^{2}-r^{4}\right)=4 r$, so $r=0$, so $x_{8}+y_{8}=0$, contradicting $-x_{8}^{2}+y_{8}^{2} \neq 0$.

Theorem 6.14. There exists no twisted Edwards curve over $\mathbf{Q}$ with torsion group isomorphic to $\mathbf{Z} / 10 \mathbf{Z}$.

Proof. Suppose that the twisted Edwards curve $\overline{\mathrm{E}}_{\mathrm{E}, a, d}: a x^{2}+y^{2}=1+d x^{2} y^{2}$ has $\mathbf{Q}$-torsion group isomorphic to $\mathbf{Z} / 10 \mathbf{Z}$. This means, in particular, that there exists a point $P_{5} \in \overline{\mathrm{E}}_{\mathrm{E}, a, d}(\mathbf{Q})$ of order 5 . Points at infinity have order at most 4 by Theorem 3.1 so $P_{5}=\left(x_{5}, y_{5}\right)$ for some $x_{5}, y_{5} \in \mathbf{Q}$. Points with a zero coordinate also have order at most 4 by Theorem 3.1. so $x_{5} \neq 0$ and $y_{5} \neq 0$. Note also that $y_{5} \notin\{-1,1\}$ since $x_{5} \neq 0$.

Apply the doubling formulas twice to see that the $x$-coordinate of [4] $P_{5}$ satisfies

$$
\begin{aligned}
& x\left([4] P_{5}\right)-\left(-x_{5}\right)= \\
& \frac{x_{5}\left(a x_{5}^{2}+y_{5}^{2}-2 y_{5}\right) F}{a^{4} x_{5}^{8}+4 y_{5}^{2} a^{3} x_{5}^{6}+\left(6 y_{5}^{4}-16 y_{5}^{2}\right) a^{2} x_{5}^{4}+\left(4 y_{5}^{6}-16 y_{5}^{4}+16 y_{5}^{2}\right) a x_{5}^{2}+y_{5}^{8}},
\end{aligned}
$$

where $F=a^{3} x_{5}^{6}+\left(3 y_{5}^{2}+6 y_{5}\right) a^{2} x_{5}^{4}+\left(3 y_{5}^{4}+4 y_{5}^{3}-4 y_{5}^{2}-8 y_{5}\right) a x_{5}^{2}+y_{5}^{6}-2 y_{5}^{5}-4 y_{5}^{4}$. The equation [4] $P_{5}=-P_{5}$ implies $x\left([4] P_{5}\right)-\left(-x_{5}\right)=0$, so $x_{5}\left(a x_{5}^{2}+y_{5}^{2}-2 y_{5}\right) F=0$.

Case 1: $a x_{5}^{2}+y_{5}^{2}=2 y_{5}$. Then $\left(x_{5},-y_{5}\right)$ is a curve point of order 3 by Theorem 3.3 , contradicting the hypothesis that the torsion group is isomorphic to $\mathbf{Z} / 10 \mathbf{Z}$.

Case 2: $F=0$. Define $q=\left(a x_{5}^{2}+y_{5}^{2}+2 y_{5}\right) / y_{5}$ and $r=q /\left(y_{5}+1\right)$. The identity $r q^{2}-\left(r^{2}+8\right) q+16=F /\left(y_{5}^{2}\left(y_{5}+1\right)^{2}\right)$ then implies $r q^{2}-\left(r^{2}+8\right) q+16=0$.

Define $U=q-r, V=q-r-4$, and $W=4-q-r$. Then $(U, V, W) \neq(0,0,0)$, and $V^{2} W-U^{3}-U^{2} W+U W^{2}=4\left(r q^{2}-\left(r^{2}+8\right) q+16\right)=0$, so $(U: V: W)$ is a rational point on the elliptic curve $\tau^{2}=\sigma^{3}+\sigma^{2}-\sigma$. This curve has rank 0 over $\mathbf{Q}$ and exactly 6 points over $\mathbf{Q}$, namely $( \pm 1, \pm 1),(0,0)$, and $(0: 1: 0)$, so $(U: V: W)$ is one of those points.

The points $(1,1)$ and $(-1,-1)$ and $(0,0)$ are excluded since $U \neq V$. The point $(1,-1)$ implies $(q, r)=(2,0)$, contradicting $r=q /\left(y_{5}+1\right)$. The point $(-1,1)$ implies $(q, r)=(4,2)$, again contradicting $r=q /\left(y_{5}+1\right)$ since $y_{5} \neq 1$. Finally, the point $(0: 1: 0)$ implies $(q, r)=(2,2)$, again contradicting $r=q /\left(y_{5}+1\right)$ since $y_{5} \neq 0$.

\section{EDWARDS CURVES WITH LARGE TORSION AND POSITIVE RANK}

Atkin and Morain in 3] found an infinite family of elliptic curves over $\mathbf{Q}$ with torsion group $\mathbf{Z} / 2 \mathbf{Z} \times \mathbf{Z} / 8 \mathbf{Z}$ and with explicit nontorsion points. Montgomery in [36. page 263] had earlier found an analogous family for $\mathbf{Z} / 12 \mathbf{Z}$. Suyama in 44] had earlier given an infinite sequence of Montgomery curves with explicit nontorsion points and with group order divisible by 12 over any prime field. GMP-ECM uses Suyama curves; see [47]. See [36, Section 6] for further $\mathbf{Z} / 2 \mathbf{Z} \times \mathbf{Z} / 8 \mathbf{Z}$ constructions.

Montgomery in [36, Section 6] selected various curves with torsion group $\mathbf{Z} / 12 \mathbf{Z}$, computed the group orders modulo primes $p$ in the interval $\left[10^{4}, 10^{5}\right]$, and found that the average exponents of 2 and 3 in the group orders were close to $11 / 3$ and $5 / 3$ respectively. We performed an analogous computation for primes in $\left[10^{6}, 2 \cdot 10^{6}\right]$, using Edwards curves with torsion group $\mathbf{Z} / 12 \mathbf{Z}$ constructed as in Section 6, and found an even closer match to $11 / 3$ and $5 / 3$. 
Most Suyama curves have $\mathbf{Q}$-torsion group only $\mathbf{Z} / 6 \mathbf{Z}$, and have averages only $10 / 3$ and 5/3. However, Kruppa discovered in 2007 that the Suyama curve with $\sigma=11$ has averages $11 / 3$ and $5 / 3$, despite having torsion group only $\mathbf{Z} / 6 \mathbf{Z}$; see 31 , Section 5.2.3].

In this section we translate the Atkin-Morain and Montgomery constructions from Weierstrass curves to Edwards curves. We also translate the Suyama construction to twisted Edwards curves.

This section relies on the equivalence in [8] between Montgomery curves and twisted Edwards curves. The twisted Edwards curve $\mathrm{E}_{\mathrm{E}, a, d}$ is birationally equivalent to the Montgomery curve $\mathrm{E}_{\mathrm{M}, A, B}: B v^{2}=u^{3}+A u^{2}+u$, where $A=$ $2(a+d) /(a-d)$ and $B=4 /(a-d)$. The map $(x, y) \mapsto(u, v)=((1+y) /(1-y)$, $(1+y) /((1-y) x))$ is a birational equivalence from $\mathrm{E}_{\mathrm{E}, a, d}$ to $\mathrm{E}_{\mathrm{M}, A, B}$, with inverse $(u, v) \mapsto(x, y)=(u / v,(u-1) /(u+1))$.

7.1. The Atkin-Morain construction. The Atkin-Morain family is parameterized by points $(s, t)$ on a particular elliptic curve $T^{2}=S^{3}-8 S-32$. Atkin and Morain suggest computing multiples $(s, t)$ of $(12,40)$, a nontorsion point on this curve. Beware that these points have rapidly increasing height.

Theorem 7.2 (Atkin, Morain). Let $(s, t)$ be a rational point on the curve $T^{2}=$ $S^{3}-8 S-32$. Define $\alpha=((t+25) /(s-9)+1)^{-1}, \beta=2 \alpha(4 \alpha+1) /\left(8 \alpha^{2}-1\right)$, $c=(2 \beta-1)(\beta-1) / \beta$, and $b=\beta c$. Then the elliptic curve

$$
E_{\alpha}: V^{2}=U^{3}+\frac{\left((c-1)^{2}-4 b\right)}{4} U^{2}+\frac{b(c-1)}{2} U+\frac{b^{2}}{4}
$$

has torsion group isomorphic to $\mathbf{Z} / 2 \mathbf{Z} \times \mathbf{Z} / 8 \mathbf{Z}$ and a point with $U$-coordinate $-(2 \beta-1) / 4$.

Theorem 7.3. Let $(s, t)$ be a rational point on the curve $T^{2}=S^{3}-8 S-32$. Define $\alpha$ and $\beta$ as in Theorem 7.2 . Define $d=\left(2(2 \beta-1)^{2}-1\right) /(2 \beta-1)^{4}$. Then the Edwards curve $x^{2}+y^{2}=1+d x^{2} y^{2}$ has torsion group isomorphic to $\mathbf{Z} / 2 \mathbf{Z} \times \mathbf{Z} / 8 \mathbf{Z}$ and a point $\left(x_{1}, y_{1}\right)$ with $x_{1}=(2 \beta-1)(4 \beta-3) /(6 \beta-5)$ and $y_{1}=(2 \beta-1)\left(t^{2}+\right.$ $\left.50 t-2 s^{3}+27 s^{2}-104\right) /((t+3 s-2)(t+s+16))$.

Proof. Put $x_{8}=2 \beta-1$. By construction $x_{8}$ satisfies $\left(2 x_{8}^{2}-1\right) / x_{8}^{4}=d$. Furthermore,

$$
d=\frac{\left(8 \alpha^{2}-1\right)^{2}\left(8 \alpha^{2}+8 \alpha+1\right)^{2}}{\left(8 \alpha^{2}+4 \alpha+1\right)^{4}},
$$

so $d$ is a square. By Theorem 6.6, the Edwards curve has torsion group isomorphic to $\mathbf{Z} / 2 \mathbf{Z} \times \mathbf{Z} / 8 \mathbf{Z}$. Finally, a straightforward calculation shows that $x_{1}^{2}+y_{1}^{2}=$ $1+d x_{1}^{2} y_{1}^{2}$.

The point with $U$-coordinate $-(2 \beta-1) / 4$ in Theorem 7.2 is generically a nontorsion point. The $V$-coordinate of the point is not stated explicitly in [3]. The point $\left(x_{1}, y_{1}\right)$ in Theorem 7.3 is the corresponding point on the Edwards curve.

7.4. The Suyama construction. The Suyama family has lower torsion but a simpler parameterization. We briefly review Suyama's family and present an analogous result for twisted Edwards curves.

Theorem 7.5 (Suyama). Let $\sigma>5$ be an integer. Define

$$
\alpha=\sigma^{2}-5, \quad \beta=4 \sigma, \quad W_{1}=\beta^{3}, \quad A=\frac{(\beta-\alpha)^{3}(3 \alpha+\beta)}{4 \alpha^{3} \beta}-2, \quad B=\frac{\alpha}{W_{1}} .
$$


Then the $\mathbf{Q}$-torsion group of the elliptic curve $\mathrm{E}_{\mathrm{M}, A, B}: B v^{2}=u^{3}+A u^{2}+u$ has a subgroup isomorphic to $\mathbf{Z} / 6 \mathbf{Z}$.

Define $V_{1}=\left(\sigma^{2}-1\right)\left(\sigma^{2}-25\right)\left(\sigma^{4}-25\right)$. Then $\left(u_{1}, v_{1}\right)=\left(\alpha^{3} / W_{1}, V_{1} / W_{1}\right)$ is a nontorsion point on $\mathrm{E}_{\mathrm{M}, A, B}$.

Theorem 7.6. Let $\sigma>5$ be an integer. Define $\alpha, \beta, V_{1}$ as in Theorem 7.5, Define $a=(\beta-\alpha)^{3}(3 \alpha+\beta)$ and $d=(\beta+\alpha)^{3}(\beta-3 \alpha)$. Then the $\mathbf{Q}$-torsion group of the twisted Edwards curve ax $x^{2}+y^{2}=1+d x^{2} y^{2}$ has a subgroup isomorphic to $\mathbf{Z} / 6 \mathbf{Z}$, and $\left(x_{1}, y_{1}\right)=\left(\alpha \beta /\left(2 V_{1}\right),\left(\alpha^{3}-\beta^{3}\right) /\left(\alpha^{3}+\beta^{3}\right)\right)$ is a nontorsion point on the curve.

Proof. Define $W_{1}, A, B$ as in Theorem 7.5. Then $2(a+d) /(a-d)=A$ and $4 /(a-d)=$ $B \beta^{2} /\left(2 \alpha^{2}\right)^{2}$. The twisted Edwards curve $a x^{2}+y^{2}=1+d x^{2} y^{2}$ is birationally equivalent to the Montgomery curve $\left(B \beta^{2} /\left(2 \alpha^{2}\right)^{2}\right) v^{2}=u^{3}+A u^{2}+u$, which in turn is isomorphic to the Montgomery curve $B v^{2}=u^{3}+A u^{2}+u$, so its $\mathbf{Q}$-torsion group has a subgroup isomorphic to $\mathbf{Z} / 6 \mathbf{Z}$ by Theorem 7.5

Define $u_{1}, v_{1}$ as in Theorem 7.5. Then $\left(u_{1}, v_{1}\right)$ is a nontorsion point on $B v^{2}=$ $u^{3}+A u^{2}+u$, so $\left(u_{1}, v_{1}\left(2 \alpha^{2}\right) / \beta\right)$ is a nontorsion point on $\left(B \beta^{2} /\left(2 \alpha^{2}\right)^{2}\right) v^{2}=u^{3}+$ $A u^{2}+u$. Mapping this point to $\mathrm{E}_{\mathrm{E}, a, d}$ yields exactly $\left(x_{1}, y_{1}\right)$ :

$$
x_{1}=\frac{u_{1}}{v_{1}\left(2 \alpha^{2}\right) / \beta}=\frac{\alpha^{3}}{V_{1}\left(2 \alpha^{2}\right) / \beta}=\frac{\alpha \beta}{2 V_{1}} \quad \text { and } \quad y_{1}=\frac{u_{1}-1}{u_{1}+1}=\frac{\alpha^{3}-\beta^{3}}{\alpha^{3}+\beta^{3}} \text {. }
$$

Hence $\left(x_{1}, y_{1}\right)$ is a nontorsion point on $a x^{2}+y^{2}=1+d x^{2} y^{2}$.

7.7. The Montgomery construction. Montgomery's family is parameterized by points $(s, t)$ on the curve $T^{2}=S^{3}-12 S$, except for the points $(0,0), Q=(-2,-4)$, $R=(6,-12),-Q$, and $-R$. Observe that this curve has $\operatorname{rank} 1$, that $Q$ is a nontorsion point on the curve, and that $R=Q+(0,0)$ is not in the cyclic group generated by $Q$, so one way to generate infinitely many suitable points on the curve is to compute $[2] Q,[3] Q$, etc. Montgomery does not state any particular generation mechanism but gives five examples in [36, page 263] that appear to have been generated as $[2] R,[3] R,[4] R,[5] R$, and $[6] R$.

Theorem 7.8. Let $(s, t) \notin\{(0,0),(-2, \pm 4),(6, \pm 12)\}$ be a rational point on the curve $T^{2}=S^{3}-12 S$. Define

$$
d=\frac{-(s-2)^{3}(s+6)^{3}\left(s^{2}-12 s-12\right)}{1024 s^{2} t^{2}} .
$$

Then the Edwards curve $E: x^{2}+y^{2}=1+d x^{2} y^{2}$ has $\mathbf{Q}$-torsion group isomorphic to $\mathbf{Z} / 12 \mathbf{Z}$ and has a nontorsion point $\left(x_{1}, y_{1}\right)$ where

$$
x_{1}=\frac{8 t\left(s^{2}+12\right)}{(s-2)(s+6)\left(s^{2}+12 s-12\right)} \quad \text { and } \quad y_{1}=\frac{-4 s\left(s^{2}-12 s-12\right)}{(s-2)(s+6)\left(s^{2}-12\right)} .
$$

Proof. Note that the restrictions on $(s, t)$ ensure that all denominators are nonzero and that $d \notin\{0,1\}$.

Define $x_{3}=4 t /((s-2)(s+6))$ and $y_{3}=-8 s /((s-2)(s+6))$. A straightforward calculation shows that $x_{3}^{2}+y_{3}^{2}=1+d x_{3}^{2} y_{3}^{2}=-2 y_{3}$, so $\left(x_{3}, y_{3}\right)$ is a point of order 3 on $E$ by Theorem 3.3 , so the torsion group of $E$ has a subgroup isomorphic to $\mathbf{Z} / 12 \mathbf{Z}$. By Mazur's theorem, the torsion group of $E$ is isomorphic to $\mathbf{Z} / 12 \mathbf{Z}$. 
Another straightforward calculation shows that $x_{1}^{2}+y_{1}^{2}=1+d x_{1}^{2} y_{1}^{2}$, so $\left(x_{1}, y_{1}\right)$ is a curve point. If it were a torsion point, then the ratio $\left(x_{1}: y_{1}\right)=\left(-2 t\left(s^{2}-\right.\right.$ $\left.12)\left(s^{2}+12\right): s\left(s^{2}-12 s-12\right)\left(s^{2}+12 s-12\right)\right)$ would have to be equal to $(0: 1)$ or $(1: 0)$ or $\left( \pm x_{3}: y_{3}\right)=( \pm t: 2 s)$ or $\left( \pm y_{3}: x_{3}\right)=\left( \pm 2 t: s^{2}-12\right)$ by Theorem 6.3 this is also avoided by the restrictions on $(s, t)$.

\section{EdWARds CURVES With SMALl PARAMETERs, LARGE TORSION, AND POSITIVE RANK}

One way to save time in computations on twisted Edwards curves is to choose small curve parameters $a$ and $d$ and a small-height nontorsion base point $\left(X_{1}: Y_{1}\right.$ : $Z_{1}$ ); see Section 2.8. Another way to save time is to construct curves with large Q-torsion group and positive rank; see Section 7. Unfortunately, essentially all of the $\mathbf{Z} / 12 \mathbf{Z}$ and $\mathbf{Z} / 2 \mathbf{Z} \times \mathbf{Z} / 8 \mathbf{Z}$ curves constructed in Section 7 have $a, d, X_{1}, Y_{1}, Z_{1}$ of large height.

Our aim in this section is to combine these two time-saving techniques, finding twisted Edwards curves that simultaneously have small parameters $a, d$, a smallheight nontorsion point $\left(X_{1}: Y_{1}: Z_{1}\right)$, and large torsion over $\mathbf{Q}$.

Overall we found more than 100 small Edwards curves having small-height nontorsion points and at least 12 torsion points over $\mathbf{Q}$. For the complete list see http://eecm.cr.yp.to/goodcurves.html. The number of $d$ 's below height $H$ appears to grow as roughly $\log H$; for comparison, the Atkin-Morain procedure discussed in Section 7 generates only about $\sqrt{\log H}$ examples below height $H$. Of course, one can easily write down many more small curves if one is willing to sacrifice some torsion, as illustrated by the Suyama construction.

8.1. Torsion group $\mathbf{Z} / 12 \mathbf{Z}$. Theorem 6.4 gives a complete parameterization of all Edwards curves with torsion group isomorphic to $\mathbf{Z} / 12 \mathbf{Z}$. Any rational point $\left(u, x_{3}, y_{3}, d, x_{1}, y_{1}\right)$ on the surface described by

$$
x_{3}=\frac{u^{2}-1}{u^{2}+1}, y_{3}=-\frac{(u-1)^{2}}{u^{2}+1}, d=\frac{\left(u^{2}+1\right)^{3}\left(u^{2}-4 u+1\right)}{(u-1)^{6}(u+1)^{2}}, x_{1}^{2}+y_{1}^{2}=1+d x_{1}^{2} y_{1}^{2}
$$

gives us a suitable curve for ECM if $u \notin\{0, \pm 1\}$ and $\left(x_{1}, y_{1}\right)$ is not a torsion point. Theorem 6.3 lists all affine torsion points.

Assume, without loss of generality, that $|u|>1$ : otherwise replace $u$ by $1 / u$, obtaining the same $d$. Write $u$ as $a / b$ for integers $a, b$ satisfying $0<|b|<a$. Define $e=\left(a^{2}-b^{2}\right) / x_{1}$ and $f=-(a-b)^{2} / y_{1}$, and assume, without loss of generality, that $e, f$ are integers: otherwise scale $a, b$ appropriately. The curve equation $x_{1}^{2}+y_{1}^{2}=1+$ $d x_{1}^{2} y_{1}^{2}$ now implies, after some simplification, the $(1,1,2,2)$-weighted-homogeneous equation

$$
\left(e^{2}-\left(a^{2}-b^{2}\right)^{2}\right)\left(f^{2}-(a-b)^{4}\right)=16 a^{3} b^{3}\left(a^{2}-a b+b^{2}\right) .
$$

We found many small solutions to this equation, and thus many of the desired Edwards curves, as follows. We considered a range of positive integers $a$. For each $a$ we enumerated integers $b$ with $0<|b|<a$. For each $(a, b)$ we enumerated all divisors of $16 a^{3} b^{3}\left(a^{2}-a b+b^{2}\right)$ and added $\left(a^{2}-b^{2}\right)^{2}$ to each divisor. For each sum of the form $e^{2}$ we added $(a-b)^{4}$ to the complementary divisor, checked for a square, checked that the corresponding $\left(x_{1}, y_{1}\right)$ was a nontorsion point, etc. 
After about a week of computation on some computers at LORIA we had found 78 different values of $d$ and checked that we had 78 different $j$-invariants. Here are two examples:

- the very small solution $(a, b, e, f)=(3,2,23,7)$ produces the order-3 point $\left(x_{3}, y_{3}\right)=(5 / 13,-1 / 13)$ and the nontorsion point $\left(x_{1}, y_{1}\right)=(5 / 23,-1 / 7)$ on the Edwards curve $x^{2}+y^{2}=1+d x^{2} y^{2}$ where $d=-11 \cdot 13^{3} / 5^{2}$;

- the solution $(a, b, e, f)=(15180,-7540,265039550,161866240)$ produces the nontorsion point $(3471616 / 5300791,-201640 / 63229)$ on the Edwards curve $x^{2}+y^{2}=1+d x^{2} y^{2}$ where $d=931391 \cdot 359105^{3} / 140003330048^{2}$.

8.2. Torsion group $\mathbf{Z} / 2 \mathbf{Z} \times \mathbf{Z} / 8 \mathbf{Z}$. Theorem 6.9 gives a complete parameterization of all Edwards curves with torsion group isomorphic to $\mathbf{Z} / 2 \mathbf{Z} \times \mathbf{Z} / 8 \mathbf{Z}$ and with a point of order 8 doubling to $( \pm 1,0)$. Any rational point $\left(u, x_{8}, d, x_{1}, y_{1}\right)$ on the surface described by $x_{8}=\left(u^{2}+2 u+2\right) /\left(u^{2}-2\right), d=\left(2 x_{8}^{2}-1\right) / x_{8}^{4}$, and $x_{1}^{2}+y_{1}^{2}=$ $1+d x_{1}^{2} y_{1}^{2}$ gives us a suitable curve for ECM if $u \notin\{0,-1,-2\}$ and $\left(x_{1}, y_{1}\right)$ is not a torsion point. Theorem 6.8 lists all affine torsion points.

We consider only $u>\sqrt{2}$. Various transformations of $u$ listed in Theorem 6.9 show that this does not lose any generality: if $0<u<\sqrt{2}$, then $2 / u>\sqrt{2}$, and $2 / u$ produces the same curve; if $u<-2$, then $-(u+2)>0$, and $-(u+2)$ produces the same curve; if $-2<u<-1$, then $-2(u+1) /(u+2)>0$, and $-2(u+1) /(u+2)$ produces the same curve; if $-1<u<0$, then $-u /(u+1)>0$, and $-u /(u+1)$ produces the same curve.

Write $u=a / b, x_{1}=\left(a^{2}+2 a b+2 b^{2}\right) / e$, and $y_{1}=\left(a^{2}+2 a b+2 b^{2}\right) / f$ where $a, b, e, f$ are integers. Then $a, b, e, f$ satisfy the $(1,1,2,2)$-weighted-homogeneous equation

$$
\left(e^{2}-\left(a^{2}+2 a b+2 b^{2}\right)^{2}\right)\left(f^{2}-\left(a^{2}+2 a b+2 b^{2}\right)^{2}\right)=(4 a b(a+b)(a+2 b))^{2} .
$$

We found many small solutions to this equation, and thus many of the desired Edwards curves, by a procedure similar to the procedure used for $\mathbf{Z} / 12 \mathbf{Z}$. We considered a range of positive integers $a$. For each $a$ we enumerated integers $b$ between 1 and $\lfloor a / \sqrt{2}\rfloor$. For each $(a, b)$ we enumerated all divisors of $(4 a b(a+$ b) $(a+2 b))^{2}$, added $\left(a^{2}+2 a b+2 b^{2}\right)^{2}$ to each divisor, and searched for squares.

After about a week of computation on some computers at LORIA, we had found 25 different values of $d$ and checked that we had 25 different $j$-invariants. Here are two examples:

- the very small solution $(a, b, e, f)=(3,1,19,33)$ produces the order-8 point $\left(x_{8}, x_{8}\right)=(17 / 7,17 / 7)$ and the nontorsion point $\left(x_{1}, y_{1}\right)=(17 / 19,17 / 33)$ on the Edwards curve $x^{2}+y^{2}=1+d x^{2} y^{2}$ where $d=161^{2} / 17^{4}$;

- the solution $(a, b, e, f)=(24882,9009,258492663,580153002)$ produces the nontorsion point $\left(x_{1}, y_{1}\right)=(86866 / 18259,8481 / 4001)$ on the Edwards curve $x^{2}+y^{2}=1+d x^{2} y^{2}$ where $d=5657719^{2} / 3341^{4}$.

\section{THE IMPACT OF LARGE TORSION}

This section reports various measurements of the success probability of EECMMPFQ. These measurements demonstrate the importance of choosing a curve with a large torsion group. They also demonstrate the inaccuracy of several common methods of estimating the success probability of ECM. 
9.1. Impact of torsion for 20-bit primes. There are exactly 38635 primes between $2^{19}$ and $2^{20}$. As an experiment we fed each of these primes to EECM-MPFQ with $B_{1}=256$ and $d_{1}=1$. It turned out that the first curve configured into EECM-MPFQ finds 12467, i.e., $32.2687 \%$, of these primes. This curve is the Edwards curve $x^{2}+y^{2}=1-(24167 / 25) x^{2} y^{2}$, with base point $P=(5 / 23,-1 / 7)$; this curve has torsion group isomorphic to $\mathbf{Z} / 12 \mathbf{Z}$.

We then modified EECM-MPFQ to instead start with the curve $x^{2}+y^{2}=$ $1+(25921 / 83521) x^{2} y^{2}$, with base point $P=(13 / 7,289 / 49)$, and repeated the same experiment. This curve has torsion group isomorphic to $\mathbf{Z} / 2 \mathbf{Z} \times \mathbf{Z} / 8 \mathbf{Z}$; it is one of the curves that EECM-MPFQ normally tries, although not the first in the list. This curve finds $32.8433 \%$ of the primes.

We then made a more drastic modification to EECM-MPFQ, trying two new curves with smaller torsion groups. The curve $x^{2}+y^{2}=1+(1 / 36) x^{2} y^{2}$, with base point $P=(8,9)$, has torsion group only $\mathbf{Z} / 2 \mathbf{Z} \times \mathbf{Z} / 4 \mathbf{Z}$ and finds only $27.4854 \%$ of the primes, losing a factor 1.17 compared to the original $\mathbf{Z} / 12 \mathbf{Z}$ curve. The curve $x^{2}+y^{2}=1+(1 / 3) x^{2} y^{2}$, with base point $P=(2,3)$, has torsion group only $\mathbf{Z} / 4 \mathbf{Z}$ and finds only $23.4709 \%$ of the primes, losing a factor 1.37 compared to the original $\mathbf{Z} / 12 \mathbf{Z}$ curve.

9.2. Impact of torsion for 30-bit primes. As a larger experiment we replaced the 3863520 -bit primes by a random sample of 65536 distinct 30-bit primes and increased $\left(B_{1}, d_{1}\right)$ from $(256,1)$ to $(1024,1)$. The same four curves again had remarkably different performance:

- $12.1597 \%$ of the primes were found by the $\mathbf{Z} / 12 \mathbf{Z}$ curve.

- $11.9751 \%$ of the primes were found by the $\mathbf{Z} / 2 \mathbf{Z} \times \mathbf{Z} / 8 \mathbf{Z}$ curve.

- $9.8465 \%$ of the primes were found by the $\mathbf{Z} / 2 \mathbf{Z} \times \mathbf{Z} / 4 \mathbf{Z}$ curve.

- $9.0073 \%$ of the primes were found by the $\mathbf{Z} / 4 \mathbf{Z}$ curve.

For comparison, GMP-ECM with a typical Suyama curve (specifically $\sigma=10$ ) finds $11.6837 \%$ of the same primes. We also tried GMP-ECM's Pollard $p-1$ option; it found $6.3507 \%$ of the same primes. Normally the $p-1$ method is assumed to be a helpful first step before ECM, because it uses fewer multiplications per bit than an elliptic curve, but we comment that this benefit is reduced by the $p-1$ curve (a hyperbola) having torsion group only $\mathbf{Z} / 2 \mathbf{Z}$.

Figures 9.1 and 9.2 show the results of similar measurements for the same four EECM curves for many prime powers $B_{1}$ : specifically, every prime power $B_{1} \leq 500$ for the 20 -bit primes, and every prime power $B_{1} \leq 2000$ for the 30 -bit primes. The figures show that $\mathbf{Z} / 12 \mathbf{Z}$ (black) and $\mathbf{Z} / 2 \mathbf{Z} \times \mathbf{Z} / 8 \mathbf{Z}$ (blue) are consistently better than $\mathbf{Z} / 2 \mathbf{Z} \times \mathbf{Z} / 4 \mathbf{Z}$ (blue, lower) and $\mathbf{Z} / 4 \mathbf{Z}$ (black, lower).

The figures also include measurements for the same GMP-ECM Suyama curve (red) and $p-1$ (red, lower). When $B_{1}$ is large, the EECM-MPFQ Z/12Z and $\mathbf{Z} / 2 \mathbf{Z} \times \mathbf{Z} / 8 \mathbf{Z}$ curves find significantly more primes than the GMP-ECM Suyama curve.

9.3. Review of methods of estimating the success probability. Consider the fraction of primes $p \in[L, R]$ found by stage 1 of ECM with a particular curve $E$, point $P \in E(\mathbf{Q})$, and smoothness bound $B_{1}$. Assume that $E$ is chosen to guarantee $t$ as a divisor of $\# E\left(\mathbf{F}_{p}\right)$. 
Standard practice in the literature is to estimate this fraction through the following series of heuristic approximations, where $\langle P \bmod p\rangle$ means the subgroup of $E\left(\mathbf{F}_{p}\right)$ generated by $P$ :

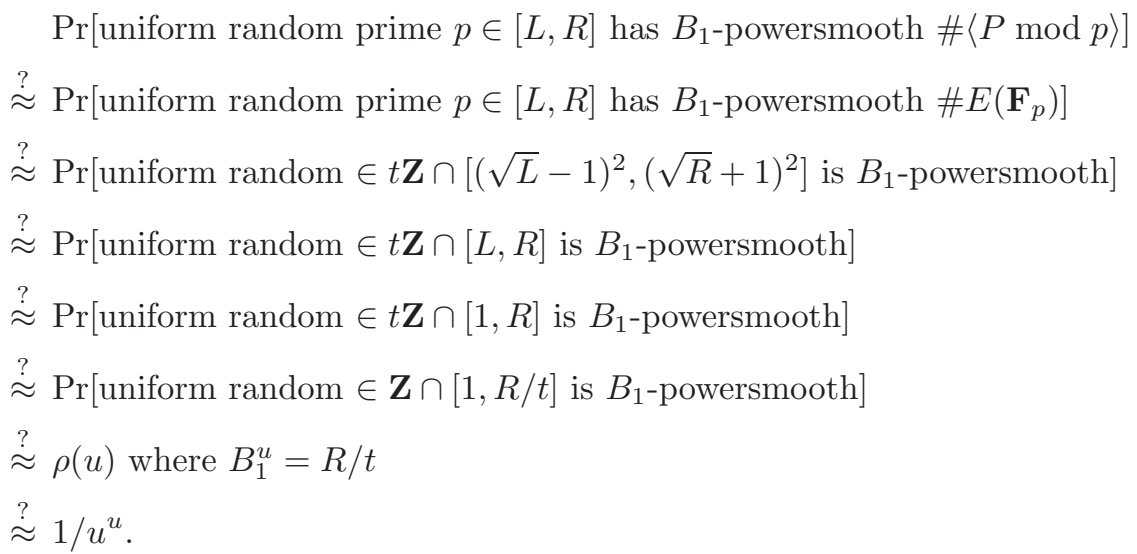

Here " $B_{1}$-powersmooth" means "having no prime-power divisors larger than $B_{1}$," and $\rho$ is Dickman's rho function introduced in 24. Similar comments apply to stage 2 , with $B_{1}$-powersmoothness replaced by a more complicated notion of smoothness and with $\rho$ replaced by a standard generalization.

For example, Montgomery in [37, Section 7] estimated the success chance of a curve with 16 torsion points over $\mathbf{Q}$ as the $B_{1}$-powersmoothness chance for a uniform random integer in $[1, p / 16]$. Similarly, Silverman and Wagstaff in [41] estimated the success chance of a Suyama curve as the $B_{1}$-powersmoothness chance for a uniform random integer in $[1, p / 12]$, following Brent's comment in [18, Section 9.3] that choosing a Suyama curve "effectively reduces $p$ to $p / 12$ in the analysis." (As mentioned in Section 7 a typical Suyama curve has only 6 torsion points over $\mathbf{Q}$, but a Suyama curve modulo $p$ is guaranteed to have order in 12Z.) Brent, Montgomery, et al. used Dickman's rho function to estimate the $B_{1}$-powersmoothness chance for a uniform random integer.

9.4. Inaccuracy of the estimates. There are many reasons to question the accuracy of the above approximations:

- Dickman's rho function $\rho$ is asymptotically $1 / u^{u}$ in the loose sense that $(\log \rho(u)) /(-u \log u) \rightarrow 1$ as $u \rightarrow \infty$, but is not actually very close to $1 / u^{u}$ : for example, $\rho(2) \approx 1.23 / 2^{2}, \rho(3) \approx 1.31 / 3^{3}$, and $\rho(4) \approx 1.26 / 4^{4}$.

- For each $u \geq 0$, the $B_{1}$-smoothness probability for an integer in $\left[1, B_{1}^{u}\right]$ converges to $\rho(u)$ as $B_{1} \rightarrow \infty$, and the same is true for $B_{1}$-powersmoothness, but the convergence is actually quite slow.

- Multiplying an element of $\mathbf{Z} \cap[1, R / 16]$ by 16 never gains powersmoothness but can lose powersmoothness when the original exponent of 2 was large, not an uncommon event among powersmooth integers.

- The ratio of smoothness probabilities for (e.g.) $\left[1, B_{1}^{u}\right]$ and $\left[(1 / 2) B_{1}^{u}, B_{1}^{u}\right]$ converges to 1 as $B_{1} \rightarrow \infty$, but the convergence is again quite slow. 
- Lenstra commented in [33, page 660] that an elliptic curve has even order with probability approximately $2 / 3$, not $1 / 2$. Many subsequent reports (for example, by Brent in [18, Table 3] and McKee in [35, Section 2]) have lent support to the idea that elliptic-curve orders are somewhat more likely to be smooth than uniform random integers.

- The group order $\# E\left(\mathbf{F}_{p}\right)$ is a multiple of the point order $\#\left\langle P\right.$ in $\left.E\left(\mathbf{F}_{p}\right)\right\rangle$. The ratio is usually small but often enough to change powersmoothness, as illustrated by the $s=420$ example in Section 4.1

The overall error is not extremely large but can easily be large enough to interfere with optimization.

Recall that the curve $x^{2}+y^{2}=1-(24167 / 25) x^{2} y^{2}$, with 12 torsion points, finds $32.2687 \%$ of the primes in $\left[2^{19}, 2^{20}\right]$ with $B_{1}=256$ and $d_{1}=1$; and that changing to three other curves with 16,8 , and 4 torsion points changes $32.2687 \%$ to $32.8433 \%, 27.4854 \%$, and $23.4709 \%$ respectively. We computed several of the standard estimates for these four success probabilities:

- A uniform random element of $12 \mathbf{Z} \cap\left[2^{19}, 2^{20}\right]$ has a $23.6067 \%$ chance of being 256-powersmooth. Note that this probability drastically underestimates the actual ECM smoothness chance. Changing 12 to 16, 8, 4 changes $23.6067 \%$ to $24.8192 \%, 20.5777 \%$, and $16.8006 \%$ respectively.

- A uniform random element of $12 \mathbf{Z} \cap\left[1,2^{20}\right]$ has a $30.0317 \%$ chance of being 256-powersmooth. Changing 12 to $16,8,4$ changes $30.0317 \%$ to $31.3019 \%$, $26.4328 \%$, and $21.8632 \%$ respectively.

- A uniform random element of $\mathbf{Z} \cap\left[1,2^{20} / 12\right]$ has a $30.7652 \%$ chance of being 256-powersmooth. Changing 12 to $16,8,4$ changes $30.7652 \%$ to $33.3694 \%$, $27.3689 \%$, and $22.2511 \%$ respectively.

- If $u=\left(\log \left(2^{20} / 12\right)\right) / \log 256$, then $\rho(u) \approx 28.1894 \%$. Changing 12 to $16,8,4$ changes $28.1894 \%$ to $30.6853 \%, 24.9832 \%$, and $20.2442 \%$ respectively.

- If $u=\left(\log \left(2^{20} / 12\right)\right) / \log 256$, then $u^{-u} \approx 22.8824 \%$. Changing 12 to $16,8,4$ changes $22.8824 \%$ to $25 \%, 20.1540 \%$, and $16.1283 \%$ respectively.

These approximations make 16 seem better than 12 by factors of 1.051, 1.042, 1.085, 1.089, and 1.093, when in fact 16 is better than 12 by a factor of only 1.018.

Figure 9.1 includes, for many prime powers $B_{1}$, the $B_{1}$-powersmoothness chance of a uniform random element of $t \mathbf{Z} \cap\left[2^{19}, 2^{20}\right]$ for four values of $t$ (green and gray graphs, bumpy), and $\rho\left(\left(\log \left(2^{20} / t\right)\right) / \log B_{1}\right)$ for four values of $t$ (green and gray graphs, smooth). Figure 9.2 includes analogous results for 30 -bit primes. It is clear that the $\rho$ value is a poor approximation to the powersmoothness chance, and that the powersmoothness chance is a poor approximation to the ECM success chance.

One can ask whether better approximations are possible. We comment that a fast algorithm to compute tight bounds on smoothness probabilities appeared in [5], and that the same algorithm can be adapted to handle powersmoothness, local conditions such as more frequent divisibility by 2, etc. However, one can also ask whether approximations are necessary in the first place. ECM is most frequently used to find rather small primes (for example, inside the number-field sieve), and for those primes one can simply measure ECM's performance by experiment. 


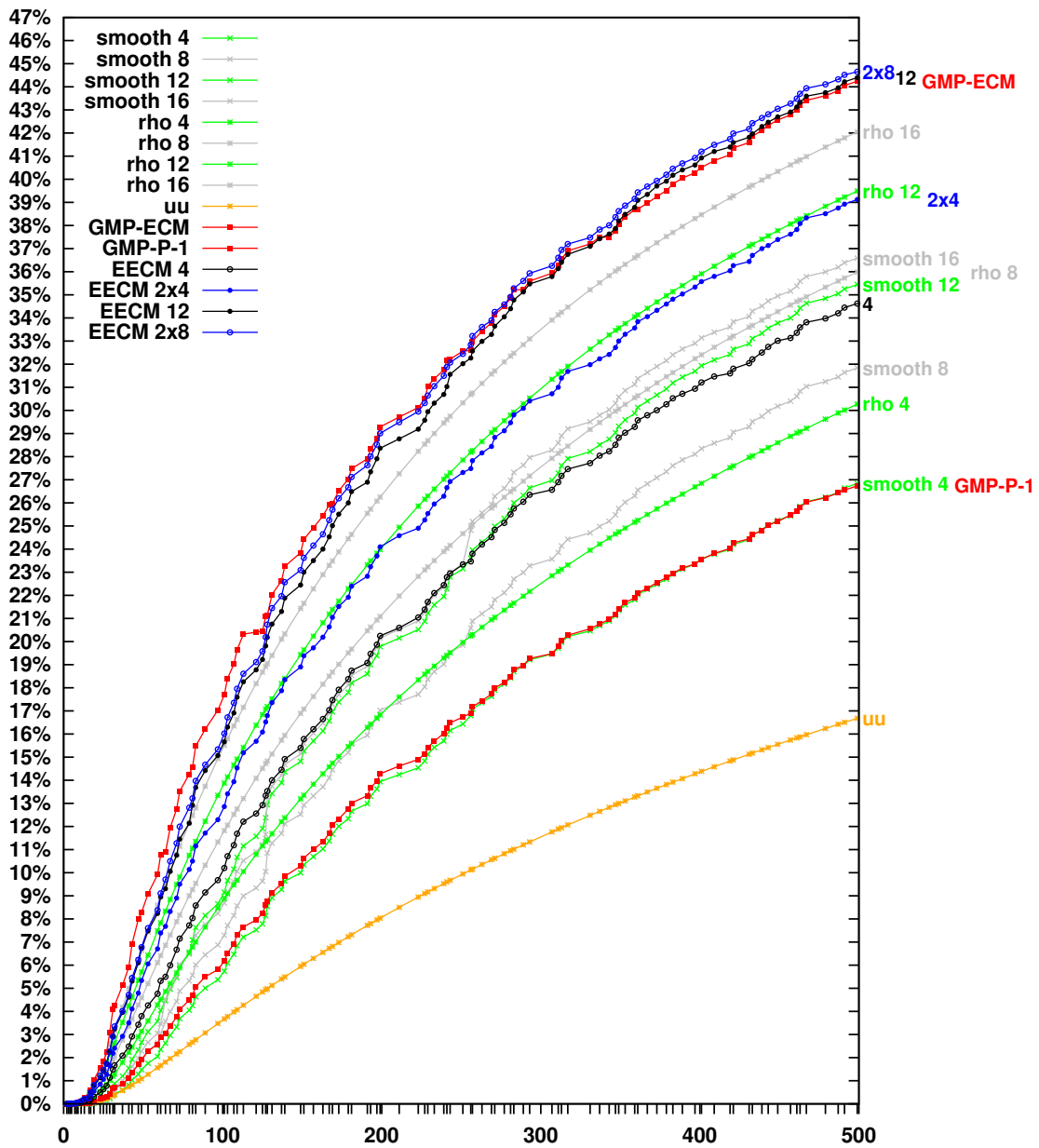

Figure 9.1. For the set of all 38635 20-bit primes: Measured stage-1 success probabilities for six curves, and nine estimates. Horizontal axis is $B_{1}$. Vertical axis is probability. Graphs from top to bottom on right side: (blue, bumpy) EECM-MPFQ with a $\mathbf{Z} / 2 \mathbf{Z} \times \mathbf{Z} / 8 \mathbf{Z}$ curve; (black, bumpy) EECM-MPFQ with a $\mathbf{Z} / 12 \mathbf{Z}$ curve; (red, bumpy) GMP-ECM with a Suyama curve; (gray, smooth) the $\rho$ approximation to smoothness probability for $\left[1,2^{20} / 16\right]$; (green, smooth) the $\rho$ approximation for $\left[1,2^{20} / 12\right]$; (blue, bumpy) EECM-MPFQ with a $\mathbf{Z} / 2 \mathbf{Z} \times \mathbf{Z} / 4 \mathbf{Z}$ curve; (gray, bumpy) powersmoothness probability for $16 \mathbf{Z} \cap\left[2^{19}, 2^{20}\right]$; (gray, smooth) the $\rho$ approximation for $\left[1,2^{20} / 8\right]$; (green, bumpy) powersmoothness probability for $12 \mathbf{Z} \cap\left[2^{19}, 2^{20}\right]$; (black, bumpy) EECM-MPFQ with a $\mathbf{Z} / 4 \mathbf{Z}$ curve; (gray, bumpy) powersmoothness probability for $8 \mathbf{Z} \cap\left[2^{19}, 2^{20}\right]$; (green, smooth) the $\rho$ approximation for $\left[1,2^{20} / 4\right]$; (green, bumpy) powersmoothness probability for $4 \mathbf{Z} \cap\left[2^{19}, 2^{20}\right]$; (red, bumpy) GMPECM with $p-1$; (orange, smooth) the $u^{-u}$ approximation for $\left[1,2^{20}\right]$. 


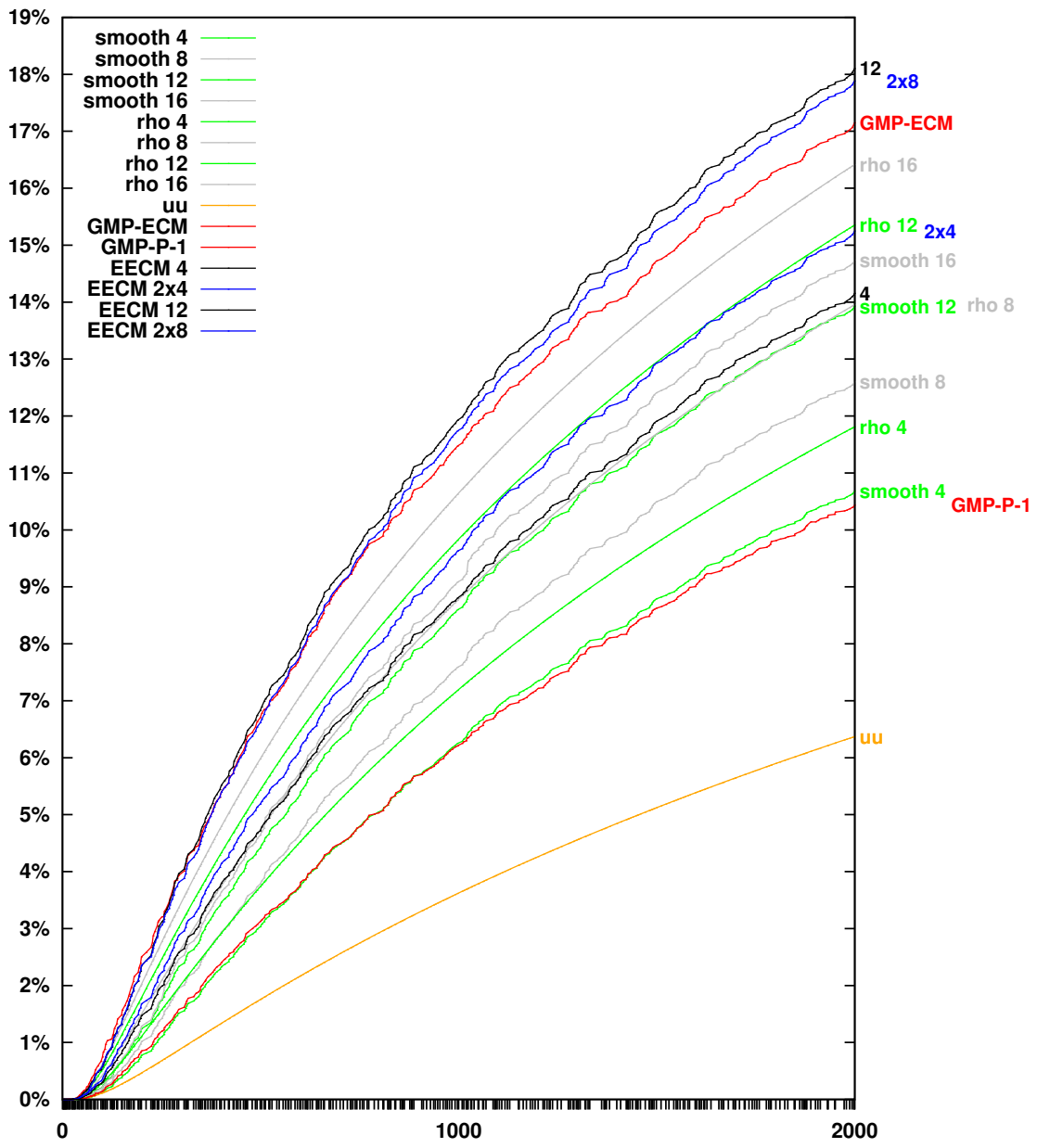

Figure 9.2. For a sample of 65536 30-bit primes: Measured stage-1 success probabilities for six curves, and nine estimates. Horizontal axis is $B_{1}$. Vertical axis is probability. Graphs from top to bottom on right side: (black, bumpy) EECMMPFQ with a $\mathbf{Z} / 2 \mathbf{Z} \times \mathbf{Z} / 8 \mathbf{Z}$ curve; (blue, bumpy) EECM-MPFQ with a $\mathbf{Z} / 12 \mathbf{Z}$ curve; (red, bumpy) GMP-ECM with a Suyama curve; (gray, smooth) the $\rho$ approximation to smoothness probability for $\left[1,2^{30} / 16\right]$; (green, smooth) the $\rho$ approximation for [1, $\left.2^{30} / 12\right]$; (blue, bumpy) EECM-MPFQ with a $\mathbf{Z} / 2 \mathbf{Z} \times \mathbf{Z} / 4 \mathbf{Z}$ curve; (gray, bumpy) powersmoothness probability for $16 \mathbf{Z} \cap\left[2^{29}, 2^{30}\right]$; (black, bumpy) EECM-MPFQ with a Z/4Z curve; (gray, smooth) the $\rho$ approximation for $\left[1,2^{30} / 8\right]$; (green, bumpy) powersmoothness probability for $12 \mathbf{Z} \cap\left[2^{29}, 2^{30}\right]$; (gray, bumpy) powersmoothness probability for $8 \mathbf{Z} \cap\left[2^{29}, 2^{30}\right]$; (green, smooth) the $\rho$ approximation for $\left[1,2^{30} / 4\right]$; (green, bumpy) powersmoothness probability for $4 \mathbf{Z} \cap\left[2^{29}, 2^{30}\right]$; (red, bumpy) GMP-ECM with $p-1$; (orange, smooth) the $u^{-u}$ approximation for $\left[1,2^{30}\right]$. 


\section{Choosing Parameters}

This section reports EECM-MPFQ's overall performance at finding various sizes of primes, when the parameters $B_{1}, d_{1}$, etc. are chosen sensibly.

10.1. Normalizing the success probability. Stage 1 will almost never find any factors of $n$ if $B_{1}$ is very small, and stage 2 will almost never find any factors of $n$ if $d_{1}$ and $e$ are very small. The success probability increases as the parameters increase, and eventually reaches 1 (for any particular size of prime); however, the costs of stage 1 and stage 2 then become enormously large. It is generally best to use intermediate parameters that balance the cost of each curve against the success probability of the curve, and to compensate for a low success probability by trying several curves.

Montgomery in [36, Table 7.4.1] computed an "expected time" obtained by multiplying an "expected number of curves" by an "estimated time per curve". The "expected number of curves" was the reciprocal of an estimate of the success probability per curve. The "estimated time per curve" was $5.5 B_{1}$ milliseconds for stage 1, $105 d_{1}$ milliseconds for initial elliptic-curve operations in stage 2, etc. Montgomery selected the constants 5.5, 105, etc. to approximately fit timings of his ECM implementation on a DEC 5000.

We instead report actual measurements of EECM-MPFQ's price-performance ratio. Specifically, we report the actual number of modular multiplications used by an EECM-MPFQ curve for both stage 1 and stage 2, divided by the actual success probability of that curve within a target set of primes. To simplify these reports we count $\mathbf{S}$ as $\mathbf{M}$, we count multiplications by small numbers (such as the coordinates of the base point) as $\mathbf{M}$, and we skip the fast-polynomial-arithmetic variant described in Section 5.3 .

Our experiments actually used slightly fewer modular multiplications per prime, because primes found in stage 1 did not incur the costs of stage 2 (and primes found during the batched division in stage 2 did not incur the remaining costs of stage 2). This cost reduction is reported as "savings" in Table 10.1 below. An application that uses EECM with a similar distribution of primes within its inputs will see a similar savings. On the other hand, an application faced with a large pool of inputs, where primes of the desired size appear within relatively few inputs, will see smaller savings.

We also report, later in the section, the number of clock cycles used by EECMMPFQ for both stage 1 and stage 2, again divided by success probability. The number of multiplications per prime found is a simpler measure than the number of cycles per prime found, and is an adequate measure for seeing most of this paper's improvements, but it is not adequate for seeing the speedup from GMP to MPFQ.

10.2. Impact of $B_{1}$ and $d_{1}$ for 20-bit primes. Recall from Section9 that EECMMPFQ's first curve finds 12467 of the 38635 20-bit primes using $B_{1}=256$ and $d_{1}=1$. This experiment used a total of $65900078 \mathrm{M}+55479860 \mathrm{~S}$; each successful prime therefore consumed 9736 modular multiplications.

We tried the same curve again using $B_{1}=37, d_{1}=90, e=1$, and $\#\{i\}=\#\{j\}$. This time EECM-MPFQ found 1527 primes in stage 1 and an additional 14017 primes in stage 2 (1242 during conversion to affine and 12775 at the end of stage 2 ), for an overall success probability of $15544 / 38635 \approx 40.2329 \%$. The cost of handling a worst-case input was $734 \mathbf{M}+212 \mathbf{S}$, and if EECM-MPFQ had incurred 


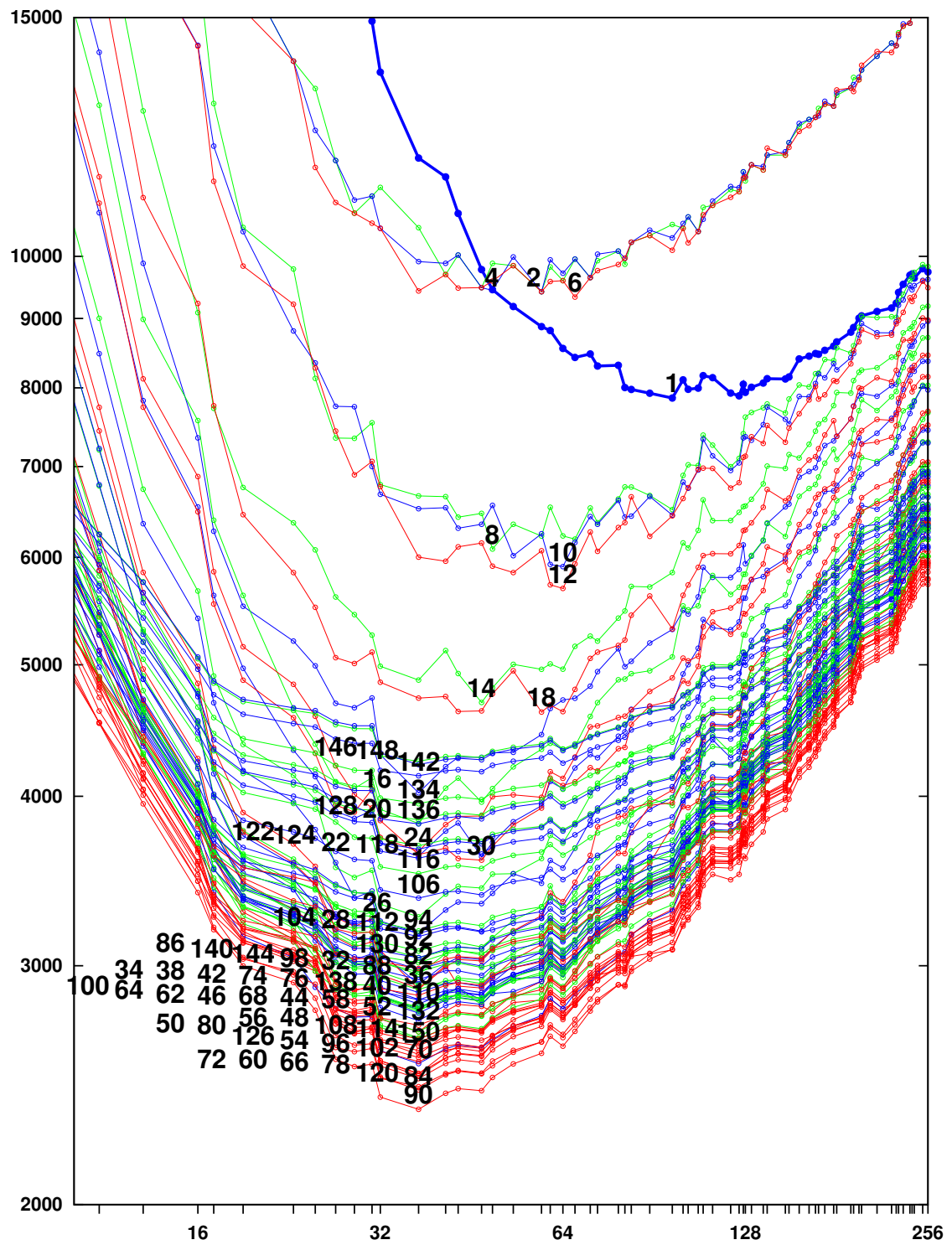

Figure 10.1. Cost ratio for the curve $x^{2}+y^{2}=1-(24167 / 25) x^{2} y^{2}$ with torsion group $\mathbf{Z} / 12 \mathbf{Z}$ and base point $(5 / 23,-1 / 7)$. The vertical axis is the number of modular multiplications used for both stage 1 and stage 2, times the number of primes in $\left[2^{19}, 2^{20}\right]$, divided by the number of primes found. The horizontal axis is $B_{1}$. The label inside the graph is $d_{1}$. Data points with the same $d_{1}$ are connected by line segments. 
TABLE 10.1. Cost ratio for sample sets of $b$-bit primes for $b \in\{15,16, \ldots, 50\}$. "Samples" is the size of the sample set. "Pr" is the success probability, within the sample set, of the curve $x^{2}+y^{2}=1-(24167 / 25) x^{2} y^{2}$ with base point $(5 / 23,-1 / 7)$ and torsion group $\mathbf{Z} / 12 \mathbf{Z}$, using EECM-MPFQ parameters $B_{1}, d_{1}$, $e$, and $\#\{i\} / \#\{j\}=R$. "Mults" is the number of modular multiplications used for both stage 1 and stage 2. "Ratio" is "mults" divided by "Pr"; i.e., the number of modular multiplications per prime found. The logarithm of "ratio" is "power" times $\sqrt{2 \log 2^{b} \log \log 2^{b}}$. "Savings" is the fraction of modular multiplications saved within the sample set by primes found before the end of stage 2. "Cycles" is the number of cycles used for both stage 1 and stage 2 on a $3.2 \mathrm{GHz}$ AMD Phenom II X4 (100f42) for $n$ between 192 bits and 256 bits, divided by "Pr"; i.e., the number of cycles per prime found.

\begin{tabular}{|c|c|c|c|c|c|c|c|c|c|c|c|}
\hline its & $B_{1}$ & $d_{1}$ & & $\bar{R}$ & samples & $\operatorname{Pr}$ & mults & ratio & power & savings & cycles \\
\hline 15 & 16 & 60 & & 1 & 1612 & $67 \%$ & 475 & 725.8 & 0.9440 & $6.0743 \%$ & 398383 \\
\hline 16 & 16 & 60 & & 1 & 3030 & $41 \%$ & 475 & 938.8 & & & \\
\hline 17 & 27 & 60 & & 1 & 5709 & $6 \%$ & 632 & 1155.7 & & & 2882 \\
\hline 18 & 27 & 90 & & 1 & 749 & & 816 & .3 & & & 58 \\
\hline 19 & 37 & 90 & 1 & 1 & 390 & $8 \%$ & 946 & & & & \\
\hline 20 & 37 & 90 & 1 & 1 & 35 & & 9 & & & & 22 \\
\hline 21 & 37 & 90 & 1 & 1 & 36 & & & & & & 364 \\
\hline 22 & 47 & 120 & 1 & 1 & 336 & & 92 & & & & $10 ?$ \\
\hline 23 & 64 & 20 & 1 & 1 & & & & & & & 28 \\
\hline 24 & 81 & 0 & 1 & 1 & & & 76 & & & & 37. \\
\hline 5 & 97 & 0 & 1 & 1 & & & 2427 & & & & 59 \\
\hline 26 & 97 & 0 & 1 & 1 & & & & & & & \\
\hline 27 & 13 & 0 & 1 & 1 & & & 2 & & & & \\
\hline 28 & 13 & 0 & 1 & 1 & & & 29 & & & & \\
\hline 29 & 14 & 0 & 1 & 1 & & & 30 & & & & \\
\hline 30 & 14 & 0 & 1 & 1 & & & 3065 & & & & 323 \\
\hline 31 & 263 & 10 & 1 & 2 & & & 76 & & & & \\
\hline 32 & 263 & 10 & 1 & 2 & & & 5376 & & & & \\
\hline 33 & 26 & 210 & 1 & 2 & & & 5376 & & & & 578 \\
\hline 34 & 3 & 30 & 1 & 1 & & & & & & & \\
\hline 35 & 389 & 420 & 1 & 1 & & & & & & & \\
\hline 36 & 433 & 420 & 1 & 1 & & & & & & & \\
\hline 37 & 521 & 420 & 1 & 1 & & & 9909 & & & & \\
\hline $3 \varepsilon$ & 521 & 420 & 1 & 1 & & & 09 & & & & 961 \\
\hline 3 & 587 & 420 & 1 & 1 & & & 621 & 0.3 & & & $71 \xi$ \\
\hline 40 & 587 & 420 & 1 & 1 & 8576 & & 621 & 2.8 & & & 13 \\
\hline 41 & 9 & 330 & 1 & 1 & 20. & & 36 & .5 & & & 611337 \\
\hline 42 & 1 & 630 & & 1 & & & 36 & & & & 74 \\
\hline 4 & & 630 & 1 & 1 & & & & & & & \\
\hline 4 & & 630 & 1 & 1 & & & & & & & \\
\hline 4 & & 30 & 1 & 1 & & & 208 & & & & \\
\hline 46 & & 630 & 1 & 1 & & & 22884 & & & & \\
\hline 47 & & 40 & 1 & 1 & & & 32129 & & & & \\
\hline $4 \varepsilon$ & & 840 & & 1 & & & & & & & \\
\hline $4 !$ & & 840 & & 1 & & & & & & & 42 \\
\hline 50 & 521 & 840 & 2 & 1 & 1048576 & $5 \%$ & 42981 & 546.8 & 0.8971 & $0.1686 \%$ & 29654218 \\
\hline
\end{tabular}


this cost for every input then it would have used a total of $28358090 \mathrm{M}+8190620 \mathrm{~S}$, i.e., just 2351 modular multiplications per successful prime. EECM-MPFQ actually used only $27357827 \mathrm{M}+8172296 \mathrm{~S}$, saving $2.8 \%$, because primes found in stage 1 did not incur the costs of stage 2 .

Figure 10.1 shows the results of similar computations for many more pairs $\left(B_{1}, d_{1}\right)$. The figure quantifies the well-known importance of stage $2: \quad d_{1}=1$ costs more than three times as many modular multiplications as the best $d_{1}$ for 20 -bit primes. The figure also confirms the idea that $d_{1}$ should have several small prime factors. Each computation used $e=1$ and used EECM-MPFQ's default ratio $\#\{i\} / \#\{j\}=1$.

10.3. Other sizes of primes. Table 10.1 reports the effectiveness of good choices of $\left(B_{1}, d_{1}, e, \#\{i\} / \#\{j\}\right)$ for 15 -bit primes, 16-bit primes, 17-bit primes, and so on through 50-bit primes. The "power" column shows that EECM-MPFQ uses fewer than $\exp \left(0.9 \sqrt{2 \log 2^{b} \log \log 2^{b}}\right)$ modular multiplications per $b$-bit prime found, for each $b \in\{25,26, \ldots, 50\}$. See http://eecm.cr.yp.to for performance data for larger values of $b$.

The conventional wisdom (see, e.g., [18) is that one should use Pollard's rho method for primes up to about 30 bits and then switch over to ECM. We present ECM performance data for much smaller sizes as a basis for comparison and for future improvements. Our guess is that improvements in ECM have drastically reduced the optimal rho-to-ECM cutoff.

\section{ACKNOWLEDGMENTS}

The authors wish to thank Paul Zimmermann for many detailed comments and suggestions; Igor Shparlinski for his challenging questions regarding ECM effectiveness and optimization, prompting the studies reported in Sections 9 and 10 of this paper; and the anonymous referees for their suggestions.

\section{REFERENCES}

[1] SPEED: software performance enhancement for encryption and decryption, 2007. http:// www.hyperelliptic.org/SPEED. See [27].

[2] Michel Abdalla, Paulo S. L. M. Barreto (editors), Progress in cryptology-LATINCRYPT 2010, first international conference on cryptology and information security in Latin America, Puebla, Mexico, August 8-11, 2010, proceedings, Lecture Notes in Computer Science, 6212, Springer, 2010. See [9].

[3] A. O. L. Atkin, Francois Morain, Finding suitable curves for the elliptic curve method of factorization, Mathematics of Computation 60 (1993), 399-405. ISSN 0025-5718. http://www . lix.polytechnique.fr/ morain/Articles/articles.english.html. Citations in this document: $\S 1.2, \S 7, \S 7.1$. MR $93 \mathrm{k}: 11115$

[4] Michael A. Bennett, Bruce C. Berndt, Nigel Boston, Harold G. Diamond, Adolf J. Hildebrand, Walter Philipp (editors), Number theory for the millennium. I: papers from the conference held at the University of Illinois at Urbana-Champaign, Urbana, IL, May 21-26, 2000, A. K. Peters, Natick, Massachusetts, 2002. ISBN 1-56881-126-8. MR2003h:11004 See [5].

[5] Daniel J. Bernstein, Arbitrarily tight bounds on the distribution of smooth integers, in Number Theory 2000 [4] (2002), 49-66. http://cr.yp.to/papers.html\#psi. Citations in this document: §9.4. MR 1956218 (2004b:11135)

[6] Daniel J. Bernstein, Scaled remainder trees (2004). http://cr.yp.to/papers.html\# scaledmod. Citations in this document: $§ 5.3$.

[7] Daniel J. Bernstein, Fast multiplication and its applications, in Algorithmic Number Theory [19] (2008), 325-384. http://cr.yp.to/papers.html\#multapps. Citations in this document: §5.3. MR2467550(2010a:68186) 
[8] Daniel J. Bernstein, Peter Birkner, Marc Joye, Tanja Lange, Christiane Peters, Twisted Edwards curves, in Africacrypt 2008 [45] (2008), 389-405. http://eprint.iacr.org/2008/ 013. Citations in this document: $\S 2.2, \S 2.4, \S 2.5, \S 3, \S 7$. MR2482341 (2010e:11057)

[9] Daniel J. Bernstein, Peter Birkner, Tanja Lange, Starfish on strike, in Latincrypt 2010, [2] (2010), 62-80. http://eprint.iacr.org/2010/367. Citations in this document: $\S 1.2$.

[10] Daniel J. Bernstein, Peter Birkner, Tanja Lange, Christiane Peters, Optimizing double-base elliptic-curve single-scalar multiplication, in Indocrypt 2007 [42] (2007), 167-182. http:// eprint.iacr.org/2007/414. Citations in this document: §1.1. MR2570254 (2010k:94034)

[11] Daniel J. Bernstein, Tien-Ren Chen, Chen-Mou Cheng, Tanja Lange, Bo-Yin Yang, ECM on graphics cards, in Eurocrypt 2009 [30] (2009), 483-501. http://eprint.iacr.org/2008/480. Citations in this document: $\S 1$. MR2538444

[12] Daniel J. Bernstein, Tanja Lange, Explicit-formulas database (2007). http://hyperelliptic. org/EFD. Citations in this document: $\S 2$.

[13] Daniel J. Bernstein, Tanja Lange, Faster addition and doubling on elliptic curves, in Asiacrypt 2007 [32] (2007), 29-50. http://eprint.iacr.org/2007/286. Citations in this document: $\S 1.1, \S 2.2, \S 2.4, \S 2.9$. MR2565722(2011d:11125)

[14] Daniel J. Bernstein, Tanja Lange, Inverted Edwards coordinates, in AAECC 2007 [17] (2007), 20-27. http://eprint.iacr.org/2007/410. Citations in this document: $\S 1.1, \S 2.5$.

[15] Daniel J. Bernstein, Tanja Lange, Analysis and optimization of elliptic-curve single-scalar multiplication, in Fq8 [38] (2008), 1-19. http://eprint.iacr.org/2007/455. Citations in this document: §1.1, §4.5. MR2436321 (2010a:94049)

[16] Daniel J. Bernstein, Tanja Lange, A complete set of addition laws for incomplete Edwards curves, Journal of Number Theory, 131, 858-872. http://eprint.iacr.org/2009/580. Citations in this document: $\S 2.9, \S 2.9, \S 2.9, \S 3$. MR 2772476

[17] Serdar Boztas, Hsiao-Feng Lu, Applied algebra, algebraic algorithms and error-correcting codes, 17th international symposium, AAECC-17, Bangalore, India, December 16-20, 2007, proceedings, Lecture Notes in Computer Science, 4851, Springer, 2007. ISBN 978-3-54077223-1. See [14]. MR2640522 (2011a:94003)

[18] Richard P. Brent, Some integer factorization algorithms using elliptic curves, Australian Computer Science Communications 8 (1986), 149-163. ISSN 0157-3055. http://maths . anu . edu.au/ brent/pub/pub102.html. Citations in this document: §4.2, §4.3, §9.3, §9.4, §10.3. See [44].

[19] Joe Buhler, Peter Stevenhagen, Algorithmic number theory: lattices, number fields, curves and cryptography, Mathematical Sciences Research Institute Publications, 44, Cambridge University Press, 2008. ISBN 978-0521808545. MR2009h:11003 See [7].

[20] David V. Chudnovsky, Gregory V. Chudnovsky, Sequences of numbers generated by addition in formal groups and new primality and factorization tests, Advances in Applied Mathematics 7 (1986), 385-434. MR88h:11094 Citations in this document: $\S 4.4, \S 4.4, \S 4.7$.

[21] Romain Cosset, Factorization with genus 2 curves, Mathematics of Computation 79 (2010), 1191-1208. http://arxiv.org/pdf/0905.2325. Citations in this document: §4.7, §4.7, §4.7, §4.7. MR2600562(2011d:11289)

[22] Peter de Rooij, Efficient exponentiation using precomputation and vector addition chains, in Eurocrypt 1994 [23] (1995), 389-399. MR1479665 Citations in this document: §5.6.

[23] Alfredo De Santis (editor), Advances in cryptology-EUROCRYPT '94, workshop on the theory and application of cryptographic techniques, Perugia, Italy, May 9-12, 1994, proceedings, Lecture Notes in Computer Science, 950, Springer, Berlin, 1995. ISBN 3-540-60176-7. MR:98h:94001 See [22].

[24] Karl Dickman, On the frequency of numbers containing primes of a certain relative magnitude, Arkiv för Matematik, Astronomi och Fysik 22 (1930), 1-14. ISSN 0365-4133. Citations in this document: $§ 9.3$.

[25] Harold M. Edwards, A normal form for elliptic curves, Bulletin of the American Mathematical Society 44 (2007), 393-422. http://www.ams.org/bull/2007-44-03/ S0273-0979-07-01153-6/home.html. Citations in this document: $§ 2.2$. MR2318157 (2008b:14052)

[26] Pierrick Gaudry, Fast genus 2 arithmetic based on theta functions, Journal of Mathematical Cryptology 1 (2007), 243-265. http://www.loria.fr/ gaudry/publis/arithKsurf.pdf. Citations in this document: $\S 4.7, \S 4.7$. MR2372155 (2009f:11156) 
[27] Pierrick Gaudry, Emmanuel Thomé, The mpFq library and implementing curve-based key exchanges, in [1] (2007), 49-64. http://www.loria.fr/ gaudry/papers.en.html. Citations in this document: $\S 4.6$.

[28] Florian Hess, Sebastian Pauli, Michael E. Pohst (editors), Algorithmic number theory, proceedings of the 7th international symposium (ANTS-VII) held at the Technische Universität Berlin, Berlin, July 23-28, 2006, Lecture Notes in Computer Science, 4076, Springer, Berlin, 2006. ISBN 3-540-36075-1. MR2007h:11001 See [47].

[29] Huseyin Hisil, Kenneth Koon-Ho Wong, Gary Carter, Ed Dawson, Twisted Edwards curves revisited, in Asiacrypt 2008 [39] (2008). http://eprint.iacr.org/2008/522. Citations in this document: $\S 1.1, \S 2.3, \S 2.6, \S 2.6$. MR 2546103

[30] Antoine Joux (editor), Advances in cryptology-EUROCRYPT 2009, 28th annual international conference on the theory and applications of cryptographic techniques, Cologne, Germany, April 26-30, 2009, proceedings, Lecture Notes in Computer Science, 5479, Springer, 2009. ISBN 978-3-642-01000-2. See [11]. MR2590599 (2010j:94007)

[31] Alexander Kruppa, Améliorations de la multiplication et de la factorisation d'entier, Ph.D. thesis, Université Henri Poincaré Nancy I, 2010. http://tel.archives-ouvertes.fr/ tel-00477005/en/. Citations in this document: $\S 7$.

[32] Kaoru Kurosawa (editor), Advances in cryptology-ASIACRYPT 2007, 13th international conference on the theory and application of cryptology and information security, Kuching, Malaysia, December 2-6, 2007, proceedings, Lecture Notes in Computer Science, 4833, Springer, 2007. ISBN 978-3-540-76899-9. See [13]. MR 2590581(2010i:94001)

[33] Hendrik W. Lenstra, Jr., Factoring integers with elliptic curves, Annals of Mathematics 126 (1987), 649-673. ISSN 0003-486X. MR89g:11125. https://openaccess.leidenuniv.nl/ bitstream/1887/3826/1/346_086.pdf. Citations in this document: $\S 1, \S 9.4$.

[34] Barry Mazur, Rational isogenies of prime degree, Inventiones Mathematicae 4 (1978), 129162. Citations in this document: $\S 6$. MR 482230 (80h:14022)

[35] James McKee, Subtleties in the distribution of the numbers of points on elliptic curves over a finite prime field, Journal of the London Mathematical Society 59 (1999), 448-460. Citations in this document: $§ 4.1, \S 9.4$. MR 1709178 (2000g:11055)

[36] Peter L. Montgomery, Speeding the Pollard and elliptic curve methods of factorization, Mathematics of Computation 48 (1987), 243-264. ISSN 0025-5718. MR88e:11130 http://www . ams .org/mcom/1987-48-177/S0025-5718-1987-0866113-7/S0025-5718-1987-0866113-7. pdf. Citations in this document $\S 4.3, \S 4.4, \S 7, \S 7.7$. See [44].

[37] Peter L. Montgomery, An FFT extension of the elliptic curve method of factorization, Ph.D. thesis, University of California at Los Angeles, 1992. ftp://ftp.cwi.nl/pub/pmontgom/ ucladissertation.psl.gz. Citations in this document: §5.4, §5.4, §7, §7, §9.3, §10.1. MR2688742

[38] Gary L. Mullen, Daniel Panario, Igor E. Shparlinski (editors), Finite fields and applications: papers from the 8th international conference held in Melbourne, July 9-13, 2007, Contemporary Mathematics, 461, American Mathematical Society, 2008. ISBN 978-0-8218-4309-3. MR2009h:11004 See [15].

[39] Josef Pieprzyk (editor), Advances in cryptology-ASIACRYPT 2008, 14th international conference on the theory and application of cryptology and information security, Melbourne, Australia, December 7-11, 2008, Lecture Notes in Computer Science, 5350, 2008. ISBN 9783-540-89254-0. See [29]. MR2590580 (2010j:94005)

[40] John M. Pollard, Theorems on factorization and primality testing, Proceedings of the Cambridge Philosophical Society 76 (1974), 521-528. ISBN 0305-0041. MR50:6992. http://cr.yp.to/bib/entries.html\#1974/pollard. Citations in this document: $₫ 4.2, \S 4.3$.

[41] Robert D. Silverman, Samuel S. Wagstaff, Jr., A practical analysis of the elliptic curve factoring algorithm, Mathematics of Computation 61 (1993), 445-462. http: //www . ams .org/ journals/mcom/1993-61-203/S0025-5718-1993-1122078-7/S0025-5718-1993-1122078-7. pdf. Citations in this document: $§ 9.3$. MR1122078 (93k:11117)

[42] Kannan Srinathan, Chandrasekaran Pandu Rangan, Moti Yung (editors), Progress in cryptology-INDOCRYPT 2007, 8th international conference on cryptology in India, Chennai, India, December 9-13, 2007, proceedings, Lecture Notes in Computer Science, 4859, Springer, 2007. ISBN 978-3-540-77025-1. See [10]. MR2574217(2010g:94137)

[43] William Stein (editor), Sage Mathematics Software (Version 3.2.3), The Sage Group, 2009. http://www.sagemath.org. Citations in this document: $\S 6.10$. 
[44] Hiromi Suyama, Informal preliminary report (8), cited in [18] as personal communication and in [36] (1985). Citations in this document: $\S 7$.

[45] Serge Vaudenay, Progress in cryptology - AFRICACRYPT 2008, First international conference on cryptology in Africa, Casablanca, Morocco, June 11-14, 2008, proceedings, Lecture Notes in Computer Science, 5023, Springer, 2008. ISBN 978-3-540-68159-5. See [8]. MR:2528441(2009m:94064)

[46] Paul Zimmermann, 50 largest factors found by ECM. http://www.loria.fr/ zimmerma/ records/top50.html. Citations in this document: $\S 1$.

[47] Paul Zimmermann, Bruce Dodson, 20 years of ECM, in ANTS VII [28] (2006), 525542. http://www.loria.fr/ zimmerma/papers/40760525.pdf. Citations in this document: 11 , $\S 4.2, \S 4.3, \S 4.4, \S 4.5, \S 5.3, \S 7 . \mathrm{MR} 2282947(2007 \mathrm{j}: 11172)$

Department of Computer Science (MC 152), University of Illinois at Chicago, Chichgo, Illinois 60607-7053

E-mail address: djb@cr.yp.to

Department of Mathematics and Computer Science, Technische Universiteit Eindhoven, P.O. Box 513, 5600 MB Eindhoven, Netherlands

E-mail address: pbirkner@fastmail.fm

Department of Mathematics and Computer Science, Technische Universiteit EindHoven, P.O. Box 513, 5600 MB Eindhoven, Netherlands

E-mail address: tanja@hyperelliptic.org

Department of Mathematics, Technical University of Denmark, 2800 Kgs. Lyngby, DENMARK

E-mail address: c.p.peters@mat.dtu.dk 